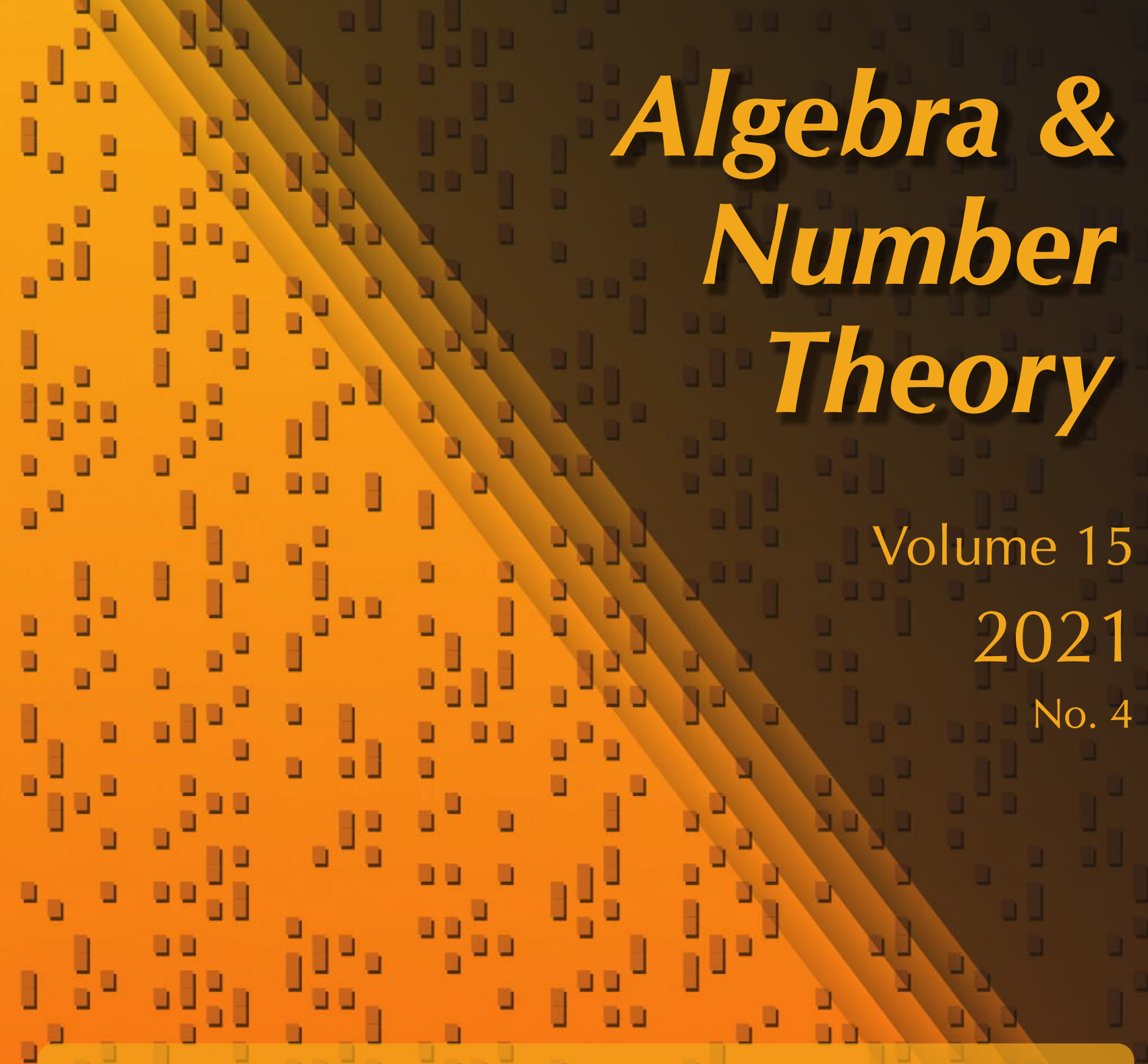

On the $\mu$-invariants of abelian varieties over function fields of positive characteristic

King-Fai Lai, Ignazio Longhi, Takashi Suzuki, Ki-Seng Tan and Fabien Trihan

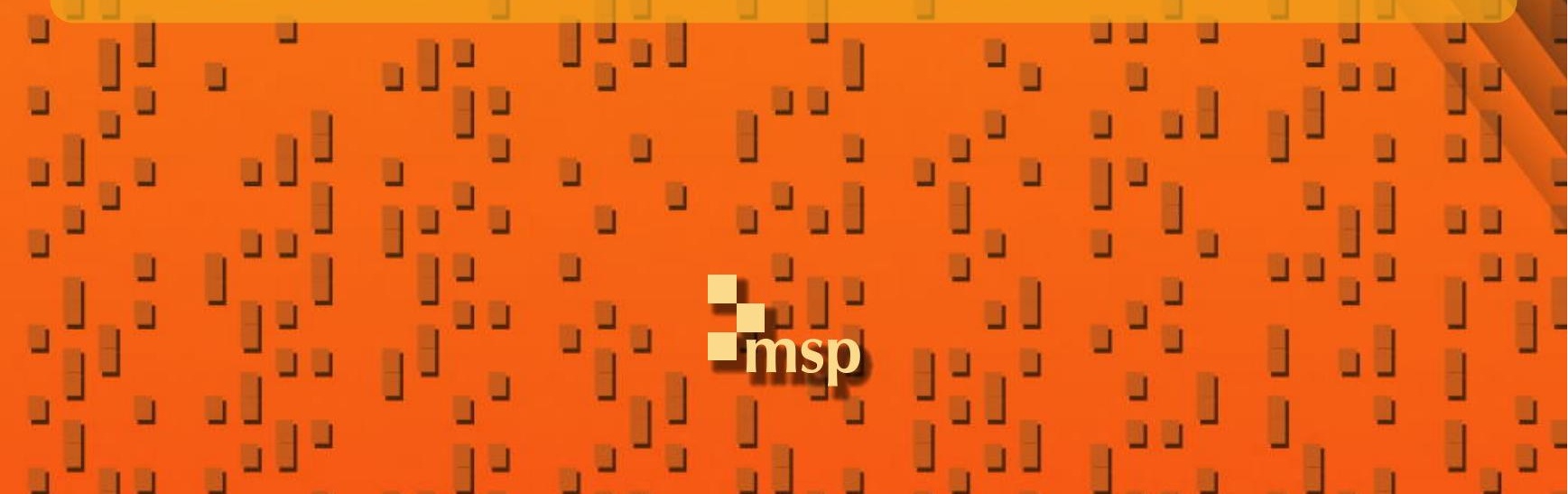




\title{
On the $\mu$-invariants of abelian varieties over function fields of positive characteristic
}

\author{
King-Fai Lai, Ignazio Longhi, Takashi Suzuki, Ki-Seng Tan and Fabien Trihan
}

Let $A$ be an abelian variety over a global function field $K$ of characteristic $p$. We study the $\mu$-invariant appearing in the Iwasawa theory of $A$ over the unramified $\mathbb{Z}_{p}$-extension of $K$. Ulmer suggests that this invariant is equal to what he calls the dimension of the Tate-Shafarevich group of $A$ and that it is indeed the dimension of some canonically defined group scheme. Our first result is to verify his suggestions. He also gives a formula for the dimension of the Tate-Shafarevich group (which is now the $\mu$-invariant) in terms of other quantities including the Faltings height of $A$ and Frobenius slopes of the numerator of the Hasse-Weil $L$-function of $A / K$ assuming the conjectural Birch-Swinnerton-Dyer formula. Our next result is to prove this $\mu$-invariant formula unconditionally for Jacobians and for semistable abelian varieties. Finally, we show that the " $\mu=0$ " locus of the moduli of isomorphism classes of minimal elliptic surfaces endowed with a section and with fixed large enough Euler characteristic is a dense open subset.

1. Introduction $\quad 863$

2. The $\mu$-invariant for abelian varieties $\quad 869$

3. The $\mu$-invariant for Jacobians $\quad 879$

4. The $\mu$-invariant for semistable abelian varieties $\quad 886$

5. The $\mu$-invariant for elliptic curves $\quad 889$

6. $\mu=0$ for generic elliptic curves $\quad 893$

7. Computations 898

Appendix: Integrality of sum of slopes less than one $\quad 902$

$\begin{array}{ll}\text { Acknowledgments } & 904\end{array}$

$\begin{array}{ll}\text { References } & 904\end{array}$

\section{Introduction}

Let $\Gamma$ be a profinite group isomorphic to $\mathbb{Z}_{p}$ and let $\Lambda$ denote the completed group algebra $\mathbb{Z}_{p} \llbracket \Gamma \rrbracket$. By a general structure theorem, if $M$ is a finitely generated torsion $\Lambda$-module then one has a pseudoisomorphism (that is, a homomorphism with finite kernel and cokernel)

$$
M \sim \bigoplus_{i} \Lambda /\left(p^{m_{i}}\right) \oplus \bigoplus_{j} \Lambda /\left(f_{j}\right),
$$

Suzuki is a Research Fellow of Japan Society for the Promotion of Science and supported by JSPS KAKENHI Grant Number JP18J00415. Tan was supported in part by the Ministry of Science and Technology of Taiwan, MOST 107-2115-M-002-010-MY2. It is our pleasure to thank NCTS/TPE for supporting a number of meetings of the authors in National Taiwan University.

MSC2010: primary 11R23; secondary 11G10, 11S40, $14 \mathrm{~J} 27$.

Keywords: Iwasawa theory, abelian variety, Selmer group, $\mu$-invariant, elliptic surface. 
where the elements $f_{j}$ can be identified with certain irreducible polynomials after choosing a topological generator of $\Gamma$ and hence fixing an isomorphism between $\Lambda$ and the power series ring $\mathbb{Z}_{p} \llbracket t \rrbracket$. In particular (1) makes it possible to define the number

$$
\mu(M):=\sum_{i} m_{i},
$$

which is called the $\mu$-invariant of $M$. The algebraic side of Iwasawa theory deals with modules over rings exemplified by $\Lambda$ and understanding their $\mu$-invariant has always been an interesting question. For example, in the setting above $\mu(M)=0$ means that $M$ is finitely generated as a $\mathbb{Z}_{p}$-module. More generally, let $M_{\Gamma p^{n}}$ denote the set of coinvariants for the subgroup of index $p^{n}$. Then (1) implies the formula

$$
\frac{\log \left|M_{\Gamma p^{n}}\right|}{p^{n} \log p}=\mu(M)+o(1) \quad \text { as } n \rightarrow \infty,
$$

provided that $\left|M_{\Gamma^{p^{n}}}\right|<\infty$ holds for every $n$.

The $\mu$-invariant was first studied in the following situation. Let $F$ be a number field and let $F^{\text {cyc }}$ denote its $\mathbb{Z}_{p}$-cyclotomic extension: then one takes $\operatorname{Gal}\left(F^{\mathrm{cyc}} / F\right)$ as $\Gamma$ and $\mathbb{Z}_{p} \llbracket \mathrm{Gal}\left(F^{\mathrm{cyc}} / F\right) \rrbracket$ as $\Lambda$. The $\Lambda$-module to be considered is $M\left(F^{\mathrm{cyc}} / F\right)$, the inverse limit of the $p$-parts of class groups in the intermediate extensions of $F^{\mathrm{cyc}} / F$. Iwasawa showed that $M\left(F^{\mathrm{cyc}} / F\right)$ is finitely generated and torsion and he conjectured the equality $\mu\left(M\left(F^{\mathrm{cyc}} / F\right)\right)=0$. His conjecture was proved by Ferrero-Washington in the case when $F / \mathbb{Q}$ is an abelian extension, but it is still open in the general setting. On the other hand, Iwasawa [1973] also gave examples of $\mathbb{Z}_{p}$-extensions $L / F$ of number fields such that $\mu(M(L / F))>0$.

The $\Lambda$-modules of interest in this paper are duals of Selmer groups of abelian varieties over function fields. The Iwasawa theory of abelian varieties was started by Mazur [1972], where he already gave some examples of elliptic curves with nonvanishing $\mu$ over $\mathbb{Q}^{\text {cyc }}$ [Mazur 1972, §10]. More recently Coates and Sujatha [2005, Conjecture A] have conjectured that the $\mu$-invariant of an elliptic curve always vanishes in the cyclotomic extension of a number field if one replaces the Selmer group with the fine Selmer group; see also [Sujatha 2010; 2011] for an introduction to these ideas.

1.1. Our setting: $\mathbb{Z}_{p}$-extensions of function fields. In the following, $K$ is a function field in one variable over a finite field of characteristic $p$. The $\mathbb{Z}_{p}$-extension of $K$ we are going to consider is the arithmetic one, that is, the unique $\mathbb{Z}_{p}$-extension obtained by enlarging the field of constants $\mathbb{F}_{q}$, which is an obvious analogue of $F^{\mathrm{cyc}} / F$.

1.1.1. $\mu$-invariants over function fields: class groups. In this paper, we study the $\mu$-invariant attached to abelian varieties. Before discussing it, we review the situation for class groups, to place our results better in context. Let $h_{n}$ denote the cardinality of the $p$-part of the class group at the $n$-th layer of the extension. In the case of the arithmetic $\mathbb{Z}_{p}$-extension, the growth of $h_{n}$ can be computed from the zeta function and Weil's work on the Riemann hypothesis gives some estimation. Interpreting class groups as sets of rational points in a Jacobian, geometric considerations and some simple Galois theory yield a more precise result: see [Rosen 1973]. The outcome is that $\log \left(h_{n}\right)$ is proportional to $n$, for $n$ large enough: 
that is, the relevant $\mu$-invariant vanishes [Li and Zhao 1997, Proposition 5.4]. (It might be worthwhile to recall that similar observations and the attempt to develop an analogue theory for number fields were Iwasawa's starting point: see [Thakur 1994] for more on this function field-number field dialogue.)

Things change rather dramatically if instead one takes a geometric $\mathbb{Z}_{p}$-extension of $K-$ that is, an extension where the constant field is the same at any layer. Such extensions can ramify at infinitely many different places and in such a situation the limit of class groups is not a noetherian $\Lambda$-module [Gold and Kisilevsky 1988, Theorem 2]. Moreover, it turns out that in a geometric $\mathbb{Z}_{p}$-extension $\log \left(h_{n}\right)$ grows at least as $p^{2 n}$ [Gold and Kisilevsky 1988, Theorem 1].

Remark 1.1.2. In the function field setting there is also a different kind of "cyclotomic" extension, obtained by adding to $K$ the $\mathfrak{p}^{\infty}$-torsion of a rank 1 Drinfeld module, for $\mathfrak{p}$ a place of $K$. It turns out that when $K$ is the rational function field (that is, $K=\mathbb{F}_{q}(\theta)$ ) and the cyclotomic extension is generated through the Carlitz module, an analogue of the Ferrero-Washington theorem holds [Anglès et al. 2020, Theorem 1.3]. An important difference with the situation we discussed before is that the Galois group of the extension in [Anglès et al. 2020] is isomorphic to an infinite product of copies of $\mathbb{Z}_{p}$ : hence the corresponding Iwasawa algebra is not even noetherian. A general theory for modules over such algebras is still lacking, so it is not completely clear what is the meaning (or the general definition) of a $\mu$-invariant in this setting. The result in [Anglès et al. 2020] consists in proving that a certain $p$-adic $L$-function generates the ( $\chi$-part of the) Fitting ideal of a limit of class groups, and computing that the reduction of this $L$-function modulo $p$ is not zero.

1.1.3. $\mu$-invariants over function fields: abelian varieties. For an abelian variety $A / K$, Iwasawa theory over the arithmetic extension of $K$ was first developed in [Ochiai and Trihan 2009]. In particular, [loc. cit., Theorem 1.7] proves that the dual of the Selmer group is a finitely generated torsion $\Lambda$-module: thus one can apply (1) and define a $\mu$-invariant $\mu_{A / K}$. Moreover, [loc. cit., Theorem 1.8] gives some conditions for its vanishing when $A$ is isotrivial: more precisely,

- $\mu_{A / K}=0$ always holds if, after base change by a finite extension, $A$ becomes isomorphic to a constant ordinary abelian variety;

- in the supersingular case, $\mu_{A / K}=0$ is true if $A$ becomes constant over a finite extension of $K$ with invertible Hasse-Witt matrix. ${ }^{1}$

Finally, [loc. cit., Theorem 1.9] shows an application of $\mu_{A / K}=0$ to noncommutative Iwasawa theory.

We are not aware of other works discussing the $\mu$-invariants of abelian varieties over global function fields, except the paper of Ulmer that we will discuss below.

1.1.4. Ulmer's notion of the dimension of $\amalg$. Outside the context of Iwasawa theory, Ulmer [2019, Section 4] defines a certain number called the dimension of $\amalg(A)$ and denoted as $\operatorname{dim} \amalg(A)$, by looking at the asymptotic behavior of the order of the $p$-primary part of the Tate-Shafarevich group $\amalg(A)$ of an

\footnotetext{
${ }^{1}$ In [Ochiai and Trihan 2009, Theorem 1.8], this is given as an "if and only if". However, that is wrong: we discuss it in Section 7.5.
} 
abelian variety $A / K$ under extensions of the constant field $\mathbb{F}_{q}$, assuming the finiteness of Tate-Shafarevich groups. His aim is to study the so-called Brauer-Siegel problem for abelian varieties over function fields using $\operatorname{dim} \amalg(A)$. The relevance of his work to our context of Iwasawa theory lies in his suggestion [Ulmer 2019, Remark 4.3(5)] of the potential relation between $\operatorname{dim} \amalg(A)$ and the $\mu$-invariant $\mu_{A / K}$.

By the result of Kato and Trihan [2003], the assumption of the finiteness of Tate-Shafarevich groups implies the Birch and Swinnerton-Dyer formula for the leading coefficient of the Hasse-Weil $L$-function of $A / K$ at $s=1$. Using this formula, Ulmer [2019, Proposition 4.2] gives a formula for $\operatorname{dim} \amalg(A)$ in terms of various other quantities including the degree of the Hodge bundle of the Nerron model of $A$ (the function field analogue of the Faltings height) and slopes ( $p$-adic valuations of the roots) of the numerator of the $L$-function of $A / K$. From this formula, he deduces that the limit defining $\operatorname{dim} \amalg(A)$ exists (as a real number a priori!) and is an integer. However, there is a gap in the proof of this final integrality result, since the corresponding integrality property of the quantity involving slopes is not properly justified there. It only shows that $e \cdot \operatorname{dim} \amalg(A)$ is an integer, where $q=p^{e}$. We will see (and solve) this problem in the Appendix of this paper.

Ulmer [2019, Remark 4.3(2)] justifies the terminology "dimension of W" in the special case where $A / K$ is the Jacobian of a proper smooth curve over $K$. In this case, its proper flat regular model gives a surface $\mathcal{S}$ over $\mathbb{F}_{q}$. He relates the definition of $\operatorname{dim} \amalg(A)$ to the genuine dimension of the actual group scheme $\underline{\mathrm{H}}^{2}\left(p^{\infty}\right)$ over $\mathbb{F}_{q}$ defined by Artin [1974b] and Milne [1976], which is the canonical group scheme structure on the second flat cohomology of $\mathcal{S}$. Ulmer [2019, Remark 4.3(3,4)] then suggests a relation between his formula for $\operatorname{dim} \amalg(A)$ for the Jacobian $A / K$ and Milne's formula [1975, the last equation of Section 6] on Euler characteristics for the surface $\mathcal{S}$, still assuming the finiteness of Tate-Shafarevich groups.

This relation between Ulmer's work and Artin and Milne's work does not easily generalize to arbitrary abelian varieties over $K$, since an arbitrary abelian variety is not necessarily a product factor but only an isogeny factor of a Jacobian, and the degree of the needed isogeny might be a power of $p$ that kills the (unipotent) identity component of $\underline{\mathrm{H}}^{2}\left(p^{\infty}\right)$.

By [Grothendieck 1968], the finiteness of $\amalg(A)$ for the Jacobian $A$ is equivalent to the finiteness of the Brauer group of $\mathcal{S}$, the latter of which is the Tate conjecture for divisors on $\mathcal{S}$. This conjecture is verified for several surfaces including the ones treated in [Ulmer 2019]. In general, it is a hard conjecture of motivic origin.

Notice that the group scheme $\underline{\mathrm{H}}^{2}\left(p^{\infty}\right)$ for a surface $\mathcal{S}$ and the $\mu$-invariant $\mu_{A / K}$ for an arbitrary abelian variety $A$ are unconditionally defined. The general philosophy here is that in Iwasawa theory and other purely $p$-adic theories, we can do much more "without hard motivic conjectures" (at least in the function field case).

1.2. Our results: contents of this paper. We assume that the field of constants of our $K$ contains $q$ elements. The same $q$ appears in our definition of the $\mu$-invariant $\mu_{A / K}$, which is slightly different from the usual one (see Definition 2.0.1). In particular, it is not a priori obvious that $\mu_{A / K}$ is always an integer: this will be a consequence of Theorem 2.4.3. 
First, we study the behavior with respect to base change (Section 2.1). We check that, if $L / K$ is a finite Galois extension, we have $\mu_{A / K} \leqslant \mu_{A / L}$ (Lemma 2.1.2) and we give conditions ensuring that $\mu_{A / K}=0$ implies $\mu_{A / L}=0$ : in particular, we prove that this implication holds in a $p$-extension unramified outside of ordinary places (Theorem 2.1.5).

In Section 2.3, we observe that $\mu_{A / K}$ is determined by the Tate-Shafarevich group of $A$ over the unramified $\mathbb{Z}_{p}$-extension. As a consequence, one can compute it from the cardinality of the $p^{m}$-torsion of $\amalg$ in intermediate extensions. This is a nontrivial step: the crucial fact here is the control theorem for Selmer groups [Tan 2010, Theorem 4], which shows how to obtain information about the Selmer groups for $A$ over the intermediate extensions from the Selmer group for $A$ over the $\mathbb{Z}_{p}$-extension. Assuming that the $p$-part of $\amalg$ is always finite in our $\mathbb{Z}_{p}$-tower, we deduce the limit formula (6) for $\mu_{A / K}$ by a somewhat technical argument (coming from the difference between $\amalg$ and Sel). This shows that Ulmer's $\operatorname{dim} \amalg(A)$ is equal to $\mu_{A / K}$, as suggested in [Ulmer 2019, Remark 4.3(5)].

In Section 2.4, following [Suzuki 2020], we define an $\mathbb{F}_{q}$-group scheme $\mathcal{G}_{A}^{(1)}$ which represents the Tate-Shafarevich group $\amalg\left(A / K \overline{\mathbb{F}}_{q}\right) .^{2}$ We prove the formula

$$
\mu_{A / K}=\operatorname{dim} \mathcal{G}_{A}^{(1)}
$$

(Theorem 2.4.3). With $\operatorname{dim} \amalg(A)=\mu_{A / K}$ (assuming the finiteness of Tate-Shafarevich groups), this formula justifies the terminology "dimension of $\amalg$ " for an arbitrary abelian variety $A / K$. It also shows that $\mu_{A / K}$ (or $\operatorname{dim} \amalg(A)$ ) is an integer. Using the group scheme $\mathcal{G}_{A}^{(1)}$ (and the related group schemes $\mathcal{G}_{A}^{(i)}$ ), we prove a control theorem for $\amalg$ (Proposition 2.4.4), which is not a consequence of the control theorem for Sel mentioned earlier. This gives another more direct proof of $\mu_{A / K}=\operatorname{dim} \amalg(A)$.

In (10), we reinterpret the formula in [Ulmer 2019, Proposition 4.2] on $\operatorname{dim} \amalg(A)$ (which assumes the finiteness of Tate-Shafarevich groups and uses the BSD formula) as a formula on $\mu_{A / K}$ and hence give an upper bound (11) for $\mu_{A / K}$. Since both of the sides of the resulting $\mu$-invariant formula (10) are unconditionally defined, it is then natural to ask if the formula (10) can be proved unconditionally. We will give partial results in subsequent sections.

In Section 3, we take $A$ to be the Jacobian of a curve $\mathcal{S}_{K}$ over $K$. In this case, we define an $\mathbb{F}_{q}$-group scheme $\mathrm{Br}$ representing the Brauer group of the associated surface $\mathcal{S}$ over $\mathbb{F}_{q}$ using the Artin-Milne group scheme $\underline{\mathrm{H}}^{2}\left(p^{\infty}\right)$. We show that the group schemes $\mathcal{G}_{A}^{(1)}$ and $\underline{\mathrm{Br}}$ are isomorphic up to finite étale groups (Proposition 3.1.5). This implies that $\mu_{A / K}=\operatorname{dim} \underline{\operatorname{Br}}$. As a consequence, we can show that in this case the formula (10) is equivalent to Milne's formula [1975, the last equation of Section 6] (Corollary 3.4.1), as suggested by Ulmer [2019, Remark 4.3(3,4)]. This proves (10) independently of the finiteness of $\amalg$. As an application, in Propositions 3.4.2 and 3.4.4, we give necessary and sufficient conditions for $\mu_{A / K}=0$ and (under some additional assumptions) $\mu_{A / K}=1$. Proposition 3.4.5 provides an explicit example of an elliptic curve with $\mu$-invariant 1 .

\footnotetext{
${ }^{2}$ This representation is a bit subtle. See the first paragraph of Section 2.4 for the precise meaning. The subtleties exist but are "controllable," which is the content of the control theorem for $\amalg$, namely Proposition 2.4.4.
} 
Section 4 deals with the case of semistable abelian varieties. Theorem 4.1.1 shows that formula (10) holds with no condition on $\amalg$ also in this setting. The proof is based on the fact that in this case the Iwasawa main conjecture holds [Lai et al. 2016] and so $\mu_{A / K}$ can be computed explicitly from the ( $p$-adic) $L$-function.

Summarizing the previous three sections, the $\mu$-invariant formula (10) is true for the following three cases:

- if the Tate-Shafarevich groups are finite (by Ulmer),

- if $A$ is a Jacobian, or

- if $A$ is semistable everywhere.

In Section 5, we specialize to $A$ being an elliptic curve. As explained in Theorem 5.1.1, this gives a simplified version of (10) and (11) and hence more instances of $\mu_{A / K}>0$ (Proposition 5.1.2). In Section 5.3, we investigate the variation of the $\mu$-invariant in isogeny classes. If $p>2$, the Legendre form of the Weierstrass equation provides sufficient conditions for $\mu_{A / K}=0$ (Theorem 5.4.2).

In Section 6, we prove that, at least for $p>3$, the vanishing of $\mu_{A / K}$ is the normal behavior in the following sense: for $n>\frac{1}{2}(g-1)$ (where $g$ is the genus of the curve corresponding to $K$ ), we build an irreducible variety $X(n, \overline{\mathcal{C}})$ parametrizing elliptic curves over $K$ with discriminant of degree $12 n$ and we show that the locus determined by $\mu=0$ is open and dense (Theorem 6.3.1).

Finally, Section 7 contains some explicit examples where we can compute the $\mu$-invariant. With Theorem 5.1.1 and Magma [Bosma et al. 1997], we can routinely and quickly calculate the $\mu$-invariant of (a quadratic base change of) an elliptic curve over a rational function field of small characteristic with mild complexity. We demonstrate this in Sections 7.3 and 7.4 among other examples.

The paper ends with the Appendix explaining the gap in the literature mentioned in Section 1.1.4 and proving a certain integrality property of the exact $p$-power factor of the $L$-function of $A$. This shows that the slope term in the formula (10) is an integer. This is not a consequence of the result $\mu_{A / K} \in \mathbb{Z}$ in Theorem 2.4.3 since (10) has not been verified unconditionally for all abelian varieties. These two types of integrality should be considered as separate results.

1.3. Notation. Let $p>0$ be a prime number. Let $K$ be a function field in one variable with field of constants $\mathbb{F}_{q}, q=p^{e}$. Write $\mathbb{F}_{q, n}, \mathbb{F}_{q, \infty}, K_{n}^{(p)}$ and $K_{\infty}^{(p)}$, for $\mathbb{F}_{q p^{n}}, \bigcup_{n} \mathbb{F}_{q, n}, K \mathbb{F}_{q, n}$ and $K \mathbb{F}_{q, \infty}$ so that $K_{n}^{(p)}$ is the $n$-th layer of $K_{\infty}^{(p)} / K$. Denote $\Gamma:=\operatorname{Gal}\left(K_{\infty}^{(p)} / K\right)$ and $\Lambda:=\mathbb{Z}_{p} \llbracket \Gamma \rrbracket$.

The cohomology groups will be usually Galois or étale cohomology, except for cohomology of finite group schemes where we use the flat cohomology, denoted $\mathrm{H}_{\mathrm{fl}}^{*}$ (see [Milne 1980, II, §1 and III, Definition 1.5] for its definition and [Milne 1986, III] for the facts needed in this paper).

Let $\mathcal{C}$ be the smooth projective curve over $\mathbb{F}_{q}$ having $K$ as its function field. Write $g_{\mathcal{C}}$ for the genus of $\mathcal{C}$. Let $A / K$ be an abelian variety. For an abelian group $H$, we denote its $p^{n}$-torsion subgroup by $H\left[p^{n}\right]$, including $n=\infty$ (so that $H\left[p^{\infty}\right]$ means the $p$-primary torsion part). However we let $A_{p^{n}}$ denote the kernel of the multiplication by $p^{n}$ on $A$ and put $A_{p^{\infty}}:=\bigcup_{n} A_{p^{n}}$. For a field extension $L / K$, denote 
the $p^{n}$-Selmer group and the $p^{n}$-Tate-Shafarevich groups $(n=\infty$ included) by

$$
\begin{aligned}
& \operatorname{Sel}_{p^{n}}(A / L):=\operatorname{Ker}\left(\mathrm{H}_{\mathrm{fl}}^{1}\left(L, A_{p^{n}}\right) \rightarrow \bigoplus_{w} \mathrm{H}^{1}\left(L_{w}, A\right)\right), \\
& \amalg_{p^{n}}(A / L):=\operatorname{Ker}\left(\mathrm{H}^{1}(L, A)\left[p^{n}\right] \rightarrow \bigoplus_{w} \mathrm{H}^{1}\left(L_{w}, A\right)\right),
\end{aligned}
$$

where $w$ runs through all places of $L$. By dropping " $\left[p^{n}\right]$ " in the latter equation, we obtain the usual definition of the (full) Tate-Shafarevich group $\amalg(A / L)$.

Let ${ }^{\vee}$ denote the Pontryagin dual.

We will denote by $\mathcal{A}$ the Néron model of $A$. The $K / \mathbb{F}_{q}$-trace of $A$ [Conrad 2006] is denoted by $\operatorname{Tr}_{K / \mathbb{F}_{q}}(A)$.

\section{The $\mu$-invariant for abelian varieties}

Let $A / K$ be an abelian variety and let $X_{A / K}$ be the Pontryagin dual of the $\operatorname{Selmer}_{\operatorname{group}} \operatorname{Sel}_{p^{\infty}}\left(A / K_{\infty}^{(p)}\right)$. Then $X_{A / K}$ is finitely generated and torsion over $\Lambda$ [Ochiai and Trihan 2009, Theorem 1.7]. From a general structure theorem for such modules, there is an exact sequence

$$
0 \rightarrow \bigoplus_{i=1}^{k} \Lambda /\left(p^{\mu_{i}}\right) \oplus \bigoplus_{j=1}^{l} \Lambda /\left(g_{j}\right) \rightarrow X_{A / K} \rightarrow F \rightarrow 0
$$

where $F$ is finite and each $g_{j}$ corresponds to a power of an irreducible distinguished polynomial in the isomorphism $\Lambda \simeq \mathbb{Z}_{p} \llbracket t \rrbracket$. The following definition is different from the convention, but it suits us well (see Theorem 2.4.3).

Definition 2.0.1. We define the $\mu$-invariant of $X_{A / K}$ as the nonnegative rational number $\mu_{A / K}$ such that

$$
q^{\mu_{A / K}}=\prod_{i=1}^{k} p^{\mu_{i}}
$$

Readers should note that on the left-hand side of (3) there is a power of $q$ but on the right-hand side there is a product of powers of $p$. We will see in Theorem 2.4.3 that $\mu_{A / K}$ is actually an integer.

The above is not specific to $X_{A / K}$. For any finitely generated torsion $\Lambda$-module $X$ appearing in this paper, we define its $\mu$-invariant by (2) and (3). It is an integer divided by $e$ in general, where $q=p^{e}$.

2.1. Base changes. The snake lemma applied to the multiplication by $p$ on (2) yields the exact sequence

$$
F[p] \rightarrow \bigoplus_{i=1}^{k} \Lambda /(p) \oplus \bigoplus_{j=1}^{l} \Lambda /\left(g_{j}, p\right) \rightarrow X_{A / K} / p X_{A / K} \rightarrow F / p F
$$

Lemma 2.1.1. $\mu_{A / K}=0$ if and only if $\operatorname{Sel}_{p}\left(A / K_{\infty}^{(p)}\right)$ is finite.

Proof. Because each $\Lambda /\left(g_{j}, p\right)$ is finite, the lemma follows via duality from the above exact sequence.

Lemma 2.1.2. If $L / K$ is a finite Galois extension with $\mu_{A / L}=0$, then $\mu_{A / K}=0$. 
Proof. The kernel of the restriction map $\operatorname{Sel}_{p}\left(A / K_{\infty}^{(p)}\right) \rightarrow \operatorname{Sel}_{p}\left(A / L_{\infty}^{(p)}\right)$ is finite since it is a subgroup of the finite group $\mathrm{H}^{1}\left(L_{\infty}^{(p)} / K_{\infty}^{(p)}, A_{p}\left(L_{\infty}^{(p)}\right)\right)$. By Lemma 2.1.1, we obtain the result.

For a Galois extension $L / K$ with $G:=\operatorname{Gal}\left(L_{\infty}^{(p)} / K_{\infty}^{(p)}\right)$, denote

$$
\mathcal{N}_{L / K}:=\operatorname{Ker}\left(\mathrm{H}_{\mathrm{fl}}^{1}\left(K_{\infty}^{(p)}, A_{p}\right) \rightarrow \bigoplus_{w} \mathrm{H}^{1}\left(K_{\infty, w}^{(p)}, A\right) / \mathrm{H}^{1}\left(G_{w}, A\left(L_{\infty, w}^{(p)}\right)\right)\right),
$$

where $w$ runs through all places of $K_{\infty}^{(p)}$.

Lemma 2.1.3. Let $L / K$ be a finite Galois p-extension. Then $\mu_{A / L}=0$, if and only $\mathcal{N}_{L / K}$ is finite.

Proof. For simplicity write $M$ for $\operatorname{Sel}_{p}(A / L)$ and let $M^{\vee}$ be the Pontryagin dual. Let $I$ be the augmentation ideal of $\mathbb{F}_{p}[G]$. If $M$ is infinite, then ${ }^{3}$ so is $M^{\vee} / I M^{\vee}$. Hence by duality $M^{G}$ is also infinite. The exact sequence

$$
0 \longrightarrow \mathcal{N}_{L / K} \cap \mathrm{H}^{1}\left(G, A_{p}(L)\right) \longrightarrow \mathcal{N}_{L / K} \longrightarrow M^{G} \longrightarrow \mathrm{H}^{2}\left(G, A_{p}(L)\right)
$$

implies $\mathcal{N}_{L / K}$ is infinite. Conversely, if $\mathcal{N}_{L / K}$ is infinite, then so is $M^{G}$.

Lemma 2.1.4. Let $L / K$ be a finite Galois p-extension with $G:=\operatorname{Gal}\left(L_{\infty}^{(p)} / K_{\infty}^{(p)}\right)$. Then $\mathcal{N}_{L / K}$ is finite if and only if $\mu_{K}=0$ and $\bigoplus_{w} \mathrm{H}^{1}\left(G_{w}, A\left(L_{\infty, w}^{(p)}\right)\right)$ is finite.

Proof. Write $W$ for $\bigoplus_{w} \mathrm{H}^{1}\left(G_{w}, A\left(L_{\infty, w}^{(p)}\right)\right), W$ is finite if and only if $W[p]$ is finite. Consider the commutative diagram

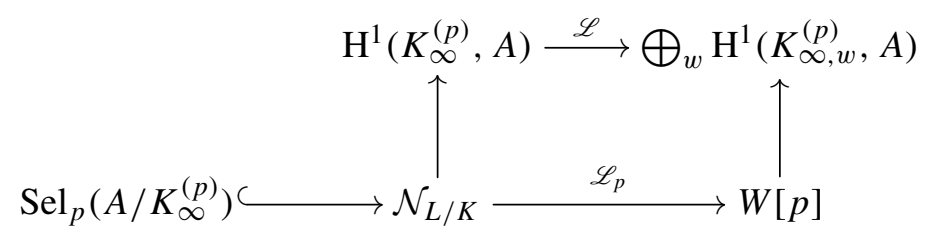

where $\mathscr{L}$ and $\mathscr{L}_{p}$ are localization maps. By using the (generalized) Cassels-Tate exact sequence [GonzálezAvilés and Tan 2007], one can deduce (see [Tan 2014, Proposition 4.2]) that $\mathscr{L}$ has cokernel of finite corank. Hence the cokernel of $\mathscr{L}_{p}$ is finite.

Theorem 2.1.5. Let $L / K$ be a finite Galois p-extension unramified outside ordinary places of $A / K$. If $\mu_{A / K}=0$, then $\mu_{A / L}=0$.

Proof. One can follow the proof of [Ochiai and Trihan 2009, Theorem 1.9], or apply Lemmas 2.1.2-2.1.4 and show that

$$
W:=\bigoplus_{w} \mathrm{H}^{1}\left(G_{w}, A\left(L_{\infty, w}^{(p)}\right)\right)
$$

has finite $p$-rank. If $L / K$ is unramified at $w$, then $\mathrm{H}^{1}\left(G_{w}, A\left(L_{\infty, w}^{(p)}\right)\right)$ is finite, and is trivial for good places (see [Milne 1986, Proposition I.3.8]). We may assume that $L / K$ is cyclic of degree $p$, so that by local class field theory, $L_{v} / K_{v}$ is an intermediate extension of some $\mathbb{Z}_{p}$-extension of $K_{v}$. By [Tan

\footnotetext{
${ }^{3}$ This should be well known for more general context. Here is a simple proof for our need. As in the commutative case, we need to show that $M / I M=0$ implies $M=0$. But this is obvious, since $G$ is a $p$-group, $I^{m}=0$ for some $m$.
} 
2010, Theorem 2(c)] and the proof of [loc. cit., Theorem 4], if $A$ has good ordinary or split multiplicative reduction at $w$, then $\mathrm{H}^{1}\left(G_{w}, A\left(L_{\infty, w}^{(p)}\right)\right)$ is finite. If $w$ is a nonsplit multiplicative place, then there is a constant field extension $K^{\prime} / K$ such that $A / K_{w}^{\prime}$ is split multiplicative. Let $L^{\prime}=K^{\prime} L$. Then the kernel of the restriction map

$$
\mathrm{H}^{1}\left(G_{w}, A\left(L_{\infty, w}^{(p)}\right)\right) \rightarrow \mathrm{H}^{1}\left(\left(L^{\prime}\right)_{\infty, w}^{(p)} /\left(K^{\prime}\right)_{\infty, w}^{(p)}, A\left(\left(L^{\prime}\right)_{\infty, w}^{(p)}\right)\right)
$$

where the target is finite, is contained in $\mathrm{H}^{1}\left(\left(K^{\prime}\right)_{\infty, w}^{(p)} / K_{\infty, w}^{(p)}, A\left(\left(K^{\prime}\right)_{\infty, w}^{(p)}\right)\right)$ which is finite, since $K^{\prime} / K$ is unramified.

2.2. The L-function. We collect here mostly well-known facts about the Hasse-Weil $L$-function of $A$ and define several invariants related to it as preparations for subsequent sections.

Let $L_{A}(s)$ be the (completed) Hasse-Weil $L$-function of $A$. See for example [Schneider 1982, $§ 1$; Kahn 2018a, V, §6] for lists of properties of $L_{A}(s)$. We can write

$$
L_{A}(s)=\frac{P_{1}(t)}{P_{0}(t) P_{2}(t)},
$$

where $P_{i}(t) \in \mathbb{Z}[t]$ is a polynomial in $t=q^{-s}$ with constant term 1 whose reciprocal roots (with multiplicities) are Weil $q$-numbers $\left\{\alpha_{i j}\right\}$ of weight $i+1$ [Kahn 2018a, V, 6.8.2].

Proposition 2.2.1. The function $P_{0}(t)^{-1}$ (with $t=q^{-s}$ ) is the zeta function of $\operatorname{Tr}_{K / \mathbb{F}_{q}}(A)$. Also, we have $P_{2}(t)=P_{0}(q t)$.

Proof. By [Schneider 1982, Satz 1], $P_{0}(t / q)$ is the characteristic polynomial of $q$-th power Frobenius on $\mathrm{V}_{l}\left(\mathcal{A}\left(\mathcal{C} \times \mathbb{F}_{q} \overline{\mathbb{F}}_{q}\right)\right)$, where $l$ is a prime different from $p$ and $\mathrm{V}_{l}$ denotes the $l$-adic Tate module tensored with $\mathbb{Q}_{l}$. We have $\mathcal{A}\left(\mathcal{C} \times_{\mathbb{F}_{q}} \overline{\mathbb{F}}_{q}\right)=A\left(K \overline{\mathbb{F}}_{q}\right)$, whose quotient by the subgroup $\operatorname{Tr}_{K / \mathbb{F}_{q}}(A)\left(\overline{\mathbb{F}}_{q}\right)$ is a finitely generated abelian group by the Lang-Néron theorem [Conrad 2006, Theorem 7.1]. Hence

$$
\mathrm{V}_{l} A\left(K \overline{\mathbb{F}}_{q}\right) \cong \mathrm{V}_{l} \operatorname{Tr}_{K / \mathbb{F}_{q}}(A)\left(\overline{\mathbb{F}}_{q}\right)
$$

This implies the statement for $P_{0}(t)$. The functional equation [Kahn 2018a, V, 6.8.1, 6.8.2] shows that $L_{A}(2-s)$ is some exponential function times $L_{A}(s)$. Hence the weight argument shows that $P_{2}(t)=P_{0}(q t)$.

Let $\lambda_{j} \in \mathbb{Q}$ be the $q$-valuation of $\alpha_{1 j}$ (where the $q$-valuation means the $p$-adic valuation such that the valuation of $q$ is 1 ).

Definition 2.2.2. We define $\theta=\theta_{A}$ to be the nonnegative rational number such that $q^{\theta} P_{1}(t / q)$ is $p$ primitive (i.e., all the coefficients are $p$-adically integral and some coefficients are $p$-adic units).

We also define $a=a_{A}:=\operatorname{deg}\left(P_{1}\right)$.

Proposition 2.2.3.

$$
\theta=\sum_{\lambda_{j}<1}\left(1-\lambda_{j}\right) \leq \frac{a}{2} .
$$


Proof. The equality can be obtained by a direct calculation or by noting that both sides are the absolute value of the height of the lowest point of the Newton polygon of the polynomial $P_{1}(t / q)$ with respect to the $q$-valuation.

The functional equation $L_{A}(s) \leftrightarrow L_{A}(2-s)$ [Kahn 2018a, V, 6.8.1, 6.8.2] implies a functional equation $P_{1}(t) \leftrightarrow P_{1}\left(q^{2} / t\right)$ since $\left\{\alpha_{i j}\right\}$ are Weil numbers. Hence the set $\left\{\alpha_{1 j}\right\}$ (with multiplicities) is equal to $\left\{q^{2} / \alpha_{1 j}\right\}$, so $\left\{\lambda_{j}\right\}=\left\{2-\lambda_{j}\right\}$. We have $\lambda_{j} \geq 0$ since $\alpha_{i j}$ are algebraic integers. Therefore

$$
\sum_{\lambda_{j}<1}\left(1-\lambda_{j}\right) \leq \sum_{\lambda_{j}<1} 1 \leq \frac{1}{2} \sum_{\text {all } \lambda_{j}} 1=\frac{\operatorname{deg}\left(P_{1}\right)}{2} .
$$

Let $N=N_{A / K}=\sum_{v} \varepsilon_{v}^{l} \cdot v$ be the global conductor of $A / K$, which is an effective divisor on $\mathcal{C}$ [Raynaud 1966, II, §3]. By the Ogg-Shafarevich formula [Raynaud 1966, II, théorème 3(ii)], we have

$$
a=\operatorname{deg}(N)+4 \operatorname{dim}(A)\left(g_{\mathcal{C}}-1\right)+4 \operatorname{dim} \operatorname{Tr}_{K / \mathbb{F}_{q}}(A) .
$$

Remark 2.2.4. The number $\theta$ is actually an integer as we will see in the Appendix. This fact will not be used below.

2.3. The Tate-Shafarevich groups. For a finitely generated torsion Iwasawa module, if its Pontryagin dual is $p$-divisible, then its $\mu$-invariant is zero. Therefore, the proposition below follows from the exact sequence

$$
0 \rightarrow A\left(K_{\infty}^{(p)}\right) \otimes \mathbb{Q}_{p} / \mathbb{Z}_{p} \rightarrow \operatorname{Sel}_{p^{\infty}}\left(A / K_{\infty}^{(p)}\right) \rightarrow \amalg_{p^{\infty}}\left(A / K_{\infty}^{(p)}\right) \rightarrow 0 .
$$

Proposition 2.3.1. The $\mu$-invariant of $\amalg_{p^{\infty}}\left(A / K_{\infty}^{(p)}\right)^{\vee}$ equals $\mu_{A / K}$.

Next, using the control theorem [Tan 2010, Theorem 4] for Selmer groups, we will give a formula for $\mu_{A / K}$ in terms of the asymptotic behavior of Tate-Shafarevich groups for finite levels $A / K_{n}^{(p)}$, as suggested by Ulmer [2019, Remark 4.3(5)], with a variant that does not assume the finiteness of Tate-Shafarevich groups.

Proposition 2.3.2. We have

for all large $m$.

$$
\mu_{A / K}=\lim _{n \rightarrow \infty} \frac{\log \left|\amalg_{p^{m}}\left(A / K_{n}^{(p)}\right)\right|}{p^{n} \log q}
$$

Proof. Let $d_{j}$ denote the degree of the polynomial $g_{j}$ in (2). Let $\sigma \in \mathrm{Gal}\left(K_{\infty}^{(p)} / K\right)$ be a topological generator. Since $\left|\Lambda /\left(p^{m}, g_{j}\right)\right|=p^{m d_{j}}$ and $\left|\Lambda /\left(p^{m}, \sigma^{p^{n}}-1\right)\right|=p^{m p^{n}}$, for every fixed $m$ one has

$$
\frac{\log \left|\operatorname{Sel}_{p^{m}}\left(A / K_{\infty}^{(p)}\right)^{\Gamma^{p^{n}}}\right|}{p^{n} \log p}=\sum_{i} \lambda_{i}+\mathrm{o}(1), \quad \text { as } n \rightarrow \infty,
$$

with $\lambda_{i}:=\min \left\{m, \mu_{i}\right\}$. By [Tan 2010, Theorem 4], we have the exact sequence

$$
0 \longrightarrow \operatorname{Ker}_{n} \longrightarrow \operatorname{Sel}_{p}\left(A / K_{n}^{(p)}\right) \longrightarrow \operatorname{Sel}_{p^{\infty}}\left(A / K_{\infty}^{(p)}\right)^{\Gamma^{p^{n}}} \longrightarrow \operatorname{Coker}_{n} \longrightarrow 0
$$


with $\left|\operatorname{Ker}_{n}\right|$ and $\left|\operatorname{Coker}_{n}\right|$ bounded as $n$ varies. For the time being, we call this the control sequence of Selmer groups. It implies

$$
\log \left|\operatorname{Sel}_{p^{m}}\left(A / K_{\infty}^{(p)}\right)^{\Gamma^{p^{n}}}\right|=\log \left|\operatorname{Sel}_{p^{m}}\left(A / K_{n}^{(p)}\right)\right|+\mathrm{O}(1), \quad \text { as } n, m \text { vary. }
$$

The rank of $A\left(K_{n}^{(p)}\right)$ is bounded. Since $\log p^{m} /\left(p^{n} \log p\right)=\mathrm{o}(1)$, the exact sequence

$$
0 \rightarrow A\left(K_{n}^{(p)}\right) \otimes \mathbb{Q}_{p} / \mathbb{Z}_{p} \rightarrow \operatorname{Sel}_{p^{\infty}}\left(A / K_{n}^{(p)}\right) \rightarrow \amalg_{p^{\infty}}\left(A / K_{n}^{(p)}\right) \rightarrow 0
$$

leads to

$$
\frac{\log \left|\amalg_{p^{m}}\left(A / K_{n}^{(p)}\right)\right|}{p^{n} \log p}=\sum_{i} \lambda_{i}+\mathrm{o}(1), \quad \text { as } n \rightarrow \infty .
$$

If $m \geq \mu_{i}$ for all $i$, then the above tends to $\sum_{i} \mu_{i}$, as $n \rightarrow \infty$.

Denote $\sigma_{n}:=\sigma^{p^{n}}, \omega_{n}=\sigma_{n}-1$ and write

$$
\omega_{n}=v_{0} \cdot v_{1} \cdots v_{n}, \quad \text { with } v_{0}=\omega_{0} \text { and } v_{i}=1+\sigma_{i-1}+\sigma_{i-1}^{2}+\cdots+\sigma_{i-1}^{p-1} \text {, for } i>0 .
$$

Then $v_{0}, v_{1}, \ldots, v_{n}$ are relatively prime irreducible elements in $\Lambda$.

Lemma 2.3.3. Let $g_{j}$ be as in (2) and set $V=\Lambda /\left(g_{j}\right)$. If $g_{j}$ is relatively prime to all $\omega_{n}$, then $V / \omega_{n} V$ is finite of order $p^{n d_{j}+\mathrm{O}(1)}$. If $g_{j}$ is a power of some $v_{i}$ and $\omega_{n}^{\prime}=\omega_{n} / v_{i}$, for $n \geq i$, then $V / \omega_{n}^{\prime} V$ is finite of order $p^{n d_{j}+\mathrm{O}(1)}$.

Proof. Since $V$ is a finite free $\mathbb{Z}_{p}$-module, if $\varphi$ is a $\mathbb{Z}_{p}$-endomorphism on $V$, then the order of $V / \varphi(V)$ equals $|\operatorname{det} \varphi|_{p}^{-1}$, where ||$_{p}$ denote the normalized $p$-adic absolute value. The proof of [Serre 1966, Theorem 8, Case iii] shows $\left|\operatorname{det} v_{l}\right|_{p}^{-1}=p^{d_{j}}$, for $l$ large enough (where Serre denoted our $v_{l}$ by $v_{l-1}^{\prime}$ ).

Proposition 2.3.4. If $\amalg_{p^{\infty}}\left(A / K_{n}^{(p)}\right)$ is finite for all $n \in \mathbb{N}$, then

$$
\mu_{A / K}=\lim _{n \rightarrow \infty} \frac{\log \left|\amalg_{p^{\infty}}\left(A / K_{n}^{(p)}\right)\right|}{p^{n} \log q} .
$$

The right-hand side is equal to the "dimension of $\amalg(A)$ " denoted as $\operatorname{dim} \amalg(A)$ in the sense of Ulmer [2019, Proposition/Definition 4.1].

Proof. Since $\amalg_{p^{\infty}}\left(A / K_{n}^{(p)}\right)$ is the $p$ cotorsion part of $\operatorname{Sel}_{p^{\infty}}\left(A / K_{n}^{(p)}\right)$, via the aforementioned control sequence, we can estimate its order by that of the $p$ cotorsion part of $\operatorname{Sel}_{p^{\infty}}\left(A / K_{\infty}^{(p)}\right)^{\Gamma^{p^{n}}}$, or by duality, the order of the $p$-primary torsion part of $X_{A / K} / \omega_{n} X_{A / K}$. In view of (2), we need to deal with the elementary $\Lambda$-module appearing in (2). If $V=\Lambda /\left(p^{\mu_{i}}\right)$, then $V / \omega_{n} V$ has order $p^{\mu_{i}} p^{n}$. In the case where $V=\Lambda /\left(g_{j}\right)$, $g_{j}=v_{i}^{l}$, the exact sequence

$$
0 \rightarrow v_{i} \Lambda /\left(v_{i}^{l}, \omega_{n}\right) \rightarrow V / \omega_{n} V \rightarrow \Lambda / v_{i} \Lambda \rightarrow 0
$$

shows $\Lambda / \nu_{i} \Lambda$ is the $\mathbb{Z}_{p}$-free quotient of $V / \omega_{n} V$, while since $v_{i}^{l-1}$ and $\omega_{n}^{\prime}$ are relatively prime,

$$
v_{i} \Lambda /\left(v_{i}^{l}, \omega_{n}\right) \simeq \Lambda /\left(v_{i}^{l-1}, \omega_{n}^{\prime}\right)
$$


is the $p$-primary torsion part. Thus, Lemma 2.3.3 says, for every $g_{j}$ in (2), with $V=\Lambda /\left(g_{j}\right)$, the order of the $p$-primary torsion part of $V / \omega_{n} V$ is bounded by $p^{n d_{j}+\mathrm{O}(1)}$. These lead to the desired

$$
\frac{\log \left|\amalg_{p^{\infty}}\left(A / K_{n}^{(p)}\right)\right|}{p^{n} \log p}=\sum_{i} \mu_{i}+\mathrm{o}(1), \quad \text { as } n \rightarrow \infty .
$$

Remark 2.3.5. (1) Without the assumption of the finiteness of Tate-Shafarevich group, the above proof shows that, if we replace $\amalg_{p^{\infty}}\left(A / K_{n}^{(p)}\right)$ by its $p$ cotorsion part, then (6) also holds.

(2) The proof of Proposition 2.3.4 is complicated due to the lack of a control theorem for $\amalg$ at this point. It is not clear how to deduce such a theorem from the (known) control theorem for Sel. The difficulties mainly come from the fact that $H^{1}\left(\Gamma^{p^{n}}, A\left(K_{\infty}^{(p)}\right) \otimes \mathbb{Q}_{p} / \mathbb{Z}_{p}\right)$ is not finite in general. In the next subsection, we will prove a control theorem for $\amalg$ without using a control theorem for Sel, from which Proposition 2.3.4 will follow.

In the next subsection, we will use $\amalg_{p^{\infty}}\left(A / K \overline{\mathbb{F}}_{q}\right)$ in addition to $\amalg_{p^{\infty}}\left(A / K_{\infty}^{(p)}\right)$. Their relation is given as follows.

Proposition 2.3.6. The $\operatorname{Gal}\left(\overline{\mathbb{F}}_{q} / \mathbb{F}_{q, \infty}\right)$-invariant part of $\amalg_{p^{\infty}}\left(A / K \overline{\mathbb{F}}_{q}\right)$ is $\amalg_{p^{\infty}}\left(A / K_{\infty}^{(p)}\right)$.

Proof. The group $\operatorname{Gal}\left(\overline{\mathbb{F}}_{q} / \mathbb{F}_{q, \infty}\right)$ is pro-prime-to- $p$. Hence the $\operatorname{Gal}\left(\overline{\mathbb{F}}_{q} / \mathbb{F}_{q, \infty}\right)$-invariant part functor on $p$-primary torsion discrete $\operatorname{Gal}\left(\overline{\mathbb{F}}_{q} / \mathbb{F}_{q, \infty}\right)$-modules is an exact functor. This implies that the $\operatorname{Gal}\left(\overline{\mathbb{F}}_{q} / \mathbb{F}_{q, \infty}\right)$ invariant part of $\mathrm{H}^{1}\left(K \overline{\mathbb{F}}_{q}, A\right)\left[p^{\infty}\right]$ is $\mathrm{H}^{1}\left(K_{\infty}^{(p)}, A\right)\left[p^{\infty}\right]$. On the other hand, we have

$$
\bigoplus_{\bar{v}} \mathrm{H}^{1}\left(\left(K \overline{\mathbb{F}}_{q}\right)_{\bar{v}}, A\right) \cong \bigoplus_{w} \mathrm{H}^{1}\left(K_{\infty, w}^{(p)} \otimes_{\mathbb{F}_{q, \infty}} \overline{\mathbb{F}}_{q}, A\right),
$$

where $\bar{v}$ and $w$ run through all places of $K \overline{\mathbb{F}}_{q}$ and $K_{\infty}^{(p)}$, respectively. (Here note that $K_{\infty, w}^{(p)} \otimes_{\mathbb{F}_{q, \infty}} \overline{\mathbb{F}}_{q}$ is not a field but a finite product of fields.) The group $\operatorname{Gal}\left(\mathbb{\mathbb { F }}_{q} / \mathbb{F}_{q, \infty}\right)$ acts on the right-hand side term by term. The $\operatorname{Gal}\left(\overline{\mathbb{F}}_{q} / \mathbb{F}_{q, \infty}\right)$-invariant part of $\mathrm{H}^{1}\left(K_{\infty, w}^{(p)} \otimes_{\mathbb{F}_{q, \infty}} \overline{\mathbb{F}}_{q}, A\right)\left[p^{\infty}\right]$ is $\mathrm{H}^{1}\left(K_{\infty, w}^{(p)}, A\right)\left[p^{\infty}\right]$ by the same reasoning as before. Now the proposition follows from the definition of Tate-Shafarevich groups.

2.4. The Tate-Shafarevich group scheme. In [Suzuki 2020], a commutative perfect group scheme

$$
\mathcal{G}^{(i)}=\mathcal{G}_{A}^{(i)}:=\boldsymbol{H}^{i}(\mathcal{C}, \mathcal{A})
$$

over $\mathbb{F}_{q}$ for each integer $i$ is defined (where perfect means that the Frobenius is an isomorphism). It is characterized by the property [Suzuki 2020, Proposition 2.7.8] that for any perfect field extension $k / \mathbb{F}_{q}$, we have a canonical isomorphism

$$
\mathcal{G}_{A}^{(i)}(k) \cong \mathrm{H}^{i}\left(\mathcal{C}_{\bar{k}}, \mathcal{A}\right)^{\mathrm{Gal}(\bar{k} / k)}
$$

functorial in the variable $k$, where $\mathcal{C}_{\bar{k}}=\mathcal{C} \times_{\mathbb{E}_{q}} \bar{k}$ denotes the base change and the cohomology is taken in the étale topology (or, equivalently, in the flat topology, as the Néron model $\mathcal{A}$ is smooth). We call $\mathcal{G}_{A}^{(1)}$ the Tate-Shafarevich scheme, because

$$
\mathcal{G}_{A}^{(1)}\left(\overline{\mathbb{F}}_{q}\right)=\mathrm{H}^{1}\left(\mathcal{C}_{\overline{\mathbb{F}}_{q}}, \mathcal{A}\right) \cong \amalg\left(A / K \overline{\mathbb{F}}_{q}\right),
$$


where the last canonical isomorphism is [Milne 1986, III, Lemma 11.5]. Taking the Gal( $\left(\overline{\mathbb{F}}_{q} / \mathbb{F}_{q, \infty}\right)$-invariant parts of the $p$-primary torsion parts of both of the sides and using Proposition 2.3.6, we have

$$
\mathcal{G}_{A}^{(1)}\left(\mathbb{F}_{q, \infty}\right)\left[p^{\infty}\right] \cong \amalg_{p^{\infty}}\left(A / K_{\infty}^{(p)}\right) .
$$

Therefore,

$$
\left(\mathcal{G}_{A}^{(1)}\left(\mathbb{F}_{q, \infty}\right)\left[p^{\infty}\right]\right)^{\vee} \cong \amalg_{p^{\infty}}\left(A / K_{\infty}^{(p)}\right)^{\vee} .
$$

The group $\mathcal{G}_{A}^{(1)}$ is the perfection (inverse limit along Frobenius morphisms) of a commutative smooth group scheme over $\mathbb{F}_{q}$ with unipotent identity component and torsion cofinite component group $\pi_{0}\left(\mathcal{G}_{A}^{(1)}\right)$ [Suzuki 2020, Theorem 3.4.1(2)]. Here cofinite means that $\pi_{0}\left(\mathcal{G}_{A}^{(1)}\right)[n]$ is finite étale for any $n \geq 1$.

\section{Definition 2.4.1.}

$$
s_{A / K}:=\operatorname{dim} \mathcal{G}_{A}^{(1)} \text {. }
$$

Proposition 2.4.2. Let $\mathcal{G}$ be a commutative smooth group scheme over $\mathbb{F}_{q}$ such that its identity component $\mathcal{G}^{0}$ is unipotent and its component group $\pi_{0}(\mathcal{G})$ is p-primary torsion and cofinite. Then the Iwasawa module $\mathcal{G}\left(\mathbb{F}_{q, \infty}\right)^{\vee}$ is finitely generated torsion over $\Lambda$, with characteristic ideal generated by $q^{\operatorname{dim}(\mathcal{G})}$ times the characteristic polynomial of the Frobenius action on the finite-dimensional $\mathbb{Q}_{p}$-vector space $\pi_{0}(\mathcal{G})\left(\mathbb{F}_{q, \infty}\right)^{\vee} \otimes_{\mathbb{Z}_{p}} \mathbb{Q}_{p}$. In particular, the $\mu$-invariant of $\mathcal{G}\left(\mathbb{F}_{q, \infty}\right)^{\vee}$ is $\operatorname{dim}(\mathcal{G})$.

Proof. Consider the connected-étale sequence $0 \rightarrow \mathcal{G}^{0} \rightarrow \mathcal{G} \rightarrow \pi_{0}(\mathcal{G}) \rightarrow 0$. This induces an exact sequence

$$
0 \rightarrow \mathcal{G}^{0}\left(\mathbb{F}_{q, \infty}\right) \rightarrow \mathcal{G}\left(\mathbb{F}_{q, \infty}\right) \rightarrow \pi_{0}(\mathcal{G})\left(\mathbb{F}_{q, \infty}\right) \rightarrow 0
$$

since $\mathrm{H}^{1}\left(\mathbb{F}_{q, \infty}, \mathcal{G}^{0}\right)$ (which is the direct limit of $\mathrm{H}^{1}\left(\mathbb{F}_{q, n}, \mathcal{G}^{0}\right)$ in $n$ ) vanishes by Lang's theorem. ${ }^{4}$ Hence we have an exact sequence

$$
0 \rightarrow \pi_{0}(\mathcal{G})\left(\mathbb{F}_{q, \infty}\right)^{\vee} \rightarrow \mathcal{G}\left(\mathbb{F}_{q, \infty}\right)^{\vee} \rightarrow \mathcal{G}^{0}\left(\mathbb{F}_{q, \infty}\right)^{\vee} \rightarrow 0
$$

of $\Lambda$-modules. Since characteristic ideals are multiplicative in short exact sequences [Coates and Sujatha 2006, Appendix A, Proposition 1], we may assume that $\mathcal{G}$ is either connected or étale. A commutative smooth connected unipotent group over $\mathbb{F}_{q}$ is a finite successive extension of copies of $\boldsymbol{G}_{a}$ [Demazure and Gabriel 1970, IV, §2, 3.9]. Therefore the connected case is further reduced to the case of $\boldsymbol{G}_{a}$ by a similar argument.

Assume that $\mathcal{G}=\boldsymbol{G}_{a}$. Then $\left(\mathbb{F}_{q, \infty}\right)^{\vee}$ is a rank one free $\mathbb{F}_{q} \llbracket \Gamma \rrbracket$-module. In particular, it is a finitely generated torsion $\Lambda$-module with characteristic ideal $(q)$. Assume that $\mathcal{G}$ is étale. Then $\mathcal{G}\left(\mathbb{F}_{q, \infty}\right)^{\vee}$ is finitely generated over $\mathbb{Z}_{p}$. Hence it is a finitely generated torsion $\Lambda$-module with characteristic ideal generated by the characteristic polynomial of the Frobenius action on the finite-dimensional $\mathbb{Q}_{p}$-vector space $\mathcal{G}\left(\mathbb{F}_{q, \infty}\right)^{\vee} \otimes_{\mathbb{Z}_{p}} \mathbb{Q}_{p}$.

Theorem 2.4.3. The $\mu$-invariant of $\left(\mathcal{G}^{(1)}\left(\mathbb{F}_{q, \infty}\right)\left[p^{\infty}\right]\right)^{\vee}$ is $s_{A / K}$. Therefore,

$$
\mu_{A / K}=s_{A / K}
$$

\footnotetext{
${ }^{4}$ Recall the statement of Lang's theorem [Demazure and Gabriel 1970, III, §5, 7.5 and 7.11]: for any commutative connected (not necessarily affine) algebraic group $G$ over a finite field $\mathbb{F}_{q}$, the cohomology $\mathrm{H}^{n}\left(\mathbb{F}_{q}, G\right)$ vanishes for all $n \geq 1$.
} 
In particular, $\mu_{A / K}$ (and hence $\operatorname{dim} \amalg(A)$ when Tate-Shafarevich groups are finite) is an integer. Proof. This follows from (8), and Propositions 2.3.1 and 2.4.2.

In the proof of Propositions 2.3.2 and 2.3.4, we have used the control theorem for Selmer groups [Tan 2010, Theorem 4]. The group schemes $\mathcal{G}^{(i)}$, their derived descent property and arithmetic duality give a control theorem for Tate-Shafarevich groups, which we are now going to see below (Proposition 2.4.4). For $n \geq 0$, consider the natural homomorphisms:

$$
\amalg\left(A / K_{n}^{(p)}\right) \stackrel{i_{n}}{\rightarrow} \mathrm{H}^{1}\left(\mathcal{C}_{\mathbb{F}_{q, n}}, \mathcal{A}\right) \stackrel{j_{n}}{\rightarrow} \mathcal{G}^{(1)}\left(\mathbb{F}_{q, n}\right) .
$$

Note that the $p$-primary part of the last term is isomorphic to $\amalg_{p^{\infty}}\left(A / K_{\infty}^{(p)}\right)^{\Gamma^{p^{n}}}$ by (7).

Proposition 2.4.4. For all $n \geq 0$, the map $i_{n}$ is injective with finite cokernel of bounded order in $n$, and the map $j_{n}$ has finite kernel and cokernel of bounded order in $n$.

Proof. The first statement follows from the exact sequence

$$
0 \rightarrow \amalg\left(A / K_{n}^{(p)}\right) \rightarrow \mathrm{H}^{1}\left(\mathcal{C}_{\mathbb{F}_{q, n}}, \mathcal{A}\right) \rightarrow \bigoplus_{v \in \mathcal{C}_{\mathbb{q}, n}} \mathrm{H}^{1}\left(\mathbb{F}_{q, n}(v), \pi_{0}\left(\mathcal{A}_{v}\right)\right)
$$

written in [Milne 1986, III, Proposition 9.2]. For the second statement, we have a canonical spectral sequence

$$
E_{2}^{i j}=\mathrm{H}^{i}\left(\mathbb{F}_{q, n}, \mathcal{G}^{(j)}\right) \Rightarrow \mathrm{H}^{i+j}\left(\mathcal{C}_{\mathbb{F}_{q, n}}, \mathcal{A}\right)
$$

by [Suzuki 2020, Proposition 2.7.8]. Hence we have an exact sequence

$$
0 \rightarrow \mathrm{H}^{1}\left(\mathbb{F}_{q, n}, \mathcal{G}^{(0)}\right) \rightarrow \mathrm{H}^{1}\left(\mathcal{C}_{\mathbb{F}_{q, n}}, \mathcal{A}\right) \rightarrow \mathcal{G}^{(1)}\left(\mathbb{F}_{q, n}\right) \rightarrow \mathrm{H}^{2}\left(\mathbb{F}_{q, n}, \mathcal{G}^{(0)}\right) \rightarrow \mathrm{H}^{2}\left(\mathcal{C}_{\mathbb{F}_{q, n}}, \mathcal{A}\right) .
$$

The natural morphism from $\mathrm{H}^{m}\left(\mathbb{F}_{q, n}, \mathcal{G}^{(0)}\right)$ to $\mathrm{H}^{m}\left(\mathbb{F}_{q, n}, \pi_{0}\left(\mathcal{G}^{(0)}\right)\right)$ is an isomorphism for any $m \geq 1$ since $\mathrm{H}^{m}\left(\mathbb{F}_{q, n},\left(\mathcal{G}^{(0)}\right)^{0}\right)=0$ by Lang's theorem. The group scheme $\pi_{0}\left(\mathcal{G}^{(0)}\right)$ over $\mathbb{F}_{q}$ is étale with group of geometric points finitely generated by [Suzuki 2020, Theorem 3.4.1(2)]. Hence $\mathrm{H}^{1}\left(\mathbb{F}_{q, n}, \pi_{0}\left(\mathcal{G}^{(0)}\right)\right)$ is finite of order bounded in $n$. Therefore the kernel of $\mathrm{H}^{1}\left(\mathcal{C}_{\mathbb{F}_{q, n}}, \mathcal{A}\right) \rightarrow \mathcal{G}^{(1)}\left(\mathbb{F}_{q, n}\right)$ is finite of order bounded in $n$.

To show that the cokernel of this map is also finite of order bounded in $n$, let $B / K$ be the abelian variety dual to $A$ with Néron model $\mathcal{B}$. Let $\mathcal{B}^{0}$ be the part of $\mathcal{B}$ with connected fibers. Let

$$
\mathcal{F}^{(i)}=\boldsymbol{H}^{i}\left(\mathcal{C}, \mathcal{B}^{0}\right)
$$

be the group scheme defined in [Suzuki 2020]. The group $\mathrm{H}^{2}\left(\mathbb{F}_{q, n}, \mathcal{G}^{(0)}\right)$ is isomorphic to

$$
\mathrm{H}^{1}\left(\mathbb{F}_{q, n}, \pi_{0}\left(\mathcal{G}^{(0)}\right) \otimes \mathbb{Q} / \mathbb{Z}\right)
$$

since Galois cohomology is torsion in positive degrees. The latter group $\mathrm{H}^{1}\left(\mathbb{F}_{q, n}, \pi_{0}\left(\mathcal{G}^{(0)}\right) \otimes \mathbb{Q} / \mathbb{Z}\right)$ is Pontryagin dual to the profinite completion of $\operatorname{Hom}\left(\pi_{0}\left(\mathcal{G}^{(0)}\right) /\right.$ tor, $\left.\mathbb{Z}\right)\left(\mathbb{F}_{q, n}\right)$ (= the group of $\mathbb{F}_{q, n}$-valued points of the dual $\mathbb{Z}$-lattice of $\pi_{0}\left(\mathcal{G}^{(0)}\right) /$ tor). The height pairing gives a nondegenerate pairing

$$
\pi_{0}\left(\mathcal{G}^{(0)}\right) / \text { tor } \times \pi_{0}\left(\mathcal{F}^{(0)}\right) / \text { tor } \rightarrow \mathbb{Z}
$$


of $\mathbb{Z}$-lattices over $\mathbb{F}_{q}$ [Suzuki 2020, Theorem 3.4.1 (6e, 7)]. Hence we have injections

$$
\pi_{0}\left(\mathcal{F}^{(0)}\right)\left(\mathbb{F}_{q, n}\right) / \text { tor } \hookrightarrow\left(\pi_{0}\left(\mathcal{F}^{(0)}\right) / \text { tor }\right)\left(\mathbb{F}_{q, n}\right) \hookrightarrow \operatorname{Hom}\left(\pi_{0}\left(\mathcal{G}^{(0)}\right) / \text { tor, } \mathbb{Z}\right)\left(\mathbb{F}_{q, n}\right)
$$

with finite cokernels of order bounded in $n$. Therefore we have a natural map

$$
\pi_{0}\left(\mathcal{F}^{(0)}\right)\left(\mathbb{F}_{q, n}\right)^{\wedge} \rightarrow \mathrm{H}^{2}\left(\mathbb{F}_{q, n}, \mathcal{G}^{(0)}\right)^{\vee}
$$

with finite cokernel of order bounded in $n$, where ${ }^{\wedge}$ denotes the profinite completion.

On the other hand, we have a canonical exact sequence

$$
0 \rightarrow \mathcal{B}^{0}\left(\mathcal{C}_{\mathbb{F}_{q, n}}\right)^{\wedge} \rightarrow \mathrm{H}^{2}\left(\mathcal{C}_{\mathbb{F}_{q, n}}, \mathcal{A}\right)^{\vee} \rightarrow \mathrm{TH}^{1}\left(\mathcal{C}_{\mathbb{F}_{q, n}}, \mathcal{B}^{0}\right) \rightarrow 0
$$

by the duality result [Suzuki 2020, Proposition 4.2.3], where T denotes the profinite Tate module. By the connected-étale sequence

$$
0 \rightarrow\left(\mathcal{F}^{(0)}\right)^{0} \rightarrow \mathcal{F}^{(0)} \rightarrow \pi_{0}\left(\mathcal{F}^{(0)}\right) \rightarrow 0
$$

and Lang's theorem (namely, $\left.\mathrm{H}^{1}\left(\mathbb{F}_{q, n},\left(\mathcal{F}^{(0)}\right)^{0}\right)=0\right)$, the map

$$
\mathcal{F}^{(0)}\left(\mathbb{F}_{q, n}\right)=\mathcal{B}^{0}\left(\mathcal{C}_{\mathbb{F}_{q, n}}\right) \rightarrow \pi_{0}\left(\mathcal{F}^{(0)}\right)\left(\mathbb{F}_{q, n}\right)
$$

is surjective.

Therefore the cokernel of the map from $\mathrm{H}^{2}\left(\mathcal{C}_{\mathbb{F}_{q, n}}, \mathcal{A}\right)^{\vee}$ to $\mathrm{H}^{2}\left(\mathbb{F}_{q, n}, \mathcal{G}^{(0)}\right)^{\vee}$ is finite of order bounded in $n$. Thus the kernel of the map from $\mathrm{H}^{2}\left(\mathbb{F}_{q, n}, \mathcal{G}^{(0)}\right)$ to $\mathrm{H}^{2}\left(\mathcal{C}_{\mathbb{F}_{q, n}}, \mathcal{A}\right)$ is finite of order bounded in $n$. This implies that the cokernel of $\mathrm{H}^{1}\left(\mathcal{C}_{\mathbb{F}_{q, n}}, \mathcal{A}\right) \rightarrow \mathcal{G}^{(1)}\left(\mathbb{F}_{q, n}\right)$ is finite of order bounded in $n$.

The above proof actually gives an explicit bound on the orders of (the $p$-primary part of) the kernel and cokernel of $i_{n}$ and $j_{n}$ for large $n$ in terms of the finite étale groups $\pi_{0}\left(\mathcal{A}_{v}\right), \pi_{0}\left(\mathcal{G}^{(0)}\right)$ tor and $\pi_{0}\left(\mathcal{F}^{(0)}\right)$ tor and the discriminant of the height pairing on $\pi_{0}\left(\mathcal{G}^{(0)}\right) /$ tor $\times \pi_{0}\left(\mathcal{F}^{(0)}\right) /$ tor.

Using this, we can give:

Another proof of Propositions 2.3.2 and 2.3.4. The $\Gamma^{p^{n}}$-invariant part of $\mathcal{G}^{(1)}\left(\mathbb{F}_{q, \infty}\right)$ is $\mathcal{G}^{(1)}\left(\mathbb{F}_{q, n}\right)$. Therefore

$$
\lim _{n \rightarrow \infty} \frac{\log \left|\mathcal{G}^{(1)}\left(\mathbb{F}_{q, n}\right)\left[p^{m}\right]\right|}{p^{n} \log q}=s_{A / K}
$$

for all large $m$ by the same argument as the first part of the proof of Proposition 2.3.2, and if $\mathcal{G}^{(1)}\left(\mathbb{F}_{q, n}\right)\left[p^{\infty}\right]$ is finite for all $n$, then

$$
\lim _{n \rightarrow \infty} \frac{\log \left|\mathcal{G}^{(1)}\left(\mathbb{F}_{q, n}\right)\left[p^{\infty}\right]\right|}{p^{n} \log q}=s_{A / K}
$$

by Iwasawa's formula [Serre 1966, Theorem 8]. By Proposition 2.4.4, we can compare the asymptotic behavior of $\left|\mathcal{G}^{(1)}\left(\mathbb{F}_{q, n}\right)\left[p^{m}\right]\right|$ and $\left|\amalg_{p^{m}}\left(A / K_{n}^{(p)}\right)\right|$. This, with Theorem 2.4.3, gives

$$
\lim _{n \rightarrow \infty} \frac{\log \left|\amalg_{p^{m}}\left(A / K_{n}^{(p)}\right)\right|}{p^{n} \log q}=\lim _{n \rightarrow \infty} \frac{\log \left|\mathcal{G}^{(1)}\left(\mathbb{F}_{q, n}\right)\left[p^{m}\right]\right|}{p^{n} \log q}=s_{A / K}=\mu_{A / K}
$$


for all large $m$, and if $\amalg_{p^{\infty}}\left(A / K_{n}^{(p)}\right)$ is finite for all $n$, then

$$
\lim _{n \rightarrow \infty} \frac{\log \left|\amalg_{p^{\infty}}\left(A / K_{n}^{(p)}\right)\right|}{p^{n} \log q}=\lim _{n \rightarrow \infty} \frac{\log \left|\mathcal{G}^{(1)}\left(\mathbb{F}_{q, n}\right)\left[p^{\infty}\right]\right|}{p^{n} \log q}=s_{A / K}=\mu_{A / K} .
$$

Remark 2.4.5. (1) Propositions 2.4.2 and 2.4.4, together with the properties of $\mathcal{G}^{(1)}$ cited after (8), give another proof that $X_{A / K}$ is finitely generated torsion over $\Lambda$, i.e., [Ochiai and Trihan 2009, Theorem 1.7].

(2) To seek for a number field analogue of the Tate-Shafarevich scheme, let $E / \mathbb{Q}$ be an elliptic curve and $p$ a prime number. Assume that $\operatorname{Sel}_{p^{\infty}}\left(E / \mathbb{Q}^{\text {cyc }}\right)^{\vee}$ is torsion over $\mathbb{Z}_{p} \llbracket \operatorname{Gal}\left(\mathbb{Q}^{\text {cyc }} / \mathbb{Q}\right) \rrbracket$. Does there exist the perfection of some smooth group scheme over $\mathbb{F}_{p}$ whose group of $\mathbb{F}_{p, \infty}$-valued points is isomorphic to $\amalg_{p^{\infty}}\left(E / \mathbb{Q}^{\text {cyc }}\right)$ as $\mathbb{Z}_{p} \llbracket \operatorname{Gal}\left(\mathbb{Q}^{\text {cyc }} / \mathbb{Q}\right) \rrbracket \cong \mathbb{Z}_{p} \llbracket \operatorname{Gal}\left(\mathbb{F}_{p, \infty} / \mathbb{F}_{p}\right) \rrbracket$-modules?

2.5. The $\mu$-invariant formula. With the formula (6) or (9) and Proposition 2.2.3, we can now reinterpret Proposition 4.2 of [Ulmer 2019] as follows:

Corollary 2.5.1. Assume that $\amalg\left(A / K_{n}^{(p)}\right)$ is finite for all $n$. We have

$$
\mu_{A / K}=\operatorname{deg}(\mathcal{L})+\operatorname{dim}(A)\left(g_{\mathcal{C}}-1\right)+\operatorname{dim}\left(\operatorname{Tr}_{K / \mathbb{F}_{q}}(A)\right)-\theta,
$$

where $\mathcal{L}$ is the invertible sheaf on $\mathcal{C}$ defined by

$$
\mathcal{L}:=o^{*} \Omega_{\mathcal{A} / \mathcal{C}}^{\operatorname{dim}(A)}
$$

(here o $: \mathcal{C} \rightarrow \mathcal{A}$ denotes the zero section of $\mathcal{A} \rightarrow \mathcal{C}$ ).

We will prove below that the formula (10) also holds for the following two cases without any assumption on $\amalg$ : $A$ is the Jacobian of a projective smooth curve over $K$; and $A$ is a semistable abelian variety over $K$.

Remark 2.5.2. As in Proposition 2.3.4, Ulmer [2019, 4.1] called the right-hand side of (6) the dimension of $\amalg(A)$. He justified this terminology [Ulmer 2019, 4.3(2)] in the special case that $A$ is a Jacobian. For a general abelian variety $A$, our definition $s_{A / K}=\operatorname{dim} \mathcal{G}_{A}^{(1)}$ and the formula (9) together justify the terminology.

The term $\operatorname{deg}(\mathcal{L})$ is nonnegative [Yuan 2018, Theorem 2.6]. Also, recall that the term $\theta$ in the formula of the above corollary is nonnegative so that we get the following upper bound on the $\mu$-invariant (see [Ulmer 2019, Proposition 4.4] for another upper bound for $\mu$ ):

$$
\mu_{A / K} \leq \operatorname{deg}(\mathcal{L})+\operatorname{dim}(A)\left(g_{\mathcal{C}}-1\right)+\operatorname{dim}\left(\operatorname{Tr}_{K / \mathbb{F}_{q}}(A)\right) .
$$

About the following definition, see also Theorem 5.2.1 below.

Definition 2.5.3. Define the Szpiro difference as

$$
d=d_{A}:=\frac{\operatorname{deg}(N)}{2}+\operatorname{dim}(A)\left(g_{\mathcal{C}}-1\right)+\operatorname{dim} \operatorname{Tr}_{K / \mathbb{F}_{q}}(A)-\operatorname{deg}(\mathcal{L}) .
$$


Also define $b=b_{A}:=a / 2-\theta$.

Note that $b$ is nonnegative by Proposition 2.2.3 and defined purely from the $L$-function of $A$. By (4), we can rewrite the formula (10) in terms of $b$ and $d$ :

Corollary 2.5.4. The formula (10) is equivalent to the formula $\mu_{A / K}=b-d$.

Remark 2.5.5. The formula (10) can be understood as a certain kind of Euler characteristic formula for $L_{A}(s)$ at $s=1$. To see this, let $\operatorname{Lie}(\mathcal{A})$ be the vector bundle on $\mathcal{C}$ dual to $o^{*} \Omega_{\mathcal{A} / \mathcal{C}}$. Then by the Riemann-Roch theorem, we have

$$
\chi(\mathcal{C}, \operatorname{Lie}(\mathcal{A})):=\sum_{n}(-1)^{n} \operatorname{dim}_{\mathbb{F}_{q}} \mathrm{H}^{n}(\mathcal{C}, \operatorname{Lie}(\mathcal{A}))=\operatorname{dim}(A)\left(1-g_{\mathcal{C}}\right)-\operatorname{deg}(\mathcal{L}) .
$$

Also let

$$
\chi^{0}(\mathcal{C}, \mathcal{A}):=\sum_{n}(-1)^{n} \operatorname{dim} \mathcal{G}_{A}^{(n)} .
$$

By [Suzuki 2020, Propositions 3.2.2, 3.2.3, Theorem 3.4.1(2)], we have $\operatorname{dim} \mathcal{G}_{A}^{(0)}=\operatorname{dim} \operatorname{Tr}_{K / \mathbb{F}_{q}}(A)$ and $\operatorname{dim} \mathcal{G}_{A}^{(n)}=0$ for $n \neq 0,1$. Therefore the formula (10) can be written as

$$
\theta=\chi^{0}(\mathcal{C}, \mathcal{A})-\chi(\mathcal{C}, \operatorname{Lie}(\mathcal{A}))
$$

Compare this with the Weil-étale BSD formula [Geisser and Suzuki 2020, Proposition 8.4]. This presentation might be useful when one wants to generalize (10), for example to motives over $K$ other than abelian varieties.

\section{The $\mu$-invariant for Jacobians}

In this section, we show that the formula (10) holds for Jacobians without any hypothesis (Corollary 3.4.1) and deduce some necessary and sufficient conditions about the (non)vanishing of the $\mu$-invariant (Propositions 3.4.2 and 3.4.4). A good reference on fibered surfaces is [Liu 2002]. Let $\mathcal{S}$ be a projective smooth surface over $\mathbb{F}_{q}$ and $\pi: \mathcal{S} \rightarrow \mathcal{C}$ a flat morphism over $\mathbb{F}_{q}$. Assume that $\pi_{*} \mathcal{O}_{\mathcal{S}}=\mathcal{O}_{\mathcal{C}}$ and that the generic fiber $\pi_{K}: \mathcal{S}_{K} \rightarrow \operatorname{Spec} K$ of $\pi$ is smooth. Let $A=\operatorname{Pic}_{\mathcal{S}_{K} / K}^{0}$ be the Jacobian variety of $\mathcal{S}_{K}$ over $K$. Any elliptic curve is an example of such $A$ by the theory of regular models. We do not assume that $\pi$ admits a section.

3.1. The Brauer group. Recall from [Artin 1974b, §3; Milne 1976, §5] that there is a perfect group scheme $\underline{\mathrm{H}}^{i}\left(p^{\infty}\right)$ over $\mathbb{F}_{q}$ for each integer $i$ such that for any perfect field extension $k / \mathbb{F}_{q}$, we have

$$
\underline{\mathrm{H}}^{i}\left(p^{\infty}\right)(k)=\mathrm{H}_{\mathrm{fl}}^{i}\left(\mathcal{S}_{\bar{k}}, \mu_{p^{\infty}}\right)^{\mathrm{Gal}(\bar{k} / k)}
$$

as a functor in the variable $k$, where $\mathcal{S}_{\bar{k}}=\mathcal{S} \times_{\mathbb{F}_{q}} \bar{k}$. Its identity component and component group are denoted by $\underline{\mathrm{U}}^{i}\left(p^{\infty}\right)$ and $\underline{\mathrm{D}}^{i}\left(p^{\infty}\right)$, respectively. The group $\underline{\mathrm{U}}^{i}\left(p^{\infty}\right)$ is the perfection of a commutative unipotent algebraic group over $\mathbb{F}_{q}$. The group $\underline{\mathrm{D}}^{i}\left(p^{\infty}\right)$ is a $p$-primary torsion cofinite étale group scheme over $\mathbb{F}_{q}$.

Let $\underline{\mathrm{NS}}=\pi_{0}\left(\operatorname{Pic}_{\mathcal{S} / \mathbb{F}_{q}}\right)$. It is an étale group scheme over $\mathbb{F}_{q}$ such that its group of $\overline{\mathbb{F}}_{q}$-points is the Néron-Severi group $\mathrm{NS}\left(\mathcal{S}_{\overline{\mathbb{F}}_{q}}\right)$ of $\mathcal{S}_{\overline{\mathbb{F}}_{q}}$, which is a finitely generated abelian group. The Kummer sequence 
defines a canonical injection $\underline{\mathrm{NS}} \otimes \mathbb{Q}_{p} / \mathbb{Z}_{p} \hookrightarrow \underline{\mathrm{H}}^{2}\left(p^{\infty}\right)$ of perfect group schemes. Define $\underline{\mathrm{Br}}_{p^{\infty}}$ to be its cokernel (a priori as a sheaf on the fppf site or the big étale site of $\mathbb{F}_{q}$ ).

Proposition 3.1.1. The sheaf $\underline{\mathrm{Br}}_{p^{\infty}}$ is represented by a perfect group scheme over $\mathbb{F}_{q}$. Its identity component is a quotient of $\underline{\mathrm{U}}^{2}\left(p^{\infty}\right)$ by a finite étale group.

Proof. We have an exact sequence

$$
0 \rightarrow \frac{\underline{\mathrm{U}}^{2}\left(p^{\infty}\right)}{\left(\underline{\mathrm{NS}} \otimes \mathbb{Q}_{p} / \mathbb{Z}_{p}\right) \cap \underline{\mathrm{U}}^{2}\left(p^{\infty}\right)} \rightarrow \underline{\mathrm{Br}}_{p^{\infty}} \rightarrow \frac{\underline{\mathrm{D}}^{2}\left(p^{\infty}\right)}{\underline{\mathrm{NS}} \otimes \mathbb{Q}_{p} / \mathbb{Z}_{p}} \rightarrow 0 .
$$

The intersection $\left(\underline{\mathrm{NS}} \otimes \mathbb{Q}_{p} / \mathbb{Z}_{p}\right) \cap \underline{\mathrm{U}}^{2}\left(p^{\infty}\right)$ is a finite étale group since the unipotent group $\underline{\mathrm{U}}^{2}\left(p^{\infty}\right)$ over $\mathbb{F}_{q}$ is killed by a power of $p$. Hence the first term in the above exact sequence is the perfection of a connected unipotent algebraic group. The third term is an étale group scheme. Therefore $\underline{\operatorname{Br}} p_{\infty}$ is represented by a perfect group scheme, and the above exact sequence is its connected-étale sequence.

For a prime $l \neq p$, define $\underline{\mathrm{Br}}_{l^{\infty}}$ to be the étale group scheme over $\mathbb{F}_{q}$ whose group of $\overline{\mathbb{F}}_{q}$-points is $\operatorname{Br}\left(\mathcal{S}_{\overline{\mathbb{F}}_{q}}\right)\left[l^{\infty}\right]$, where $\mathrm{Br}$ denotes the usual Brauer group for schemes. Define $\underline{\operatorname{Br}}$ to be the direct sum of $\underline{\mathrm{Br}}_{l}$ over all primes $l$, which is again a perfect group scheme. For any perfect field extension $k / \mathbb{F}_{q}$, we have a canonical isomorphism

$$
\underline{\operatorname{Br}}(k) \cong \operatorname{Br}\left(\mathcal{S}_{\bar{k}}\right) \operatorname{Gal}(\bar{k} / k)
$$

functorial in $k$.

Remark 3.1.2. There is a natural map $\operatorname{Br}(\mathcal{S}) \rightarrow \underline{\operatorname{Br}}\left(\mathbb{F}_{q}\right)$. It can be shown that the kernel and cokernel of this map are finite, and the kernel and cokernel of the map $\operatorname{Br}\left(\mathcal{S}_{\mathbb{F}_{q, n}}\right) \rightarrow \underline{\operatorname{Br}}\left(\mathbb{F}_{q, n}\right)$ are finite of order bounded in $n$, by an argument similar to the proof of Proposition 2.4.4, using [Milne 1976] instead of [Suzuki 2020]. An explicit bound for large $n$ involves the order of the torsion part of $\underline{N S}$ and the discriminant of the intersection pairing on NS/tor. We do not use this fact, so we do not prove it. See [Artin 1974b, Section 6] for a discussion about this map in the special case of an elliptic supersingular K3 surface over a large enough finite field. ${ }^{5}$

Definition 3.1.3. Define $s_{\mathcal{S}}$ to be the common integer

$$
\operatorname{dim} \underline{H}^{i}\left(p^{\infty}\right)=\operatorname{dim} \underline{U}^{i}\left(p^{\infty}\right)=\operatorname{dim} \underline{\operatorname{Br}} p^{\infty}=\operatorname{dim} \underline{\operatorname{Br}} .
$$

Remark 3.1.4. Note that the integer $s_{\mathcal{S}}$ is also equal to the length of

$$
\mathrm{H}^{2}\left(\mathcal{S}, W \mathcal{O}_{\mathcal{S}}\right)\left[p^{\infty}\right] \otimes_{W \llbracket V \rrbracket} W((V))
$$

as a $W((V))$-module, where $W=W\left(\mathbb{F}_{q}\right)$ and $V$ denotes the Verschiebung; see [Milne 1975, (1.3)] (see also [Milne and Ramachandran 2015, Lemma 2.1, Proposition 4.4]). If the formal Brauer group of $\mathcal{S}$ is pro-representable by a formal Lie group (such as when $\operatorname{Pic}_{\mathcal{S} / \mathbb{F}_{q}}$ is smooth [Artin and Mazur 1977, (4.1)]),

\footnotetext{
${ }^{5}$ Note however that [Artin 1974b, (6.1)] claims that the $\operatorname{map} \operatorname{Br}(\mathcal{S}) \rightarrow \underline{\operatorname{Br}}\left(\mathbb{F}_{q}\right)$ is bijective for such $\mathcal{S}$. This is not correct: the map is injective, but the cokernel is isomorphic to the discriminant group $\mathrm{NS}(\mathcal{S})^{*} / \mathrm{NS}(\mathcal{S}) \neq 0$ of the intersection pairing on $\mathcal{S}$, as everywhere else in [Artin 1974b, Section 6] correctly suggests.
} 
then its Dieudonné module is given by $\mathrm{H}^{2}\left(\mathcal{S}, W \mathcal{O}_{\mathcal{S}}\right)$ [Artin and Mazur 1977, II, (4.3)]. Therefore in this case, $s_{\mathcal{S}}$ is also equal to the dimension of the unipotent part of the formal Brauer group.

Proposition 3.1.5. There exists a canonical morphism $\mathcal{G}_{A}^{(1)} \rightarrow \underline{\operatorname{Br}}$ of perfect group schemes over $\mathbb{F}_{q}$ with finite étale kernel and cokernel. In particular,

$$
s_{A / K}=s_{\mathcal{S}}
$$

(which is equal to $\mu_{A / K}$ by Theorem 2.4.3).

Proof. Let $j: \operatorname{Spec} K \hookrightarrow \mathcal{C}$ be the inclusion. Let $\operatorname{Pic}_{\mathcal{S} / \mathcal{C}}$ and $\operatorname{Pic}_{\mathcal{S}_{K} / K}$ be the Picard functors sheafified in the étale topology [Kleiman 2005, Definition 2.2; Bosch et al. 1990, 8.1]. For any perfect field extension $k / \mathbb{F}_{q}$, we have canonical homomorphisms

$$
\operatorname{Br}\left(\mathcal{S}_{k}\right) \rightarrow \mathrm{H}^{1}\left(\mathcal{C}_{k}, \operatorname{Pic}_{\mathcal{S} / \mathcal{C}}\right) \rightarrow \mathrm{H}^{1}\left(\mathcal{C}_{k}, j_{*} \operatorname{Pic}_{\mathcal{S}_{K} / K}\right)
$$

functorial in $k$, which are isomorphisms if $k$ is algebraically closed, by [Grothendieck 1968, Proposition (4.3), Equation (4.14 bis)].

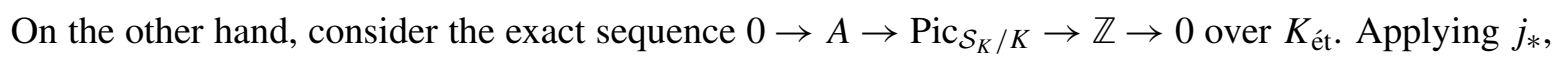
we have an exact sequence $0 \rightarrow \mathcal{A} \rightarrow j_{*} \operatorname{Pic}_{\mathcal{S}_{K} / K} \rightarrow \mathbb{Z}$ over $\mathcal{C}_{\text {ét }}$. Let $Q$ be the image of $j_{*} \operatorname{Pic}_{\mathcal{S}_{K} / K} \rightarrow \mathbb{Z}$, which is a $\mathbb{Z}$-constructible étale subsheaf of $\mathbb{Z}$ on $\mathcal{C}$. The quotient $\mathbb{Z} / Q$ is a skyscraper sheaf with finite stalks. Consider the exact sequence $0 \rightarrow \mathcal{A} \rightarrow j_{*} \operatorname{Pic}_{\mathcal{S}_{K} / K} \rightarrow Q \rightarrow 0$. For any perfect field extension $k / \mathbb{F}_{q}$, this induces an exact sequence

$$
Q\left(\mathcal{C}_{k}\right) \rightarrow \mathrm{H}^{1}\left(\mathcal{C}_{k}, \mathcal{A}\right) \rightarrow \mathrm{H}^{1}\left(\mathcal{C}_{k}, j_{*} \operatorname{Pic}_{\mathcal{S}_{K} / K}\right) \rightarrow \mathrm{H}^{1}\left(\mathcal{C}_{k}, Q\right)
$$

functorial in $k$. The image of $Q\left(\mathcal{C}_{k}\right) \rightarrow \mathrm{H}^{1}\left(\mathcal{C}_{k}, \mathcal{A}\right)$ is torsion since $\mathrm{H}^{1}\left(\mathcal{C}_{k}, \mathcal{A}\right)$ is torsion by the proof of [Milne 1986, III, Lemma 11.5]. If $k$ is algebraically closed, then $Q\left(\mathcal{C}_{k}\right)$ is finitely generated and $\mathrm{H}^{1}\left(\mathcal{C}_{k}, Q\right)$ is finite by the properties of $Q$ seen above. Therefore as functors in $k$, the kernel and cokernel of

$$
\mathrm{H}^{1}\left(\mathcal{C}_{\bar{k}}, \mathcal{A}\right)^{\mathrm{Gal}(\bar{k} / k)} \rightarrow \mathrm{H}^{1}\left(\mathcal{C}_{\bar{k}}, j_{*} \operatorname{Pic}_{\mathcal{S}_{K} / K}\right)^{\mathrm{Gal}(\bar{k} / k)}
$$

are represented by finite étale group schemes over $\mathbb{F}_{q}$.

Now we have a homomorphism and an isomorphism

$$
\mathrm{H}^{1}\left(\mathcal{C}_{\bar{k}}, \mathcal{A}\right)^{\operatorname{Gal}(\bar{k} / k)} \rightarrow \mathrm{H}^{1}\left(\mathcal{C}_{\bar{k}}, j_{*} \operatorname{Pic}_{\mathcal{S}_{K} / K}\right)^{\operatorname{Gal}(\bar{k} / k)} \sim \operatorname{Br}\left(\mathcal{S}_{\bar{k}}\right)^{\operatorname{Gal}(\bar{k} / k)}
$$

functorial in $k$. This gives the required morphism $\mathcal{G}_{A}^{(1)} \rightarrow \underline{\mathrm{Br}}$.

\subsection{The trace and the Picard variety.}

Proposition 3.2.1. Let $\operatorname{Pic}_{\mathcal{S} / \mathbb{F}_{q} \text {, red }}^{0}$ be the reduced part of $\mathrm{Pic}_{\mathcal{S} / \mathbb{F}_{q}}^{0}$. Then there exist canonical morphisms $\operatorname{Pic}_{\mathcal{C} / \mathbb{F}_{q}}^{0} \rightarrow \operatorname{Pic}_{\mathcal{S} / \mathbb{F}_{q}, \text { red }}^{0} \rightarrow \operatorname{Tr}_{K / \mathbb{F}_{q}}(A)$ over $\mathbb{F}_{q}$, which induce an exact sequence

$$
0 \rightarrow \operatorname{Pic}_{\mathcal{C} / \mathbb{F}_{q}}^{0} \rightarrow \operatorname{Pic}_{\mathcal{S} / \mathbb{F}_{q}, \text { red }}^{0} \rightarrow \operatorname{Tr}_{K / \mathbb{F}_{q}}(A) \rightarrow 0
$$


in the category of abelian varieties up to isogeny over $\mathbb{F}_{q}$. In particular, we have

$$
\operatorname{dim} \operatorname{Pic}_{\mathcal{S} / \mathbb{F}_{q}, \text { red }}^{0}=\operatorname{dim} \operatorname{Tr}_{K / \mathbb{F}_{q}}(A)+g_{\mathcal{C}}
$$

Proof. The morphism $\operatorname{Pic}_{\mathcal{C} / \mathbb{F}_{q}}^{0} \rightarrow \operatorname{Pic}_{\mathcal{S} / \mathbb{F}_{q} \text {,red }}^{0}$ is the obvious one. To define the second morphism, consider the inclusion $\mathcal{S}_{K}=\mathcal{S} \times_{\mathcal{C}} K \hookrightarrow \mathcal{S} \times_{\mathbb{F}_{q}} K$ over $K$. This induces a morphism

$$
\left(\operatorname{Pic}_{\mathcal{S} / \mathbb{F}_{q}}\right) \times_{\mathbb{F}_{q}} K=\operatorname{Pic}_{\left(\mathcal{S} \times \mathbb{F}_{q} K\right) / K} \rightarrow \operatorname{Pic}_{\mathcal{S}_{K} / K}
$$

and hence a morphism

$$
\left(\operatorname{Pic}_{\mathcal{S} / \mathbb{F}_{q}, \text { red }}^{0}\right) \times_{\mathbb{F}_{q}} K \rightarrow \operatorname{Pic}_{\mathcal{S}_{K} / K}^{0}=A
$$

over $K$. The universal property of the trace then induces a morphism $\operatorname{Pic}_{\mathcal{S} / \mathbb{F}_{q}, \text { red }}^{0} \rightarrow \operatorname{Tr}_{K / \mathbb{F}_{q}}(A)$ over $\mathbb{F}_{q}$.

To prove the second claim, it is enough to show that these morphisms induce an exact sequence

$$
0 \rightarrow \operatorname{Pic}_{\mathcal{C} / \mathbb{F}_{q}}^{0}\left(\overline{\mathbb{F}}_{q}\right) \rightarrow \operatorname{Pic}_{\mathcal{S} / \mathbb{F}_{q}}^{0}\left(\overline{\mathbb{F}}_{q}\right) \rightarrow \operatorname{Tr}_{K / \mathbb{F}_{q}}(A)\left(\overline{\mathbb{F}}_{q}\right) \rightarrow 0
$$

up to finite groups. By [Grothendieck 1968, Equation (4.14)], there exists a canonical exact sequence

$$
0 \rightarrow E \rightarrow \operatorname{Pic}_{\mathcal{S} / \mathcal{C}}\left(\mathcal{C}_{\overline{\mathbb{F}}_{q}}\right) \rightarrow \operatorname{Pic}_{\mathcal{S}_{K / K}}\left(K \overline{\mathbb{F}}_{q}\right) \rightarrow 0
$$

for some finitely generated group $E$. Since $\operatorname{Br}\left(\mathcal{C}_{\overline{\mathbb{F}}_{q}}\right)=0$, we have a canonical exact sequence

$$
0 \rightarrow \operatorname{Pic}\left(\mathcal{C}_{\overline{\mathbb{F}}_{q}}\right) \rightarrow \operatorname{Pic}\left(\mathcal{S}_{\overline{\mathbb{F}}_{q}}\right) \rightarrow \operatorname{Pic} \mathcal{S}_{\mathcal{S}}\left(\mathcal{C}_{\overline{\mathbb{F}}_{q}}\right) \rightarrow 0
$$

by [Grothendieck 1968, Equation (4.5)]. Hence we have an exact sequence

$$
0 \rightarrow \operatorname{Pic}\left(\mathcal{C}_{\overline{\mathbb{F}}_{q}}\right) \oplus E \rightarrow \operatorname{Pic}\left(\mathcal{S}_{\overline{\mathbb{F}}_{q}}\right) \rightarrow \operatorname{Pic}_{\mathcal{S}_{K / K}}\left(K \overline{\mathbb{F}}_{q}\right) \rightarrow 0 .
$$

The group $\operatorname{Pic}_{\mathcal{S} / \mathbb{F}_{q}}^{0}\left(\overline{\mathbb{F}}_{q}\right)$ is the divisible subgroup of $\operatorname{Pic}\left(\mathcal{S}_{\overline{\mathbb{F}}_{q}}\right)$ and the quotient of $\operatorname{Pic}\left(\mathcal{S}_{\overline{\mathbb{F}}_{q}}\right)$ by $\operatorname{Pic}_{\mathcal{S} / \mathbb{F}_{q}}^{0}\left(\overline{\mathbb{F}}_{q}\right)$ is $\operatorname{NS}\left(\mathcal{S}_{\overline{\mathbb{F}}_{q}}\right)$, which is finitely generated. On the other hand, the group $\operatorname{Tr}_{K / \mathbb{F}_{q}}(A)\left(\overline{\mathbb{F}}_{q}\right)$ is the divisible subgroup of $A\left(K \overline{\mathbb{F}}_{q}\right)$ and the quotient of $A\left(K \overline{\mathbb{F}}_{q}\right)$ by $\operatorname{Tr}_{K / \mathbb{F}_{q}}(A)\left(\overline{\mathbb{F}}_{q}\right)$ is finitely generated by the LangNéron theorem [Conrad 2006, Theorem 7.1]. Since $\operatorname{Pic}_{\mathcal{S}_{K} / K}\left(K \overline{\mathbb{F}}_{q}\right) / A\left(K \overline{\mathbb{F}}_{q}\right)$ injects into $\mathbb{Z}$, this implies that the group $\operatorname{Tr}_{K / \mathbb{F}_{q}}(A)\left(\overline{\mathbb{F}}_{q}\right)$ is the divisible subgroup of $\operatorname{Pic}_{\mathcal{S}_{K} / K}\left(K \overline{\mathbb{F}}_{q}\right)$ and the quotient of $\operatorname{Pic}_{\mathcal{S}_{K} / K}\left(K \overline{\mathbb{F}}_{q}\right)$ by $\operatorname{Tr}_{K / \mathbb{F}_{q}}(A)\left(\overline{\mathbb{F}}_{q}\right)$ is finitely generated. Therefore the above sequence induces an exact sequence

$$
0 \rightarrow \operatorname{Pic}_{\mathcal{C} / \mathbb{F}_{q}}^{0}\left(\overline{\mathbb{F}}_{q}\right) \rightarrow \operatorname{Ker}\left(\operatorname{Pic}_{\mathcal{C} / \mathbb{F}_{q}}^{0}\left(\overline{\mathbb{F}}_{q}\right) \rightarrow \operatorname{Tr}_{K / \mathbb{F}_{q}}(A)\left(\overline{\mathbb{F}}_{q}\right)\right) \rightarrow \mathbb{Z} \oplus E,
$$

and the morphism from the middle kernel term to $\mathbb{Z} \oplus E$ has finite image. This proves the second claim.

3.3. The L-function and the zeta function. Write the zeta function of $\mathcal{S}$ as

$$
Z(\mathcal{S}, s)=\frac{P_{\mathcal{S}, 1}(t) P_{\mathcal{S}, 3}(t)}{P_{\mathcal{S}, 0}(t) P_{\mathcal{S}, 2}(t) P_{\mathcal{S}, 4}(t)},
$$

where $P_{\mathcal{S}, i}(t) \in \mathbb{Z}[t]$ is a polynomial in $t=q^{-s}$ with constant term 1 whose reciprocal roots are Weil $q$-numbers $\left\{\alpha_{\mathcal{S}, i j}\right\}$ of weight $i$. Define $\theta_{\mathcal{S}}$ to be the nonnegative number such that $q^{\theta_{\mathcal{S}}} P_{\mathcal{S}, 2}(t / q)$ is 
$p$-primitive. Let $\lambda_{\mathcal{S}, j}$ be the $q$-valuation of $\alpha_{\mathcal{S}, 2 j}$. Then again we have

$$
\theta_{\mathcal{S}}=\sum_{\lambda_{\mathcal{S}, j}<1}\left(1-\lambda_{\mathcal{S}, j}\right)
$$

Proposition 3.3.1. The zeros and poles of the rational function $P_{2, \mathcal{S}}(t / q) / P_{1}(t / q)$ are roots of unity. In particular, we have $\theta_{\mathcal{S}}=\theta_{A}$.

Proof. Let $l \neq p$ be a prime number. We have an exact sequence

$$
0 \rightarrow \mathrm{NS}\left(\mathcal{S}_{\overline{\mathbb{F}}_{q}}\right) \otimes \mathbb{Q}_{l} \rightarrow \mathrm{H}^{2}\left(\mathcal{S}_{\overline{\mathbb{F}}_{q}}, \mathbb{Q}_{l}(1)\right) \rightarrow \mathrm{V}_{l}\left(\operatorname{Br}\left(\mathcal{S}_{\overline{\mathbb{F}}_{q}}\right)\right) \rightarrow 0
$$

of $l$-adic representations over $\mathbb{F}_{q}$, where $\mathrm{V}_{l}$ denotes the $l$-adic Tate module tensored with $\mathbb{Q}_{l}$ and the cohomology in the middle term is the continuous cohomology. The characteristic polynomial of geometric Frobenius on the middle term $\mathrm{H}^{2}\left(\mathcal{S}_{\overline{\mathbb{F}}_{q}}, \mathbb{Q}_{l}(1)\right)$ is $P_{\mathcal{S}, 2}(t / q)$. Since the Galois action on the left term $\operatorname{NS}\left(\mathcal{S}_{\overline{\mathbb{F}}_{q}}\right) \otimes \mathbb{Q}_{l}$ factors through a finite quotient, its Frobenius eigenvalues are roots of unity.

On the other hand, let $\mathcal{A}^{0}$ be the part of $\mathcal{A}$ with connected fibers. For any $n \geq 0$, the sequence $0 \rightarrow \mathcal{A}^{0}\left[l^{n}\right] \rightarrow \mathcal{A}^{0} \stackrel{l^{n}}{\rightarrow} \mathcal{A}^{0} \rightarrow 0$ is exact by [Bosch et al. 1990, 7.3/1,2]. Hence it induces an exact sequence

$$
0 \rightarrow \mathcal{A}^{0}\left(\mathcal{C}_{\overline{\mathbb{F}}_{q}}\right) \otimes \mathbb{Z} / l^{n} \mathbb{Z} \rightarrow \mathrm{H}^{1}\left(\mathcal{C}_{\overline{\mathbb{F}}_{q}}, \mathcal{A}^{0}\left[l^{n}\right]\right) \rightarrow \mathrm{H}^{1}\left(\mathcal{C}_{\overline{\mathbb{F}}_{q}}, \mathcal{A}^{0}\right)\left[l^{n}\right] \rightarrow 0
$$

of $\mathbb{Z} / l^{n} \mathbb{Z}$-representations over $\mathbb{F}_{q}$. Taking the inverse limit in $n$ and the tensor product with $\mathbb{Q}_{l}$, we have an exact sequence

$$
0 \rightarrow \frac{A\left(K \overline{\mathbb{F}}_{q}\right)}{\operatorname{Tr}_{K / \mathbb{F}_{q}}(A)\left(\overline{\mathbb{F}}_{q}\right)} \otimes \mathbb{Q}_{l} \rightarrow \mathrm{H}^{1}\left(\mathcal{C}_{\overline{\mathbb{F}}_{q}}, \mathrm{~V}_{l}(\mathcal{A})\right) \rightarrow \mathrm{V}_{l}\left(\mathrm{H}^{1}\left(\mathcal{C}_{\overline{\mathbb{F}}_{q}}, \mathcal{A}\right)\right) \rightarrow 0
$$

of $l$-adic representations over $\mathbb{F}_{q}$. By [Schneider 1982, Satz 1], the characteristic polynomial of geometric Frobenius on the middle term $\mathrm{H}^{1}\left(\mathcal{C}_{\overline{\mathbb{F}}_{q}}, \mathrm{~V}_{l}(\mathcal{A})\right)$ is $P_{1}(t / q)$. Since the Galois action on the left term $A\left(K \overline{\mathbb{F}}_{q}\right) / \operatorname{Tr}_{K / \mathbb{F}_{q}}(A)\left(\overline{\mathbb{F}}_{q}\right) \otimes \mathbb{Q}_{l}$ factors through a finite quotient, its Frobenius eigenvalues are roots of unity.

Now the morphism in Proposition 3.1.5 induces an isomorphism

$$
\mathrm{V}_{l}\left(\mathrm{H}^{1}\left(\mathcal{C}_{\overline{\mathbb{F}}_{q}}, \mathcal{A}\right)\right) \stackrel{\sim}{\longrightarrow} \mathrm{V}_{l}\left(\operatorname{Br}\left(\mathcal{S}_{\overline{\mathbb{F}}_{q}}\right)\right)
$$

The result follows from this.

\subsection{Comparison and criteria. Define}

$$
\chi\left(\mathcal{S}, \mathcal{O}_{\mathcal{S}}\right)=\sum_{n}(-1)^{n} \operatorname{dim}_{\mathbb{F}_{q}} \mathrm{H}^{n}\left(\mathcal{S}, \mathcal{O}_{\mathcal{S}}\right)
$$

By [Liu et al. 2004, Equation (14)], we have

$$
\chi\left(\mathcal{S}, \mathcal{O}_{\mathcal{S}}\right)=\operatorname{deg}(\mathcal{L})+(1-\operatorname{dim}(A))\left(1-g_{\mathcal{C}}\right)
$$


By Milne's formula [1975, the last equation of Section 6] on Euler characteristics or Crew's formula [Illusie 1983, Proposition 6.3.9] on Hodge-Witt numbers, we have

$$
\sum_{\lambda_{\mathcal{S}, j}<1}\left(1-\lambda_{\mathcal{S}, j}\right)=\chi\left(\mathcal{S}, \mathcal{O}_{\mathcal{S}}\right)-\left(1-\operatorname{dim} \operatorname{Pic}_{\mathcal{S} / \mathbb{F}_{q}, \text { red }}^{0}+s_{\mathcal{S}}\right) .
$$

Combining these with Propositions 3.1.5 and 3.2.1, we have:

Corollary 3.4.1. The formula (14) is equivalent to the formula (10) for the Jacobian A. In particular, the formula (10) holds for A without any assumption on $\amalg$ (or the reduction type of A). In particular, it holds for any elliptic curve $A / K$.

The equivalence between Milne's formula for $\mathcal{S}$ and the formula (10) for a Jacobian $A$ is suggested by Ulmer [2019, Remark 4.3(3,4)]. The above corollary verifies his suggestion.

We will give some criteria and cases of $\mu=0$ and $\mu>0$.

Proposition 3.4.2. The following are equivalent:

(i) $\mu_{A / K}=0$.

(ii) $\mathrm{H}_{\mathrm{fl}}^{2}\left(\mathcal{S}_{\overline{\mathbb{F}}_{q}}, \mu_{p}\right)$ is finite.

(iii) $\operatorname{Br}\left(\mathcal{S}_{\overline{\mathbb{F}}_{q}}\right)[p]$ is finite.

(iv) $\mathcal{S}$ is of Hodge-Witt type, i.e., $\mathrm{H}^{i}\left(\mathcal{S}, W \Omega_{\mathcal{S}}^{j}\right)$ is a finitely generated $W\left(\mathbb{F}_{q}\right)$-module for any $i, j$.

Proof. Let $W=W\left(\mathbb{F}_{q}\right)$. From the definition of $s_{\mathcal{S}}$, we know that $\mu_{A / K}=s_{\mathcal{S}}=0$ if and only if $\underline{\mathrm{H}}^{2}\left(\mu_{p^{\infty}}\right)$ is étale. As $\underline{\mathrm{U}}^{2}\left(\mu_{p^{\infty}}\right)$ is always unipotent, this is equivalent to $\underline{\mathrm{H}}^{2}\left(\mu_{p^{\infty}}\right)[p]$ being étale. The group scheme $\underline{\mathrm{H}}^{1}\left(\mu_{p^{\infty}}\right)$ is always étale [Artin 1974b, the paragraph after Theorem (3.1); Milne 1976, Theorem 5.2]. Hence the exact sequence

$$
0 \rightarrow \underline{\mathrm{H}}^{1}\left(\mu_{p^{\infty}}\right) / p \underline{\mathrm{H}}^{1}\left(\mu_{p^{\infty}}\right) \rightarrow \underline{\mathrm{H}}^{2}\left(\mu_{p}\right) \rightarrow \underline{\mathrm{H}}^{2}\left(\mu_{p^{\infty}}\right)[p] \rightarrow 0
$$

shows that $\underline{\mathrm{H}}^{2}\left(\mu_{p^{\infty}}\right)[p]$ is étale if and only if $\underline{\mathrm{H}}^{2}\left(\mu_{p}\right)$ is étale. Since $\underline{\mathrm{H}}^{2}\left(\mu_{p}\right)$ is the perfection of a smooth algebraic group, this is equivalent to its group of geometric points $\mathrm{H}_{\mathrm{fl}}^{2}\left(\mathcal{S}_{\overline{\mathbb{F}}_{q}}, \mu_{p}\right)$ being finite. The group $\operatorname{Pic}\left(\mathcal{S}_{\overline{\mathbb{F}}_{q}}\right) / p \operatorname{Pic}\left(\mathcal{S}_{\overline{\mathbb{F}}_{q}}\right)$ is always finite. Hence $\mathrm{H}_{\mathrm{fl}}^{2}\left(\mathcal{S}_{\overline{\mathbb{F}}_{q}}, \mu_{p}\right)$ is finite if and only if $\operatorname{Br}\left(\mathcal{S}_{\overline{\mathbb{F}}_{q}}\right)[p]$ is finite. This proves the first three equivalences.

For the equivalence of (i) and (iv), first recall that $\mathrm{H}^{2}\left(\mathcal{S}, W \mathcal{O}_{\mathcal{S}}\right)$ is finitely generated over $W \llbracket V \rrbracket$, its $p$ primary torsion submodule $\mathrm{H}^{2}\left(\mathcal{S}, W \mathcal{O}_{\mathcal{S}}\right)\left[p^{\infty}\right]$ is killed by a power of $p$, and the quotient of $\mathrm{H}^{2}\left(\mathcal{S}, W \mathcal{O}_{\mathcal{S}}\right)$ by $\mathrm{H}^{2}\left(\mathcal{S}, W \mathcal{O}_{\mathcal{S}}\right)\left[p^{\infty}\right]$ is finite free over $W$ by [Illusie 1979, II, corollaire 2.11, théorème 2.13]. In particular, $\mu_{A / K}=s_{\mathcal{S}}=0$ if and only if $\mathrm{H}^{2}\left(\mathcal{S}, W \mathcal{O}_{\mathcal{S}}\right)\left[p^{\infty}\right] \otimes_{W \llbracket V \rrbracket} W((V))$ has length 0 as $W((V))$-module, i.e., is trivial.

To prove the equivalence, first assume that $\mathrm{H}^{2}\left(\mathcal{S}, W \mathcal{O}_{\mathcal{S}}\right)\left[p^{\infty}\right] \otimes_{W \llbracket V \rrbracket} W((V))$ is zero. This implies that $\mathrm{H}^{2}\left(\mathcal{S}, W \mathcal{O}_{\mathcal{S}}\right)[p] \otimes_{W \llbracket V \rrbracket} W((V))$, or $\mathrm{H}^{2}\left(\mathcal{S}, W \mathcal{O}_{\mathcal{S}}\right)[p] \otimes_{k \llbracket V \rrbracket} k((V))$ is zero. Since $\mathrm{H}^{2}\left(\mathcal{S}, W \mathcal{O}_{\mathcal{S}}\right)[p]$ is finitely generated over $k \llbracket V \rrbracket$, this implies that $\mathrm{H}^{2}\left(\mathcal{S}, W \mathcal{O}_{\mathcal{S}}\right)[p]$ is finite-dimensional over $k$. Then $\mathrm{H}^{2}\left(\mathcal{S}, W \mathcal{O}_{\mathcal{S}}\right)\left[p^{\infty}\right]$ has finite length over $W$. Hence $\mathrm{H}^{2}\left(\mathcal{S}, W \mathcal{O}_{\mathcal{S}}\right)$ is finitely generated over $W$. Since $\mathcal{S}$ 
is a surface, by Nygaard's theorem [Illusie 1979, II, corollaire 3.14], we know that the slope spectral sequence for $\mathcal{S}$ degenerates at $E_{1}$. Since crystalline cohomology is finitely generated over $W$, this implies that all the $E_{1}$-terms are finitely generated over $W$. Hence $\mathcal{S}$ is of Hodge-Witt type.

Conversely, assume that $\mathcal{S}$ is of Hodge-Witt type. Then $\mathrm{H}^{2}\left(\mathcal{S}, W \mathcal{O}_{\mathcal{S}}\right)\left[p^{\infty}\right]$ has finite length over $W$. Since the $V$-action is topologically nilpotent, this implies that $\mathrm{H}^{2}\left(\mathcal{S}, W \mathcal{O}_{\mathcal{S}}\right)\left[p^{\infty}\right]$ is killed by a power of $V$. This proves the claim.

Remark 3.4.3. It is a nontrivial result [Illusie and Raynaud 1983, III, théorème (4.13)] that if $\mathcal{S}$ is ordinary in the sense of Illusie and Raynaud [1983, IV, définition (4.12)], then it is of Hodge-Witt type. The reciprocal statement does not hold however.

Proposition 3.4.4. Assume that $\mathcal{S}$ is relatively minimal over $\mathcal{C}$ and has Kodaira dimension $\leq 0$. Then $\mu_{A / K} \neq 0$ if and only if $\mu_{A / K}=1$ if and only if $\mathcal{S}$ is a supersingular abelian surface or a supersingular K3 surface.

Proof. We will use the Kodaira-Enriques classification [Liedtke 2013, §§6, 7]. If the Kodaira dimension of $\mathcal{S}$ is $-\infty$, then $\mathcal{S}$ is of Hodge-Witt type by [Joshi 2020, $§ 5.8$, Proposition 5.6], so $\mu_{A / K}=0$. If $\mathcal{S}$ is an abelian surface or a K3 surface, then it is not of Hodge-Witt type if and only if it is supersingular by [Joshi 2020, Proposition 5.9], in which case the formal Brauer group of $\mathcal{S}$ is the formal completion of the additive group by [Illusie 1979, II, $\S \S 7.1,7.2$ ] and hence $\mu_{A / K}=1$ by Remark 3.1.4. If $\mathcal{S}$ is (quasi)hyperelliptic, then $\operatorname{dim} \operatorname{Pic}_{\mathcal{S} / \mathbb{F}_{q} \text {,red }}^{0}=1$ and $\chi\left(\mathcal{S}, \mathcal{O}_{\mathcal{S}}\right)=0$ by the table in Introduction of [Bombieri and Mumford 1977] (with $\operatorname{Pic}_{\mathcal{S} / \mathbb{F}_{q}}^{0}$ reduced or not). Hence by [Suwa 1983, Corollary 5], we know that $\mathcal{S}$ is of Hodge-Witt type. If $\mathcal{S}$ is Enriques, then it is of Hodge-Witt type by [Illusie 1979, II, corollaire 7.3.3(a)].

An explicit example is given as follows.

Proposition 3.4.5. Suppose $p \equiv 3 \bmod 4$. Let $\mathcal{S} \rightarrow \mathcal{C}=\mathbb{P}_{\mathbb{F}_{q}}^{1}$ be Shioda's modular elliptic surface of level 4 [Shioda 1972, Appendices A-C; 1975, §3] (see also [Shimada 2020]), whose generic fiber $\mathcal{S}_{K}=A$ is the elliptic curve defined by the equation

$$
y^{2}=x(x-1)\left(x-\frac{1}{4}\left(t+\frac{1}{t}\right)^{2}\right),
$$

where $t$ is the coordinate of $\mathbb{P}_{\mathbb{F}_{q}}^{1}$. Then $\mu_{A / K}=1$, and A has semistable reduction everywhere.

Proof. By [Shioda 1972, Appendix A(viii); 1975, §3, Theorem 1, Corollary 1] (see also [Shimada 2020, Theorem 1.1(1,2)]), we know that $\mathcal{S}$ is a supersingular K3 surface semistable everywhere over $\mathcal{C}$. Hence this proposition is a special case of the previous proposition.

Little can be said systematically about the case of positive Kodaira dimension, except Proposition 5.1.2, Section 6 and an example in Section 7.3 below.

Remark 3.4.6. (1) In the proof of Corollary 3.4.1, we saw that Crew's formula on Hodge-Witt numbers of $\mathcal{S}$ (with $i=0$ ) is equivalent to the $\mu$-invariant formula (10) for the Jacobian $A / K$. By [Illusie 1983, (6.3.5-8)], Crew's formula is applicable to any object of $D_{c}^{b}(R)$, where $R$ is the Raynaud ring. We used this 
formula for the de Rham-Witt cohomology $R \Gamma\left(\mathcal{S}, W \Omega_{\mathcal{S}}\right) \in D_{c}^{b}(R)$. On the other hand, Illusie [1983, at the end] expressed his hope to define a theory of de Rham-Witt complexes with coefficients in $F$-crystals.

With these observations in mind, one may speculate that there should be a "de Rham-Witt complex with coefficients in the Néron model $\mathcal{A}$," "W $\Omega_{\mathcal{C}} \otimes_{W \mathcal{O}_{\mathcal{C}}} D(\mathcal{A})$," corresponding to an arbitrary abelian variety $A / K$, so that Crew's formula for $R \Gamma\left(\mathcal{C}, W \Omega_{\mathcal{C}} \otimes_{W \mathcal{O}_{\mathcal{C}}} D(\mathcal{A})\right) \in D_{c}^{b}(R)$ contains the $\mu$-invariant formula (10) for $A / K$ as a special case.

(2) Let $\mathcal{S}$ and $A=\operatorname{Pic}_{\mathcal{S}_{K} / K}^{0}$ as above. Consider the canonical domino associated with the graded $R$-module $\mathrm{H}^{2}\left(\mathcal{S}, W \mathcal{O}_{\mathcal{S}}\right) \rightarrow \mathrm{H}^{2}\left(\mathcal{S}, W \Omega_{\mathcal{S}}^{1}\right)$ as in [Illusie 1983, Proposition 2.5.2(ii)]. It admits a finite filtration whose successive subquotients are isomorphic to elementary dominoes $\underline{\underline{U}}_{i_{1}}, \ldots, \underline{\underline{U}}_{i_{m}}$ [Illusie 1983, §2.2.2]. The number $m$ of the elementary dominoes is the dimension of this domino in the sense of [Illusie 1983, paragraph after Proposition 2.5.2], which is equal to $\mu_{A / K}$ by Remark 3.1.4, Proposition 3.1.5 and [Illusie $1983, \S 2.5, \mathrm{~b} 2)]$.

As a more precise relation, can we read off the set of integers $i_{1}, \ldots, i_{m}$ from the Iwasawa theory of $A / K$ ? When $\mathcal{S}$ is a supersingular $\mathrm{K} 3$ surface (so $m=1$ ), the number $i=i_{1}=i_{m}$ is called the Artin invariant and usually denoted by $\sigma_{0}$ [Illusie 1983, §2.2.2 b); Artin 1974b, Equation (4.6)], which satisfies the property that the discriminant of the intersection pairing on the geometric Néron-Severi group of $\mathcal{S}$ is $-p^{2 \sigma_{0}}$ (where $p \neq 2$ ). For a more general $\mathcal{S}$, the integers $i_{1}, \ldots, i_{m}$ might be related to the geometric Cassels-Tate discriminant group $\delta_{\mathrm{CT}}$ [Suzuki 2020, Theorem 3.4.1(6g)] and the analogue of Artin's period map [Suzuki 2020, paragraphs after Theorem 3.4.1].

It is an interesting problem to see if the numbers $i_{1}, \ldots, i_{m}$ define a nice stratification on the moduli of elliptic curves with given $\operatorname{deg}(\Delta)$ in Section 6, generalizing Artin's filtration on the moduli of supersingular K3 surfaces [Artin 1974b, §7].

\section{The $\mu$-invariant for semistable abelian varieties}

The aim of this section is to prove that the $\mu$-invariant formula (10) holds for semistable abelian varieties $A$ without any assumption on $\amalg$.

\subsection{Statement and the p-adic L-function.}

Theorem 4.1.1. Let A be a semistable abelian variety over $K$. Then the formula (10) holds for A.

We will prove this below. Let $Q(\Lambda)$ be the fraction field of $\Lambda$. Let $\mathcal{Z}$ be a finite set of places of $K$ such that $A$ has good reduction over $\mathcal{C} \backslash \mathcal{Z}$. View the Frobenius element $\operatorname{Frob}_{q} \in \operatorname{Gal}\left(\mathbb{F}_{q, \infty} / \mathbb{F}_{q}\right)$ sending $x$ to $x^{q}$ as an element in $\Gamma$. For each place $v$, set $\operatorname{Frob}_{v}=\operatorname{Frob}_{q}^{\operatorname{deg}(v)}$ and $q_{v}=q^{\operatorname{deg}(v)}$. Let $P_{v}(t)$ be the Euler factor at $v$ of the $L$-function of $A$, which is a polynomial in $t=q^{-s}$ with $\mathbb{Z}$-coefficients and constant term 1. Let $L_{A, \mathcal{Z}}(s)$ be the $L$-function of $A$ without the Euler factors at points of $\mathcal{Z}$, so that

$$
L_{A, \mathcal{Z}}(s)=L_{A}(s) \cdot \prod_{v \in \mathcal{Z}} P_{v}\left(q_{v}^{-s}\right)=\frac{P_{1}\left(q^{-s}\right)}{P_{0}\left(q^{-s}\right) P_{2}\left(q^{-s}\right)} \prod_{v \in \mathcal{Z}} P_{v}\left(q_{v}^{-s}\right) .
$$


Put

$$
\mathcal{L}_{A / K_{\infty}^{(p)}}:=\frac{P_{1}\left(q^{-1} \operatorname{Frob}_{q}^{-1}\right)}{P_{0}\left(q^{-1} \operatorname{Frob}_{q}^{-1}\right) P_{2}\left(q^{-1} \operatorname{Frob}_{q}^{-1}\right)} \prod_{v \in \mathcal{Z}} P_{v}\left(q_{v}^{-1} \operatorname{Frob}_{v}^{-1}\right)
$$

and regard it as an element in $Q(\Lambda)$. It is actually a rational function of the variable Frob with $_{\mathbb{Q}}$ coefficients (i.e., an element of the rational function field $\mathbb{Q}\left(\right.$ Frob $\left._{q}\right)$ ) and the substitution Frob $_{q}^{-1} \mapsto q^{-s}$ turns it into $L_{A, \mathcal{Z}}(s+1)$.

Proposition 4.1.2. The element $\mathcal{L}_{A / K_{\infty}^{(p)}}$ coincides with the p-adic L-function of [Lai et al. 2016].

Proof. By [Lai et al. 2016, §3.1.2], the $p$-adic $L$-function is defined as

$$
\prod_{i=0}^{2} \operatorname{det}_{\Lambda[1 / p]}\left(1-\Phi_{0}^{i} \otimes\left[\operatorname{Frob}_{q}^{-1}\right], P_{0}^{i} \otimes_{\mathbb{Z}_{p}} \Lambda[1 / p]\right)^{(-1)^{i+1}},
$$

where $P_{0}^{i}:=\mathrm{H}_{\text {log-crys, } c}^{i}\left(\mathcal{C}^{\sharp} / \mathbb{Z}_{p}, D(A)\right)$ endowed with its Frobenius operator $\Phi_{0}^{i}$ (Here $D(A)$ is the $\log$ Dieudonné crystal associated with the semistable abelian variety $A$ following [Kato and Trihan 2003] and $\mathcal{C}^{\sharp}$ is the log-scheme with underlying scheme $\mathcal{C}$ and log-structure induced by $\mathcal{Z}$ ). On the other hand, by [Kato and Trihan 2003, §3.2.1], the right-hand side of (15) is

$$
\prod_{i=0}^{2} \operatorname{det}_{\mathbb{Q}_{p}}\left(1-\operatorname{Frob}_{q}^{-1} \Phi_{0}^{i}, P_{0}^{i} \otimes \mathbb{Q}_{p}\right)^{(-1)^{i+1}} .
$$

Then by [Lai et al. 2016, Theorem 1.1(2)], for a characteristic element $c_{A / K_{\infty}^{(p)}}$ of $X_{A / K}$,

$$
\mathcal{L}_{A / K_{\infty}^{(p)}}=u \cdot \star \cdot c_{A / K_{\infty}^{(p)}},
$$

where $u \in \Lambda^{\times}$, and $\star$ is given as follow. For each $v \in \mathcal{Z}$, let $T_{v}$ be the maximal torus of $\mathcal{A}_{v}^{0}$ and set $B_{v}=\mathcal{A}_{v}^{0} / T_{v}$ (which is an abelian variety over $k(v)$ since $A$ is semistable). Let $g_{v}=\operatorname{dim}\left(B_{v}\right)$. Let $\left\{\beta_{i}^{(v)}\right\}$ be the eigenvalues of Frob $v$ on the $l$-adic Tate module of $B_{v}$ (for any $l \neq p$ ) viewed as elements of $\overline{\mathbb{Q}}$ and hence $\overline{\mathbb{Q}}_{p}$. (Here, as usual, the Tate module $\mathrm{T}_{l}\left(B_{v}\right)$ means $\mathrm{T}_{l}\left(B_{v}\left(\overline{\mathbb{F}}_{q}\right)\right)$ with the natural action of the $q_{v}$-th power Frobenius Frob $_{v}$.)

Let $\zeta_{1}, \ldots, \zeta_{m}$ be the eigenvalues of Frob $_{q}$ on the $p$-adic Tate module of $A\left(K_{\infty}^{(p)}\right)$. Then

$$
\star=\frac{q^{-\operatorname{dim}(A)\left(\operatorname{deg}(\mathcal{Z})+g_{\mathcal{C}}-1\right)-\operatorname{deg}(\mathcal{L})} \cdot \prod_{v \in \mathcal{Z}} \prod_{i=1}^{2 g(v)}\left(\beta_{i}^{(v)}-\text { Frob }_{v}^{-1}\right)}{\prod_{i=1}^{m}\left(1-\zeta_{i}^{-1} \operatorname{Frob}_{q}\right)\left(1-\zeta_{i}^{-1} \operatorname{Frob}_{q}^{-1}\right)} .
$$

(The term $\delta$ in the original formula is equal to $\operatorname{deg}(\mathcal{L})$ here by [Geisser and Suzuki 2020, Proposition 3.3] for example.)

4.2. Local factors and the trace. To prove the theorem, we will simplify both sides of (17). For each $v \in \mathcal{Z}$, let $t(v)=\operatorname{dim}\left(T_{v}\right)$, so that $t(v)+g(v)=\operatorname{dim}(A)$. Let $P_{v \text {, tor }}(t)$ (resp. $\left.P_{v, \text { ab }}(t)\right)$ be the characteristic polynomial of $q_{v}$ Frob $_{v}^{-1}$ on $\mathrm{V}_{l}\left(T_{v}\right)\left(\operatorname{resp} . \mathrm{V}_{l}\left(B_{v}\right)\right)$, so that $P_{v}(t)=P_{v \text {, tor }}(t) P_{v \text {, ab }}(t)$. We denote by $\equiv$ the equality in $Q\left(\Lambda \otimes_{\mathbb{Z}_{p}} \mathcal{O}\right)^{\times} /\left(\Lambda \otimes_{\mathbb{Z}_{p}} \mathcal{O}\right)^{\times}$, where $\mathcal{O}$ is the ring of integers of some large enough finite extension of $\mathbb{Q}_{p}$. Note that $Q(\Lambda)^{\times} / \Lambda^{\times}$injects into $Q\left(\Lambda \otimes_{\mathbb{Z}_{p}} \mathcal{O}\right)^{\times} /\left(\Lambda \otimes_{\mathbb{Z}_{p}} \mathcal{O}\right)^{\times}$. 
Lemma 4.2.1. For any $v \in \mathcal{Z}$, we have

$$
\begin{gathered}
P_{v, \text { tor }}\left(q_{v}^{-1} \operatorname{Frob}_{v}^{-1}\right) \equiv q_{v}^{-t(v)}, \quad P_{v, \mathrm{ab}}\left(q_{v}^{-1} \operatorname{Frob}_{v}^{-1}\right) \equiv q_{v}^{-g(v)} \prod_{i=1}^{2 g(v)}\left(\beta_{i}^{(v)}-\operatorname{Frob}_{v}^{-1}\right), \\
P_{0}\left(q^{-1} \operatorname{Frob}_{q}^{-1}\right) \equiv q^{-\operatorname{dim} \operatorname{Tr}_{K / \mathbb{F}_{q}}(A)} \cdot \prod_{i=1}^{m}\left(1-\zeta_{i}^{-1} \operatorname{Frob}_{q}^{-1}\right) \\
P_{2}\left(q^{-1} \operatorname{Frob}_{q}^{-1}\right) \equiv \prod_{i=1}^{m}\left(1-\zeta_{i}^{-1} \operatorname{Frob}_{q}\right),
\end{gathered}
$$

Proof. For $P_{v \text {,tor }}$, let $\left\{\gamma_{j}^{(v)}\right\}$ be the eigenvalues of $\operatorname{Frob}_{v}$ on $\mathrm{V}_{l}\left(T_{v}\right)$. Then $q_{v}^{-1} \gamma_{j}^{(v)}$ are roots of unity. We have

$$
P_{v, \text { tor }}\left(q_{v}^{-1} \operatorname{Frob}_{v}^{-1}\right) \prod_{j}\left(1-\left(\gamma_{j}^{(v)}\right)^{-1} \operatorname{Frob}_{v}^{-1}\right)=q_{v}^{-t(v)} \prod_{j}\left(q_{v}-q_{v}\left(\gamma_{j}^{(v)}\right)^{-1} \operatorname{Frob}_{v}^{-1}\right) .
$$

Each factor $q_{v}-q_{v}\left(\gamma_{j}^{(v)}\right)^{-1} \operatorname{Frob}_{v}^{-1}$ is a unit in $\Lambda \otimes_{\mathbb{Z}_{q}} \mathcal{O}$ for some $\mathcal{O}$.

For $P_{v, \text { ab }}$, we have

$$
P_{v, \mathrm{ab}}\left(q_{v}^{-1} \operatorname{Frob}_{v}^{-1}\right)=\prod_{i}\left(1-\left(\beta_{i}^{(v)}\right)^{-1} \operatorname{Frob}_{v}^{-1}\right)=\prod_{i}\left(\beta_{i}^{(v)}\right)^{-1} \prod_{i}\left(\beta_{i}^{(v)}-\operatorname{Frob}_{v}^{-1}\right) .
$$

The product $\prod_{i}\left(\beta_{i}^{(v)}\right)$ is a Weil $q_{v}$-number of weight $2 g(v)$ that is in $\mathbb{Z}$. Hence it is $\pm q_{v}^{g(v)}$.

For $P_{0}$, first notice that by the Lang-Néron theorem [Conrad 2006, Theorem 7.1], the group

$$
A\left(K_{\infty}^{(p)}\right) / \operatorname{Tr}_{K / \mathbb{F}_{q}}(A)\left(\mathbb{F}_{q, \infty}\right)
$$

is a finitely generated abelian group. Therefore the $p$-adic Tate module of $\operatorname{Tr}_{K / \mathbb{F}_{q}}(A)\left(\mathbb{F}_{q, \infty}\right)$ injects into that of $A\left(K_{\infty}^{(p)}\right)$ with finite cokernel. Hence $\zeta_{i}$ are the eigenvalues of Frob $_{q}$ on the $p$-adic Tate module of $\operatorname{Tr}_{K / \mathbb{F}_{q}}(A)\left(\mathbb{F}_{q, \infty}\right)$. In particular, they are $p$-adic units. Let $g_{0}=\operatorname{dim} \operatorname{Tr}_{K / \mathbb{F}_{q}}(A)$.

By [Tan 2014, Proposition 2.9] and the proof of [Lai et al. 2016, Lemma 2.2.1], we know that $\zeta_{1}, \ldots, \zeta_{m}$ are part of the eigenvalues $\zeta_{1}, \ldots, \zeta_{m}, \ldots, \zeta_{2 g_{0}}$ of $\operatorname{Frob}_{q}$ on the $l$-adic Tate module of $\operatorname{Tr}_{K / \mathbb{F}_{q}}(A)$ (for any $l \neq p)$ and

$$
\prod_{i=1}^{m}\left(\zeta_{i}-\operatorname{Frob}_{q}^{-1}\right) \equiv \prod_{i=1}^{2 g_{0}}\left(\zeta_{i}-\operatorname{Frob}_{q}^{-1}\right) .
$$

Together with the relation between $P_{0}$ and $\operatorname{Tr}_{K / \mathbb{F}_{q}}(A)$ we saw in Section 2.2, we have

$$
\begin{aligned}
P_{0}\left(q^{-1} \operatorname{Frob}_{q}^{-1}\right)=\prod_{i=1}^{2 g_{0}}\left(1-\zeta_{i}^{-1} \operatorname{Frob}_{q}^{-1}\right) & \equiv q^{-g_{0}} \prod_{i=1}^{2 g_{0}}\left(\zeta_{i}-\operatorname{Frob}_{q}^{-1}\right) \\
& \equiv q^{-g_{0}} \prod_{i=1}^{m}\left(\zeta_{i}-\operatorname{Frob}_{q}^{-1}\right) \equiv q^{-g_{0}} \prod_{i=1}^{m}\left(1-\zeta_{i}^{-1} \operatorname{Frob}_{q}^{-1}\right) .
\end{aligned}
$$


For $P_{2}$, first notice that (18) also holds with Frob $_{q}$ replaced by Frob $_{q}^{-1}$ since Frob $_{q} \leftrightarrow$ Frob $_{q}^{-1}$ is an automorphism of $\Lambda$. Also, $\left\{\zeta_{i}\right\}_{i=1}^{2 g_{0}}=\left\{q / \zeta_{i}\right\}_{i=1}^{2 g_{0}}$ (counting the multiplicities) by the functional equation for the zeta function of $\operatorname{Tr}_{K / \mathbb{F}_{q}}(A)$. Using these, we have

$$
\begin{aligned}
P_{2}\left(q^{-1} \operatorname{Frob}_{q}^{-1}\right) & =\prod_{i=1}^{2 g_{0}}\left(1-q \zeta_{i}^{-1} \operatorname{Frob}_{q}^{-1}\right)=\prod_{i=1}^{2 g_{0}}\left(1-\zeta_{i} \operatorname{Frob}_{q}^{-1}\right) \\
& \equiv \prod_{i=1}^{2 g_{0}}\left(\zeta_{i}-\operatorname{Frob}_{q}\right) \equiv \prod_{i=1}^{m}\left(\zeta_{i}-\operatorname{Frob}_{q}\right) \equiv \prod_{i=1}^{m}\left(1-\zeta_{i}^{-1} \operatorname{Frob}_{q}\right) .
\end{aligned}
$$

Using this lemma, we see that (17) is equivalent to

$$
c_{A / K_{\infty}^{(p)}} \equiv q^{\operatorname{deg}(\mathcal{L})+\operatorname{dim}(A)\left(g_{\mathcal{C}}-1\right)+\operatorname{dim} \operatorname{Tr}_{K / \mathbb{F} q}(A)} \cdot P_{1}\left(q^{-1} \operatorname{Frob}_{q}^{-1}\right) .
$$

The exact $p$-power factor of $c_{A / K_{\infty}^{(p)}}$ is $q^{\mu_{A / K}}$ by definition. The exact $p$-power factor of $P_{1}\left(q^{-1} \operatorname{Frob}_{q}^{-1}\right)$ is $q^{-\theta_{A}}$ by definition. Comparing the exact $p$-power factors of both sides, we obtain Theorem 4.1.1.

\section{The $\mu$-invariant for elliptic curves}

For the rest of the paper, we deal with the case where $\operatorname{dim} A=1$. Let $\Delta:=\sum_{v \in \mathcal{C}} \delta_{v} \cdot v$ the global discriminant of $A / K$, where $\delta_{v}$ is the valuation of the minimal discriminant of $A$ at $v$ [Silverman 1986, VII.1].

When it becomes necessary to emphasize the roles played by $A$ and $K$, we shall use $\Delta_{A / K}$ to denote it. By [Conrad 2006, Example 2.2], we know $\operatorname{Tr}_{K / \mathbb{F}_{q}}(A)=0$ unless $A$ is constant, in which case $\operatorname{Tr}_{K} / \mathbb{F}_{q}(A)=A$ [Conrad 2006, Theorem 6.4(1)]. In the case $A$ is nonconstant, we have $P_{0}(t)=P_{2}(t)=1$, so we simply write $P(t)=P_{1}(t)$.

5.1. The $\mu$-invariant formula simplified. We have $\operatorname{deg}(\mathcal{L})=\operatorname{deg}(\Delta) / 12$ by [Lai et al. 2016, $\$ 2.2 .1$, Footnote 1]. Therefore the formula (10) (true for $A$ by Corollary 3.4.1) and the inequality (11) become the following statement.

$$
\text { Theorem 5.1.1. } \quad \mu_{A / K}=\frac{\operatorname{deg}(\Delta)}{12}+g_{\mathcal{C}}-1-\theta \leq \frac{\operatorname{deg}(\Delta)}{12}+g_{\mathcal{C}}-1
$$

if $A$ is nonconstant and

$$
\mu_{A / K}=g_{\mathcal{C}}-\theta \leq g_{\mathcal{C}}
$$

if $A$ is constant.

Let $\pi: \mathcal{S} \rightarrow \mathcal{C}$ be the minimal elliptic surface associated with $A / K$. By [Friedman 1998, Chapter 7, Lemma 14] (adapted to positive characteristic),

$$
\operatorname{dim} H^{1}\left(\mathcal{S}, \mathcal{O}_{\mathcal{S}}\right)=g_{\mathcal{C}}, \quad \operatorname{dim} H^{2}\left(\mathcal{S}, \mathcal{O}_{\mathcal{S}}\right)=\operatorname{deg}(\mathcal{L})+g_{\mathcal{C}}-1
$$

if $\mathcal{L}$ is nontrivial, and

$$
\operatorname{dim} H^{1}\left(\mathcal{S}, \mathcal{O}_{\mathcal{S}}\right)=g_{\mathcal{C}}+1, \quad \operatorname{dim} H^{2}\left(\mathcal{S}, \mathcal{O}_{\mathcal{S}}\right)=g_{\mathcal{C}}
$$


if $\mathcal{L}$ is trivial. The canonical bundle formula $\Omega_{\mathcal{S}}^{2} \cong \pi^{*}\left(\Omega_{\mathcal{C}}^{1} \otimes \mathcal{L}\right)$ implies that the Kodaira dimension of $\mathcal{S}$ is $-\infty, 0,1$ if $\operatorname{deg}(\mathcal{L})+2 g_{\mathcal{C}}-2<0, \operatorname{deg}(\mathcal{L})+2 g_{\mathcal{C}}-2=0, \operatorname{deg}(\mathcal{L})+2 g_{\mathcal{C}}-2>0$, respectively (see for instance [Ulmer 2011, Lecture 3, Proposition 4.3] and the paragraphs after $\left.{ }^{6}\right)$.

Let $\bar{r}$ be the geometric Mordell-Weil rank of $A$, i.e., the rank of $A\left(K \overline{\mathbb{F}}_{q}\right) / \operatorname{Tr}_{K / \mathbb{F}_{q}}(A)\left(\overline{\mathbb{F}}_{q}\right)$. Recall from Definition 2.2.2 that $a=a_{A}$ denotes the degree of the polynomial $P_{1}(t)$. Then (13) shows that $\bar{r} \leq a$.

Proposition 5.1.2. Assume that the minimal elliptic surface $\mathcal{S} \rightarrow \mathcal{C}$ over $\mathbb{F}_{q}$ associated with $E / K$ has Kodaira dimension 1 and that $\bar{r}=a$. Then $\mu_{A / K}>0$.

Proof. By the proof of Proposition 3.3.1 and by (12), (13), we know that $a-\bar{r}=\operatorname{deg} P_{\mathcal{S}, 2}-\rho_{\mathcal{S}_{\overline{\mathbb{F}}}}$, where $\rho_{\mathcal{S}_{\overline{\mathbb{F}}}}$ is the rank of $\operatorname{NS}\left(\mathcal{S}_{\overline{\mathbb{F}}_{q}}\right)$. Hence the assumption implies that $\rho_{\mathcal{S}_{\overline{\mathbb{F}}}}=\operatorname{deg} P_{\mathcal{S}, 2}$, i.e., $\mathcal{S}$ is Shioda supersingular. In particular (cf. the paragraph after [Liedtke 2013, Definition 9.11]), it is Artin supersingular, i.e., $\lambda_{\mathcal{S}, i}=1$ for all $i$, or $\theta_{\mathcal{S}}=0$. By Proposition 3.3.1, we know that $\theta_{A}=0$. We have $\operatorname{deg}(\mathcal{L})+2 g_{\mathcal{C}}-2>0$ as above. Hence if $g_{\mathcal{C}}=0$, then $\operatorname{deg}(\mathcal{L}) \geq 3$ and $A$ is nonconstant; if $g_{\mathcal{C}}=1$, then $\operatorname{deg}(\mathcal{L}) \geq 1$ and $A$ is nonconstant; or else $g_{\mathcal{C}} \geq 2$. Applying Theorem 5.1.1, we know $\mu_{A / K}>0$.

5.2. The $j$-invariant. Let $j_{A}$ denote the $j$-invariant of $A$. Recall the Szpiro difference $d=d_{A}$ from Definition 2.5.3.

Theorem 5.2.1 [Pesenti and Szpiro 2000, théorème 0.1]. If $j_{A}$ is not a $p$-th power or $A$ is isotrivial, then $d \geq 0$.

Since $\mu_{A / K}=b-d$ by Corollary 2.5.4, this theorem allows us to bound $\mu_{A / K}$ purely in terms of the $L$-function:

Corollary 5.2.2. If $j_{A}$ is not a $p$-th power or $A$ is isotrivial, then $\mu_{A / K} \leq b$.

For the rest of this section, we further assume that the elliptic curve $A$ is nonisotrivial. We will study the behavior of the $j$-invariant and the $\mu$-invariant under isogeny.

Lemma 5.2.3. The following conditions are equivalent

(a) The group scheme $A_{p}$ has a nontrivial étale subgroup.

(b) The $j$-invariant $j_{A}$ is a p-th power of an element in $K$.

Proof. If $E$ is a nontrivial étale subgroup scheme of $A_{p}$, then it is of order $p$ and $B:=A / E$ is isogenous to $A$ by the natural homomorphism $\varphi: A \rightarrow B$. Because the group scheme $C=\varphi\left(A_{p}\right)$ is connected and hence $B / C=A$ is isomorphic to $B^{(p)}, \varphi$ is isomorphic to the Verschiebung. This shows $j_{A}=j_{B^{(p)}}$ is a $p$-th power. Conversely, if $j_{A}=j_{0}^{p}$ and let $B / K$ be an elliptic curve with $j_{B}=j_{0}$, then $j_{A}=j_{B^{(p)}}$ and hence $A$ and $B^{(p)}$ are isomorphic over the separable closure of $K$ [Silverman 1986, III.1.4(b), A.1.2(b)]. Since $B_{p}^{(p)}$ contains a nontrivial étale subgroup scheme, so does $A$.

\footnotetext{
${ }^{6}$ We take the opportunity to mention a mistake in [Ulmer 2011, Lecture 3(6.3)]. The correct formula can be found in [Shioda 1992, Equation (2.9) and Lemma 6].
} 
Lemma 5.2.4. Every nonisotrivial elliptic curve is isogenous to an elliptic curve with $j$-invariant not a p-th power.

Proof. Let $\mathcal{E}$ be the maximal étale subgroup of $A_{p^{\infty}}$. Then $\mathcal{E}$ is finite and $(A / \mathcal{E})_{p}$ has no nontrivial étale subgroup.

5.3. The isogeny class. Consider the connected-étale sequence

$$
0 \rightarrow A_{p^{n}}^{0} \rightarrow A_{p^{n}} \rightarrow \pi_{0}\left(A_{p^{n}}\right) \rightarrow 0,
$$

where $A_{p^{n}}^{0}$ and $\pi_{0}\left(A_{p^{n}}\right)$ are Cartier dual to each other. Since $\pi_{0}\left(A_{p^{n}}\right)$ is of $p$-rank 1 , there exists a unique filtration

$$
0 \subset G_{1} \subset \cdots \subset G_{n-1} \subset G_{n}=\pi_{0}\left(A_{p^{n}}\right)
$$

with $G_{i}$ of order $p^{i}$, and by duality the filtration

$$
0 \subset H_{1} \subset \cdots \subset H_{n-1} \subset H_{n}=A_{p^{n}}^{0}
$$

with $H_{i}$ of order $p^{i}$. If $\varphi: A \rightarrow B$ is an isogeny of degree $p^{n}$, then $E:=\operatorname{Ker} \varphi \subset A_{p^{n}}$ with $E^{0} \subset A_{p^{n}}^{0}$ and $\pi_{0}(E) \subset \pi_{0}\left(A_{p^{n}}\right)$. By the above filtrations, $\varphi$ is decomposed into a sequence

$$
A \stackrel{\phi_{1}}{\longrightarrow} A_{1} \stackrel{\phi_{2}}{\longrightarrow} \cdots \stackrel{\phi_{c}}{\longrightarrow} A_{c} \stackrel{\psi_{1}}{\longrightarrow} B_{1} \stackrel{\psi_{2}}{\longrightarrow} \cdots \stackrel{\psi_{e}}{\longrightarrow} B_{e} \stackrel{\psi_{e+1}}{\longrightarrow} B
$$

where each $\phi_{i}$ is a Frobenius, $\psi_{j}$ a Verschiebung, all of degree $p$. Suppose both $c$ and $e$ are positive. Then up to isomorphisms, the segment

$$
A_{c-1} \stackrel{\phi_{c}}{\longrightarrow} A_{c} \stackrel{\psi_{1}}{\longrightarrow} B_{1}
$$

is equal to the multiplication by $p$. Hence $\varphi$ can be written as $\varphi^{\prime} \circ[p]$ with $\varphi^{\prime}$ of less degree than $\varphi$. By repeating this procedure, we prove the following lemma.

Lemma 5.3.1. If $\varphi: A \rightarrow B$ is an isogeny of degree $p^{n}$ for some $n$, then either $B=A^{\left(p^{m}\right)}$ or $A=B^{\left(p^{m}\right)}$ for some $m$.

Suppose $A / K$ has semistable reduction everywhere. Then

$$
\operatorname{deg} \Delta_{A^{\left(p^{m}\right)} / K}=p^{m} \operatorname{deg} \Delta_{A / K},
$$

while $N_{A^{\left(p^{m}\right) / K}}=N_{A / K}$ and the Hasse-Weil $L$-function for both curves are the same. Hence Theorem 5.1.1 says

$$
\mu_{A^{\left(p^{m}\right) / K}}=\mu_{A / K}+\left(p^{m}-1\right) \cdot \frac{\operatorname{deg} \Delta_{A / K}}{12} .
$$

Lemma 5.3.2. If $\varphi: B \rightarrow A$ is an isogeny of degree $l$ with $(l, p)=1$, then

$$
\operatorname{deg} \Delta_{B / K}=\operatorname{deg} \Delta_{A / K} .
$$

Hence $\mu_{B / K}=\mu_{A / K}$. 
Proof. Choose an invariant differential $\omega^{\prime}$ of $A / K$ and denote $\omega:=\varphi^{*} \omega^{\prime}$. For each place $v$, let $\omega_{v}$ and $\omega_{v}^{\prime}$ respectively be local Néron differentials of $B$ and $A$. Then we have (see [Tan 1995, §1.2])

$$
\operatorname{deg} \Delta_{B / K}=12 \sum_{v} \operatorname{ord}_{v} \frac{\omega}{\omega_{v}} \cdot \operatorname{deg} v, \quad \text { and } \quad \operatorname{deg} \Delta_{A / K}=12 \sum_{v} \operatorname{ord}_{v} \frac{\omega^{\prime}}{\omega_{v}^{\prime}} \cdot \operatorname{deg} v .
$$

Let $\psi: A \rightarrow B$ denote the dual isogeny. Write $\varphi^{*} \omega_{v}^{\prime}=\alpha_{v} \cdot \omega_{v}, \psi^{*} \omega_{v}=\beta_{v} \cdot \omega_{v}^{\prime}, \alpha_{v}, \beta_{v} \in \mathcal{O}_{v}$. But, since $\psi \circ \varphi=[l]$, we have $\alpha_{v} \cdot \beta_{v}=l$. Hence $\alpha_{v} \in \mathcal{O}_{v}^{*}$. Consequently,

so $\operatorname{deg} \Delta_{B / K}=\operatorname{deg} \Delta_{A / K}$.

$$
\operatorname{ord}_{v} \frac{\omega^{\prime}}{\omega_{v}^{\prime}}=\operatorname{ord}_{v} \frac{\varphi^{*} \omega^{\prime}}{\varphi^{*} \omega_{v}^{\prime}}=\operatorname{ord}_{v} \frac{\omega}{\alpha_{v} \cdot \omega_{v}}=\operatorname{ord}_{v} \frac{\omega}{\omega_{v}}
$$

We summarize the above discussion below.

Proposition 5.3.3. Among an isogeny class of elliptic curves with semistable reduction everywhere, those curves with $j$-invariant not a $p$-th power have minimal $\mu$-invariant.

The elliptic curve $A$ in Proposition 3.4.5 has $j$-invariant not a $p$-th power. Hence by Proposition 5.3.3, we cannot replace $A$ by an isogenous elliptic curve for which the $\mu$-invariant is zero. This should be compared with Greenberg's conjecture [1999, Conjecture 1.11] on $\mu$-invariants of elliptic curves over $\mathbb{Q}$. To recall this conjecture, let $E$ be an elliptic curve over $\mathbb{Q}$. Assume that $\operatorname{Sel}_{p}\left(E / \mathbb{Q}^{\text {cyc }}\right)^{\vee}$ is torsion over $\mathbb{Z}_{p} \llbracket \mathrm{Gal}\left(\mathbb{Q}^{\mathrm{cyc}} / \mathbb{Q}\right) \rrbracket$. Then Greenberg's conjecture claims the existence of a $\mathbb{Q}$-isogenous elliptic curve $E^{\prime}$ such that $\operatorname{Sel}_{p^{\infty}}\left(E^{\prime} / \mathbb{Q}^{\mathrm{cyc}}\right)^{\vee}$ has $\mu$-invariant zero.

5.4. Elliptic curves with trivial $\mu$-invariant. In this subsection, we produce infinitely many elliptic curves with trivial $\mu$-invariant.

Assume that $\operatorname{char}(K) \neq 2$ and $A / K$ is defined by the Legendre form (see [Silverman 1986, §III.1.7])

$$
Y^{2}=X(X-1)(X-\lambda),
$$

with $\lambda \in K$, so that $F_{0}:=\mathbb{F}_{q}(\lambda) \subset K$. Equation (21) defines an elliptic curve $E_{0} / F_{0}$ such that $A / K$ is just its base change to $K$. Put $t^{2}=\lambda, F=\mathbb{F}_{q}(t)$ and let $E / F$ be the elliptic curve defined by

$$
Y^{2}=X(X-1)\left(X-t^{2}\right)
$$

Then $E / F$ is the base change of $E_{0}$ to $F$. Let $K^{\prime}=K F$, then $A / K^{\prime}$ is the base change of $E$ to $K^{\prime}$.

Definition 5.4.1. An elliptic curve is of Legendre $p$-type, if:

(1) $\operatorname{char}(K) \neq 2$ and $A$ has the Legendre invariant $\lambda \in K$.

(2) $K / F_{0}$, where $F_{0}=\mathbb{F}_{q}(\lambda)$, is a Galois $p$-extension unramified at supersingular places of $E_{0} / F_{0}$.

Theorem 5.4.2. If $A / K$ is of Legendre $p$-type, then $\mu_{A / K}=0$.

Proof. By Lemma 2.1.2, it is enough to show $\mu_{A / K^{\prime}}=0$. Here $K^{\prime}=K F$ and $F=\mathbb{F}_{q}(t)$ with $t^{2}=\lambda$. Let $E_{0} / F_{0}$ and $E / F$ be the elliptic curves defined by (21) and (22), respectively. By the condition (2), if a place of $F$ ramifies in $K^{\prime}$, then the corresponding place of $F_{0}$ is either an ordinary or additive place for 
$E_{0} / F_{0}$. The only place where $E_{0} / F_{0}$ has additive reduction is where $\lambda=\infty$ (see the proof of Lemma 7.1.1 below). The corresponding place $t=\infty$ of $F$ is an ordinary place for $E / F$ (see Lemma 7.1.1). Therefore $K^{\prime} / F$ is a Galois $p$-extension unramified outside ordinary places of $E / F$. Now Theorem 2.1.5 says we only need to prove $\mu_{E / F}=0$. Lemma 7.1 .1 below says $E / F$ has semistable reduction everywhere, and by simple computation (see Section 7.2), we find $\operatorname{deg}\left(\Delta_{E / F}\right)=12$, and hence $\mu_{E / F}=0$ by Theorem 5.1.1. Thus $\mu_{A / K}=0$.

Remark 5.4.3. Let $A / K$ be an elliptic curve satisfying the condition (1) such that $\mu_{A / K}=0$. The previous theorem is a type of results saying $\mu_{A / L}=0$ for some extension $L / K$ unramified at supersingular places of $A / K$. But $L / K$ being ramified at a supersingular place does not always imply that $\mu_{A / L}>0$. For example, let $K=\mathbb{F}_{3}(t)$ and $A$ defined by $Y^{2}=X(X-1)\left(X-t^{2}\right)$. There is one supersingular place which is the zero of $\left(t^{2}+1\right)$. The extension $L=K(s)$ of $K$ with $s^{2}=1+t^{2}$ is a quadratic extension ramified at $\left(t^{2}+1\right)$. However, Magma gives a trivial $\mu$-invariant for $A / L$ (see Section 7.3 below).

\section{6. $\mu=0$ for generic elliptic curves}

In this section, we show that "generic" elliptic curves over $K$ with $p>3$ in a certain precise sense have trivial $\mu$-invariant (Theorem 6.3.1). This partially extends the result [Artin 1974b, Lemma (5.10)] that the generic fibers of general Weierstrass fibrations which are K3 have $\mu=0$. We do not assume that the elliptic curves are nonisotrivial or semistable.

6.1. An elliptic curve having $\mu=0$ with given $\operatorname{deg}(\Delta)$. We first produce some (isotrivial) elliptic curves having $\mu=0$ with given $\operatorname{deg}(\Delta)$.

Proposition 6.1.1. Assume $p>2$. Let $n$ be a nonnegative integer. Then there exist a finite constant extension $K^{\prime}$ of $K$ and an elliptic curve $E / K^{\prime}$ such that $\operatorname{deg}\left(\Delta_{E / K^{\prime}}\right)=12 n$ and $\mu_{E / K^{\prime}}=0$.

Proof. Let $K^{\prime}$ be a finite constant extension of $K$ having $2 n$ places $v_{1}, \ldots, v_{2 n}$ of degree 1 . We first show that by replacing $K^{\prime}$ by a larger finite constant extension of $K$, we can choose an element $f \in K^{\prime}$ such that $v_{i}(f)$ is odd for any $i$ and $v(f)$ is even for any other place $v$ of $K^{\prime}$. Consider the exact sequence

$$
\left(K \overline{\mathbb{F}}_{q}\right)^{\times} \rightarrow \bigoplus_{v} \mathbb{Z} \rightarrow \operatorname{Pic}\left(\mathcal{C}_{\overline{\mathbb{F}}_{q}}\right) \rightarrow 0
$$

where the middle direct sum is over all places of $K \overline{\mathbb{F}}_{q}$, the first map is the sum of the valuations and the second map is $1 \mapsto v$ for each summand corresponding to a place $v$. Since $\operatorname{Pic}^{0}\left(\mathcal{C}_{\overline{\mathbb{F}}_{q}}\right)$ is the group of geometric points of the Jacobian of $\mathcal{C}$ and hence divisible, this gives an exact sequence

$$
\left(K \overline{\mathbb{F}}_{q}\right)^{\times} \rightarrow \bigoplus_{v} \mathbb{Z} / 2 \mathbb{Z} \rightarrow \mathbb{Z} / 2 \mathbb{Z} \rightarrow 0,
$$

where the second map is the summation map. Consider the element of $\bigoplus_{v} \mathbb{Z} / 2 \mathbb{Z}$ such that the component corresponding to each $v_{i}$ is 1 and the other components are zero. This element belongs to the kernel of the summation map. Hence there exists an element $f \in\left(K \overline{\mathbb{F}}_{q}\right)^{\times}$such that $v_{i}(f) \equiv 1 \bmod 2$ for any $i$ 
and $v(f) \equiv 0 \bmod 2$ for any other $v$. Replace $K^{\prime}$ by a larger finite constant extension of $K$ so that $f$ is in $K^{\prime}$. This choice of $K^{\prime}$ and $f$ does the job.

Let $E_{0}: y^{2}=x^{3}+a x^{2}+b x+c$ be any constant ordinary elliptic curve over $K^{\prime}$. Let its quadratic twist by $f$ be $E: f y^{2}=x^{3}+a x^{2}+b x+c$. This $E$ has $\mu=0$ by [Ochiai and Trihan 2009, Theorem 1.8(1)]. We show $\operatorname{deg}\left(\Delta_{E / K^{\prime}}\right)=12 n$. Let $v$ be any place of $K^{\prime}$. If $v(f)$ is even and hence $f=u \pi^{m}$ for some unit $u$ at $v$, uniformizer $\pi$ at $v$ and integer $m$, then $E$ is isomorphic to $u y^{2}=x^{3}+a x^{2}+b x+c$ over $K_{v}^{\prime}$, which has good reduction at $v$. If $v(f)$ is odd, then similarly, $E$ is isomorphic to $y^{2}=x^{3}+\pi a x^{2}+\pi^{2} b x+\pi^{3} c$ over $K_{v}$ for some uniformizer $\pi$ at $v$. Its discriminant is the discriminant of $E_{0}$ times $\pi^{6}$. Hence this equation is minimal at $v$ and $v\left(\Delta_{E / K^{\prime}}\right)=6$. Thus, by the choice of $f$, we know that $\operatorname{deg}\left(\Delta_{E / K^{\prime}}\right)=6 \cdot 2 n=12 n$.

6.2. Moduli of elliptic curves with given $\operatorname{deg}(\Delta)$. To precisely define "generic" elliptic curves, we need a parameter space for elliptic curves over $K$ with given $\operatorname{deg}(\Delta)$. We follow [Kas 1977]. See also [Miranda 1981; Seiler 1987], which work over positive characteristic as well.

For a perfect field extension $k / \mathbb{F}_{q}$ and an abelian variety $A$ over the fraction field of $K \otimes_{\mathbb{F}_{q}} k$ with Néron model $\mathcal{A}$ over $\mathcal{C}_{k}$, we define $\mu_{A}$ to be the dimension of the group scheme $\boldsymbol{H}^{1}\left(\mathcal{C}_{k}, \mathcal{A}\right)$ over $k$. If $A$ is an elliptic curve and the associated minimal elliptic surface $\mathcal{S}$ has smooth Picard scheme, then this $\mu_{A}$ is equal to the dimension of the unipotent part of the formal Brauer group of $\mathcal{S}$ by Remark 3.1.4 and Proposition 3.1.5 (which are still true with $\mathbb{F}_{q}$ replaced by $k$ by the same proof). When $k=\overline{\mathbb{F}}_{q}$, we denote $\overline{\mathcal{C}}=\mathcal{C}_{\overline{\mathbb{F}}_{q}}$. In what follows, any fiber product is taken over $\overline{\mathbb{F}}_{q}$ when " $x$ " has no specified base. For any integer $n$, let $\operatorname{Pic}_{\overline{\mathcal{C}}_{\overline{\mathcal{F}}}}^{n}$ be the degree $n$ component of the Picard scheme $\operatorname{Pic}_{\overline{\mathcal{C}}_{\overline{\mathbb{F}}} / \overline{\mathbb{F}}_{q}}$, which parametrizes the line bundles of degree $n$. Let $\mathcal{P}$ be a Poincaré sheaf on $\overline{\mathcal{C}} \times \operatorname{Pic}_{\overline{\mathcal{C}} / \overline{\mathbb{F}}_{q}}$ [Kleiman 2005, Exercise 4.3; Bosch et al. 1990, 8.2/4] (rigidified at some closed point of $\overline{\mathcal{C}}$ ). Its restriction to $\overline{\mathcal{C}} \times \operatorname{Pic}_{\overline{\mathcal{C}} / \overline{\mathbb{F}}_{q}}^{n}$ for any $n$ is denoted by $\mathcal{P}_{n}$. Denote the second projection $\overline{\mathcal{C}} \times \operatorname{Pic}_{\overline{\mathcal{C}} / \overline{\mathbb{F}}_{q}}^{n} \rightarrow \operatorname{Pic}_{\overline{\mathcal{C}} / \overline{\mathbb{F}}_{q}}^{n}$ by $\mathrm{pr}_{2}$.

If $n>2 g_{\mathcal{C}}-2$, then for any line bundle $\mathscr{L}$ of degree $n$ on $\overline{\mathcal{C}}$ (that is, any element of $\operatorname{Pic}_{\overline{\mathcal{C}} / \overline{\mathbb{F}}_{q}}\left(\overline{\mathbb{F}}_{q}\right)$ ), the dimension of $\mathrm{H}^{0}(\overline{\mathcal{C}}, \mathscr{L})$ is $n+1-g_{\mathcal{C}}$ by the Riemann-Roch theorem, which does not depend on $\mathscr{L}$. Hence the direct image sheaf $\operatorname{pr}_{2 *} \mathcal{P}_{n}$ on $\operatorname{Pic}_{\overline{\mathcal{C}} / \overline{\mathbb{F}}_{q}}^{n}$ has a constant rank over all points of $\operatorname{Pic}_{\overline{\mathcal{C}}^{\prime} / \overline{\mathbb{F}}_{q}}^{n}$. Therefore Grauert's theorem ([Hartshorne 1977, III, Corollary 12.9]) implies that $\mathrm{pr}_{2 *} \mathcal{P}_{n}$ is a locally free sheaf whose fiber at any point $\mathscr{L}$ of $\operatorname{Pic}_{\overline{\mathcal{C}} / \overline{\mathbb{F}}_{q}}^{n}$ is given by $\mathrm{H}^{0}(\overline{\mathcal{C}}, \mathscr{L})$. Let $\boldsymbol{V}\left(\left(\operatorname{pr}_{2 *} \mathcal{P}_{n}\right)^{\vee}\right) \rightarrow \operatorname{Pic}_{\overline{\mathcal{C}} / \overline{\mathbb{F}}_{q}}^{n}$ be the total space of $\operatorname{pr}_{2 *} \mathcal{P}_{n}$ (where $\vee$ denotes the dual bundle), which is the relative Spec of the symmetric algebra of $\left(\operatorname{pr}_{2 *} \mathcal{P}_{n}\right)^{\vee}$. The closed points of $\boldsymbol{V}\left(\left(\operatorname{pr}_{2 *} \mathcal{P}_{n}\right)^{\vee}\right)$ are given by pairs $(\mathscr{L}, s)$, where $\mathscr{L}$ is a line bundle of degree $n$ on $\overline{\mathcal{C}}$ and $s \in \mathrm{H}^{0}(\overline{\mathcal{C}}, \mathscr{L})$.

Assume $p>3$. Let $n>\left(g_{\mathcal{C}}-1\right) / 2$ be an integer. Let $\mathcal{P}_{n}^{\otimes 4}$ and $\mathcal{P}_{n}^{\otimes 6}$ be the tensor powers of $\mathcal{P}_{n}$, which are line bundles on $\operatorname{Pic}_{\overline{\mathcal{C}} / \mathbb{F}_{q}}^{n}$. Since $6 n \geq 4 n>2 g_{\mathcal{C}}-2$, we have a vector bundle $V\left(\left(\operatorname{pr}_{2 *}\left(\mathcal{P}_{n}^{\otimes 4} \oplus \mathcal{P}_{n}^{\otimes 6}\right)\right)^{\vee}\right)$ over $\operatorname{Pic}_{\overline{\mathcal{C}} / \overline{\mathbb{F}}_{q}}^{n}$ as above. The closed points of $\boldsymbol{V}\left(\left(\operatorname{pr}_{2 *}\left(\mathcal{P}_{n}^{\otimes 4} \oplus \mathcal{P}_{n}^{\otimes 6}\right)\right)^{\vee}\right)$ are given by triples $\left(\mathscr{L}, g_{2}, g_{3}\right)$, where $\mathscr{L}$ is a line bundle of degree $n$ on $\overline{\mathcal{C}}$ and $g_{2} \in \mathrm{H}^{0}\left(\overline{\mathcal{C}}, \mathscr{L}^{\otimes 4}\right), g_{3} \in \mathrm{H}^{0}\left(\overline{\mathcal{C}}, \mathscr{L}^{\otimes 6}\right)$. Following the paragraphs after [Kas 1977, Theorem 1], we make the following definition:

Definition 6.2.1. Define $Y(n, \overline{\mathcal{C}})$ to be the set of triples $\left(\mathscr{L}, g_{2}, g_{3}\right)$ as above satisfying the following two conditions: 
- $4 g_{2}^{3}-27 g_{3}^{2} \neq 0$ as an element of $\mathrm{H}^{0}\left(\overline{\mathcal{C}}, \mathscr{L}^{\otimes 12}\right)$.

- For every closed point $v$ of $\overline{\mathcal{C}}, \min \left(3 \operatorname{ord}_{v}\left(g_{2}\right), 2 \operatorname{ord}_{v}\left(g_{3}\right)\right)<12$.

Then $Y(n, \overline{\mathcal{C}})$ is an open subset of the set of closed points of $\boldsymbol{V}\left(\left(\operatorname{pr}_{2 *}\left(\mathcal{P}_{n}^{\otimes 4} \oplus \mathcal{P}_{n}^{\otimes 6}\right)\right)^{\vee}\right)$. Adding generic points, we obtain an open subscheme of $\boldsymbol{V}\left(\left(\operatorname{pr}_{2 *}\left(\mathcal{P}_{n}^{\otimes 4} \oplus \mathcal{P}_{n}^{\otimes 6}\right)\right)^{\vee}\right)$, which we denote by the same symbol $Y(n, \overline{\mathcal{C}})$ by abuse of notation. Below we only consider $Y(n, \overline{\mathcal{C}})$ as a scheme.

Consider the action of $\mathbb{G}_{m, \operatorname{Pic}_{\overline{\mathcal{C}} / \overline{\mathbb{F}} q}^{n}}$ on $\boldsymbol{V}\left(\left(\operatorname{pr}_{2 *}\left(\mathcal{P}_{n}^{\otimes 4} \oplus \mathcal{P}_{n}^{\otimes 6}\right)\right)^{\vee}\right)$ minus the zero section given by $\lambda$. $\left(\mathscr{L}, g_{2}, g_{3}\right)=\left(\mathscr{L}, \lambda^{4} g_{2}, \lambda^{6} g_{3}\right)$. The quotient of $\boldsymbol{V}\left(\left(\operatorname{pr}_{2 *}\left(\mathcal{P}_{n}^{\otimes 4} \oplus \mathcal{P}_{n}^{\otimes 6}\right)\right)^{\vee}\right)$ minus the zero section by this action of $\mathbb{G}_{m, \operatorname{Pic}_{\overline{\mathcal{C}} / \overline{\mathbb{F}} q}^{n}}$ is the (total space of) weighted projective bundle associated with the vector bundle $\operatorname{pr}_{2 *}\left(\mathcal{P}_{n}^{\otimes 4} \oplus \mathcal{P}_{n}^{\otimes 6}\right)$ over $\operatorname{Pic}_{\overline{\mathcal{C}} / \overline{\mathbb{F}}_{q}}^{n}$ with weight $(4,6)$ [Dolgachev 1982, §1], which is a universal geometric

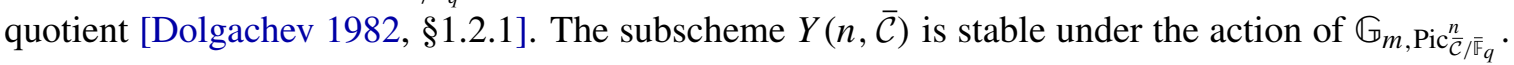

Definition 6.2.2. Define $X(n, \overline{\mathcal{C}})$ to be the quotient of $Y(n, \overline{\mathcal{C}})$ by this action of $\mathbb{G}_{m, \operatorname{Pic}_{\overline{\mathcal{C}} / \overline{\mathbb{F}}_{q}}}$.

Let $k$ be an algebraically closed extension field of $\overline{\mathbb{F}}_{q}$. By [Kas 1977, Theorem 2] (which also works in any characteristic $p>3$, since minimal Weierstrass equations still have only rational double points by [Liu 2002, Chapter 9, Theorem 4.35(a)] and duality $\left.{ }^{7}\right)$, the set of $k$-points of $X(n, \overline{\mathcal{C}})$ corresponds bijectively to the isomorphism classes of minimal elliptic surfaces $\mathcal{S} \rightarrow \mathcal{C}_{k}$ with section and $\chi\left(\mathcal{S}, \mathcal{O}_{\mathcal{S}}\right)=n$. It also corresponds bijectively to the isomorphism classes of elliptic curves over the fraction field of $K \otimes_{\mathbb{F}_{q}} k$ with $\operatorname{deg}(\Delta)=12 n$ by the paragraph after Theorem 5.1.1. For a (not necessarily closed) point $a \in X(n, \overline{\mathcal{C}})$, we define its $\mu$-invariant $\mu_{a}$ to be the $\mu$-invariant of the elliptic curve corresponding to a geometric point (the spectrum of an algebraically closed field) lying over $a$. This defines a function on the underlying set of the scheme $X(n, \overline{\mathcal{C}})$ valued in nonnegative integers.

6.3. Statement and proof. Now the following theorem states that general points of $X(n, \overline{\mathcal{C}})$ correspond to elliptic curves with trivial $\mu$-invariant.

Theorem 6.3.1. Let $p>3$ and $n>\left(g_{\mathcal{C}}-1\right) / 2$ be as above. Then the $\mu$-invariant function $a \mapsto \mu_{a}$ on $X(n, \overline{\mathcal{C}})$ as defined above is upper semicontinuous. The (open) locus where $\mu_{a}=0$ is dense.

Note that if $g_{\mathcal{C}}=0$, then there is no restriction on $n$. We will prove this theorem below. We need some preparations. We first define a universal family of Weierstrass models over $Y(n, \overline{\mathcal{C}})$.

Let $h: Y(n, \overline{\mathcal{C}}) \rightarrow \operatorname{Pic}_{\overline{\mathcal{C}} / \overline{\mathbb{F}}_{q}}^{n}$ be the composite of the inclusion $Y \hookrightarrow \boldsymbol{V}\left(\left(\operatorname{pr}_{2 *}\left(\mathcal{P}_{n}^{\otimes 4} \oplus \mathcal{P}_{n}^{\otimes 6}\right)\right)^{\vee}\right)$ and the natural projection $\boldsymbol{V}\left(\left(\operatorname{pr}_{2 *}\left(\mathcal{P}_{n}^{\otimes 4} \oplus \mathcal{P}_{n}^{\otimes 6}\right)\right)^{\vee}\right) \rightarrow \operatorname{Pic}_{\overline{\mathcal{C}}}^{n} / \overline{\mathbb{F}}_{q}$, which is smooth. Let

$$
h_{\overline{\mathcal{C}}}: \overline{\mathcal{C}} \times Y(n, \overline{\mathcal{C}}) \rightarrow \overline{\mathcal{C}} \times \operatorname{Pic}_{\overline{\mathcal{C}} / \overline{\mathbb{F}}_{q}}^{n}
$$

be the product of the identity on $\overline{\mathcal{C}}$ with $h$. Let $\mathbb{P}\left(h_{\overline{\mathcal{C}}}^{*}\left(\mathcal{P}_{n}^{\otimes 2} \oplus \mathcal{P}_{n}^{\otimes 3} \oplus \mathcal{O}\right)^{\vee}\right)$ be the (total space of) projective bundle associated with the vector bundle $h_{\overline{\mathcal{C}}}^{*}\left(\mathcal{P}_{n}^{\otimes 2} \oplus \mathcal{P}_{n}^{\otimes 3} \oplus \mathcal{O}\right)^{\vee}$ on $\overline{\mathcal{C}} \times Y(n, \overline{\mathcal{C}})$, where $\mathcal{O}$ is the structure sheaf of $\overline{\mathcal{C}} \times \operatorname{Pic}_{\overline{\mathcal{C}} / \overline{\mathbb{F}}_{q}}^{n}$.

\footnotetext{
${ }^{7}$ The exact statement can also be found in Corollary 8.4 of Conrad's notes "Minimal models for elliptic curves" available at http://math.stanford.edu/ conrad/papers/minimalmodel.pdf
} 
Definition 6.3.2. Define $\mathcal{S}^{*}$ to be the closed subscheme of $\mathbb{P}\left(h_{\overline{\mathcal{C}}}^{*}\left(\mathcal{P}_{n}^{\otimes 2} \oplus \mathcal{P}_{n}^{\otimes 3} \oplus \mathcal{O}\right)^{\vee}\right)$ defined by the homogeneous Weierstrass equation $y^{2} z=x^{3}-g_{2} x z^{2}-g_{3} z^{3}$, where $\left(\mathscr{L}, g_{2}, g_{3}\right)$ is any point of $Y(n, \overline{\mathcal{C}})$ (so $g_{2}$ is a global section of $\mathscr{L}^{\otimes 4}$ and $g_{3}$ is a global section of $\mathscr{L}^{\otimes 6}$ ) and $x, y, z$ are coordinates of $\mathcal{P}_{n}^{\otimes 2} \oplus \mathcal{P}_{n}^{\otimes 3} \oplus \mathcal{O}$ (or $\left.\mathscr{L}^{\otimes 2} \oplus \mathscr{L}^{\otimes 3} \oplus \mathcal{O}\right)$.

The equations for the relative affine patches (affine over $\overline{\mathcal{C}} \times Y(n, \overline{\mathcal{C}})$ ) are given by $y^{2}=x^{3}-g_{2} x-g_{3}$ in $\boldsymbol{V}\left(h_{\overline{\mathcal{C}}}^{*}\left(\mathcal{P}_{n}^{\otimes 2} \oplus \mathcal{P}_{n}^{\otimes 3}\right)^{\vee}\right)$ (for the locus $\left.z \neq 0\right)$ and $z=x^{3}-g_{2} x z^{2}-g_{3} z^{3}$ in $\boldsymbol{V}\left(h_{\overline{\mathcal{C}}}^{*}\left(\mathcal{P}_{n}^{\otimes-1} \oplus \mathcal{P}_{n}^{\otimes-3}\right)^{\vee}\right)$ (for the locus $y \neq 0)$. The composite of the inclusion $\mathcal{S}^{*} \hookrightarrow \mathbb{P}\left(h_{\overline{\mathcal{C}}}^{*}\left(\mathcal{P}_{n}^{\otimes 2} \oplus \mathcal{P}_{n}^{\otimes 3} \oplus \mathcal{O}\right)^{\vee}\right)$ and the natural projection $\mathbb{P}\left(h_{\overline{\mathcal{C}}}^{*}\left(\mathcal{P}_{n}^{\otimes 2} \oplus \mathcal{P}_{n}^{\otimes 3} \oplus \mathcal{O}\right)^{\vee}\right) \rightarrow \overline{\mathcal{C}} \times Y(n, \overline{\mathcal{C}})$ defines a morphism $\mathcal{S}^{*} \rightarrow \overline{\mathcal{C}} \times Y(n, \overline{\mathcal{C}})$.

Lemma 6.3.3. - The morphism $\mathcal{S}^{*} \rightarrow \overline{\mathcal{C}} \times Y(n, \overline{\mathcal{C}})$ is projective and flat.

- The fibers of the morphism $\mathcal{S}^{*} \rightarrow Y(n, \overline{\mathcal{C}})$ are normal projective surfaces with only rational double points.

- For any geometric point $b$ of $Y(n, \overline{\mathcal{C}})$, let $\mathcal{S}_{b}^{*}$ be the fiber of $\mathcal{S}^{*} \rightarrow Y(n, \overline{\mathcal{C}})$ over $b$ and $\mathcal{S}_{b}$ the minimal resolution of singularities of $\mathcal{S}_{b}^{*}$. Then for any algebraically closed field $k$ over $\mathbb{F}_{q}$, the map $b \in Y(n, \overline{\mathcal{C}})(k) \mapsto\left[\mathcal{S}_{b} \rightarrow \mathcal{C}_{k}\right]$ gives a bijection between $X(n, \overline{\mathcal{C}})(k)=Y(n, \overline{\mathcal{C}})(k) / k^{\times}$and the set of isomorphism classes of minimal elliptic surfaces over $k$ fibered over $\mathcal{C}_{k}$ with section and $\chi\left(\mathcal{S}_{b}, \mathcal{O}_{\mathcal{S}_{b}}\right)=n$.

Proof. The flatness follows from the above description of the relative affine patches. The rest is [Kas 1977, Theorems 1 and 2] (valid in any characteristic $p>3$ as noted before).

Note that a blowup of $\mathcal{S}^{*}$ does not give a family over $Y(n, \overline{\mathcal{C}})$ with fibers $\mathcal{S}_{b}$ due to exceptional divisors. A simultaneous resolution of singularities for a family of surfaces does not always exist as a scheme [Artin 1974a]. That is why we work with the nonsmooth surfaces $\mathcal{S}_{b}^{*}$.

Lemma 6.3.4. For any geometric point $b$ of $Y(n, \overline{\mathcal{C}})$, the object $\mathrm{Pic}_{\mathcal{S}_{b}^{*} / b}^{0}$ is an abelian variety of dimension independent of $b$.

Proof. If $n=0$, then $\operatorname{deg}(\Delta)=0$ and $g_{\mathcal{C}}=0$. Hence $\mathcal{S}_{b}=\mathcal{S}_{b}^{*} \rightarrow \mathcal{C}_{b}$ is a smooth family of elliptic curves over a projective line, which is necessarily a constant family. Therefore $\mathrm{Pic}_{\mathcal{S}_{b}^{*} / b}^{0}$ is an elliptic curve. Let $n>0$. The morphism $\mathcal{S}_{b}^{*} \rightarrow \mathcal{C}_{b}$ has a section corresponding to $(x: y: z)=(0: 1: 0)$. Hence the morphism $\mathrm{Pic}_{\mathcal{C}_{b} / b} \rightarrow \operatorname{Pic}_{\mathcal{S}_{b}^{*} / b}$ is injective. The group scheme $\mathrm{Pic}_{\mathcal{S}_{b}^{*} / b}^{0}$ is projective by the (geometric) normality of $\mathcal{S}_{b}^{*}$ and [Kleiman 2005, Theorem 5.4]. Its tangent space at zero is $\mathrm{H}^{1}\left(\mathcal{S}_{b}^{*}, \mathcal{O}_{\mathcal{S}_{b}^{*}}\right)$, which is isomorphic to $\mathrm{H}^{1}\left(\mathcal{S}_{b}, \mathcal{O}_{\mathcal{S}_{b}}\right)$ since $\mathcal{S}_{b}^{*}$ has only rational singularities by Lemma 6.3.3. The dimension of this space is $g_{\mathcal{C}}$ by $\operatorname{deg}(\mathcal{L})=n>0$ and the paragraph after Theorem 5.1.1. Therefore $\operatorname{Pic}_{\mathcal{C}_{b} / b}^{0}$ and $\operatorname{Pic}_{\mathcal{S}_{b}^{*} / b}^{0}$ have the same tangent space. Hence $\mathrm{Pic}_{\mathcal{C}_{b} / b}^{0} \stackrel{\sim}{\longrightarrow} \operatorname{Pic}_{\mathcal{S}_{b}^{*} / b}^{0}$, which is an abelian variety of dimension $g_{\mathcal{C}}$.

Lemma 6.3.5. For any geometric point $b$ of $Y(n, \overline{\mathcal{C}})$, we have $\widehat{\mathrm{Br}}_{\mathcal{S}_{b}^{*} / b} \stackrel{\sim}{\rightarrow} \widehat{\mathrm{Br}}_{\mathcal{S}_{b} / b}$, which is a formal Lie group.

Proof. The same argument as the previous lemma shows that $\mathrm{Pic}_{\mathcal{S}_{b} / b}^{0}$ is an abelian variety. Hence the formal Brauer group of $\mathcal{S}_{b}$ is a formal Lie group by [Artin and Mazur 1977, II, Corollary (4.1)] and the 
paragraphs after. By the same argument as [Artin 1974b, (2.1)], the morphism $\widehat{\mathrm{Br}}_{\mathcal{S}_{b}^{*} / b} \rightarrow \widehat{\mathrm{Br}}_{\mathcal{S}_{b} / b}$ is an isomorphism.

Lemma 6.3.6. $\operatorname{Pic}_{\mathcal{S}^{*} / Y(n, \overline{\mathcal{C}})}^{0}$ is represented by a smooth scheme over $Y(n, \overline{\mathcal{C}})$. In particular, the formal completion of $\operatorname{Pic}_{\mathcal{S}^{*} / Y(n, \overline{\mathcal{C}})}$ along the zero section is formally smooth.

Proof. The morphism $\mathcal{S}^{*} \rightarrow Y(n, \overline{\mathcal{C}})$ is projective and flat and its fibers are normal surfaces by Lemma 6.3.3. Hence the sheaf $\operatorname{Pic}_{\mathcal{S}^{*} / Y(n, \overline{\mathcal{C}})}$ is represented by a separated scheme locally of finite type by [Kleiman 2005, Theorem 4.8]. The fibers of $\operatorname{Pic}_{\mathcal{S}^{*} / Y(n, \overline{\mathcal{C}})}^{0}$ are abelian varieties of the same dimension by Lemma 6.3.4. Therefore [Kleiman 2005, Proposition 5.20] implies that $\operatorname{Pic}_{\mathcal{S}^{*} / Y(n, \overline{\mathcal{C}})}^{0}$ is smooth over $Y(n, \overline{\mathcal{C}})$.

Lemma 6.3.7. The formal Brauer group $\widehat{\operatorname{Br}}_{\mathcal{S}^{*} / Y(n, \overline{\mathcal{C}})}$ of $\mathcal{S}^{*} \rightarrow Y(n, \overline{\mathcal{C}})$ exists as a formally smooth formal group scheme over $Y(n, \overline{\mathcal{C}})$. Any point of $Y(n, \mathcal{C})$ has an affine open neighborhood $\mathcal{U}=$ Spec $R$ where $\widehat{\mathrm{Br}}_{\mathcal{S}^{*} / Y(n, \overline{\mathcal{C}})}$ is given by a formal group law over $R$.

Proof. The morphism $\mathcal{S}^{*} \rightarrow Y(n, \overline{\mathcal{C}})$ is cohomologically flat in dimension zero by [EGA III 2 1963, (7.8.6)] since it is flat with geometrically reduced fibers by Lemma 6.3.3. The same lemma says that these fibers are normal projective surfaces. Therefore, with Lemma 6.3.6, we may apply [Artin and Mazur 1977, Corollary (4.1)] and the paragraphs after to see that $\widehat{\mathrm{Br}}_{\mathcal{S}^{*} / Y(n, \overline{\mathcal{C}})}$ exists as a formally smooth formal group scheme over $Y(n, \overline{\mathcal{C}})$. By [Artin and Mazur 1977, II, (2.4) and (3.2)], the Lie algebra of $\widehat{\mathrm{Br}}_{\mathcal{S}^{*} / Y(n, \overline{\mathcal{C}})}$ is the second direct image of the structure sheaf of $\mathcal{S}^{*}$ by the morphism $\mathcal{S}^{*} \rightarrow Y(n, \overline{\mathcal{C}})$. For any point $b \in Y(n, \overline{\mathcal{C}})$, the dimension of $\mathrm{H}^{2}\left(\mathcal{S}_{b}^{*}, \mathcal{O}_{\mathcal{S}_{b}^{*}}\right) \cong \mathrm{H}^{2}\left(\mathcal{S}_{b}, \mathcal{O}_{\mathcal{S}_{b}}\right)$ depends only on $n$ and $g_{\mathcal{C}}$ but not on $b$ by the paragraph after Theorem 5.1.1. Therefore by Grauert's theorem [Hartshorne 1977, III, Corollary 12.9], $\operatorname{Lie}\left(\widehat{\mathrm{Br}}_{\mathcal{S}^{*} / Y(n, \overline{\mathcal{C}})}\right)$ is a vector bundle on $Y(n, \mathcal{C})$. On each open affine $\mathcal{U}=\operatorname{Spec} R$ of $Y(n, \mathcal{C})$ where $\operatorname{Lie}\left(\widehat{\operatorname{Br}}_{\mathcal{S}^{*} / Y(n, \overline{\mathcal{C}})}\right)$ is free, the formal group $\widehat{\mathrm{Br}}_{\mathcal{S}^{*} / Y(n, \overline{\mathcal{C}})}$ is given by a formal group law over $R$ by [Zink 1984, II, Corollary 2.32].

By pullback, the function $a \mapsto \mu_{a}$ on $X(n, \overline{\mathcal{C}})$ defines a function $b \mapsto \mu_{b}$ on $Y(n, \overline{\mathcal{C}})$. The value $\mu_{b}$ is equal to the dimension of the unipotent part of the geometric fiber of $\widehat{\operatorname{Br}}_{\mathcal{S}^{*} / Y(n, \overline{\mathcal{C}})}$ at $b$ by Remark 3.1.4, Proposition 3.1.5 and Lemma 6.3.5.

Lemma 6.3.8. The function $b \mapsto \mu_{b}$ on $Y(n, \overline{\mathcal{C}})$ is upper semicontinuous.

Proof. Set $\mathcal{Y}=Y(n, \overline{\mathcal{C}}), \mathcal{G}=\widehat{\operatorname{Br}}_{\mathcal{S}^{*} / Y(n, \overline{\mathcal{C}})}$ and $d=\operatorname{dim}(\mathcal{G})$. For any nonnegative integer $m$, let $\mathcal{G}\left[F^{m}\right]$ be the kernel of the $p^{m}$-th power relative Frobenius morphism $\mathcal{G} \rightarrow \mathcal{G}^{\left(p^{m}\right)}$ over $\mathcal{Y}$. Then $\mathcal{G}\left[F^{m}\right]$ is finite flat over $\mathcal{Y}$ by Lemma 6.3.7. Hence the intersection $\mathcal{G}\left[F^{m}\right] \cap \mathcal{G}\left[p^{d}\right]$ of $\mathcal{G}\left[F^{m}\right]$ and the $p^{d}$-torsion part $\mathcal{G}\left[p^{d}\right]$ is finite over $\mathcal{Y}$.

For any $b \in \mathcal{Y}$, let $\mathcal{G}_{b \text {,uni }}$ be the unipotent part of the fiber $\mathcal{G}_{b}$ and $\mathcal{G}_{b \text {,div }}$ the $p$-divisible quotient of $\mathcal{G}_{b}$ [Zink 1984, V, $\S 8$, Theorem 5.36]. Let $h_{b}$ be the height of $\mathcal{G}_{b, \text { div }}$. Let $h_{b, m}$ be the log base $p$ of the degree of the finite group scheme $\mathcal{G}_{b}\left[F^{m}\right] \cap \mathcal{G}_{b}\left[p^{d}\right]$ over $b$. We first show that

$$
m \mu_{b} \leq h_{b, m} \leq m \mu_{b}+d h_{b} .
$$

The exact sequence $0 \rightarrow \mathcal{G}_{b \text {, uni }} \rightarrow \mathcal{G}_{b} \rightarrow \mathcal{G}_{b \text {,div }} \rightarrow 0$ induces an exact sequence

$$
0 \rightarrow \mathcal{G}_{b, \text { uni }}\left[F^{m}\right] \cap \mathcal{G}_{b, \text { uni }}\left[p^{d}\right] \rightarrow \mathcal{G}_{b}\left[F^{m}\right] \cap \mathcal{G}_{b}\left[p^{d}\right] \rightarrow \mathcal{G}_{b, \operatorname{div}}\left[F^{m}\right] \cap \mathcal{G}_{b, \operatorname{div}}\left[p^{d}\right]
$$


Since $\mathcal{G}_{b \text {, uni }}$ is killed by $p^{d}$, the first term $\mathcal{G}_{b \text {, uni }}\left[F^{m}\right] \cap \mathcal{G}_{b \text {, uni }}\left[p^{d}\right]$ is equal to $\mathcal{G}_{b \text {, uni }}\left[F^{m}\right]$, whose log base $p$ of the degree is $m \mu_{b}$. The third term $\mathcal{G}_{b, \mathrm{div}}\left[F^{m}\right] \cap \mathcal{G}_{b, \mathrm{div}}\left[p^{d}\right]$ is contained in $\mathcal{G}_{b, \mathrm{div}}\left[p^{d}\right]$, whose log base $p$ of the degree is $d h_{b}$. These show the desired inequalities.

Set $m=m_{b}=d h_{b}+1$. Let $\mathcal{U}_{b}$ be the set of points $c \in \mathcal{Y}$ such that $h_{c, m} \leq h_{b, m}$. By the finiteness of $\mathcal{G}\left[F^{m}\right] \cap \mathcal{G}\left[p^{d}\right]$ over $\mathcal{Y}$ and Nakayama's lemma, we know that $\mathcal{U}_{b}$ is an open subset of $\mathcal{Y}$ and hence an open neighborhood of $b$. For any $c \in \mathcal{U}_{b}$, we have

$$
m \mu_{c} \leq h_{c, m} \leq h_{b, m} \leq m \mu_{b}+d h_{b}=m\left(\mu_{b}+1\right)-1,
$$

so $\mu_{c} \leq \mu_{b}$. This shows that $b \mapsto \mu_{b}$ is upper semicontinuous.

Now we prove Theorem 6.3.1.

Proof of Theorem 6.3.1. By Lemma 6.3.8, the locus of $Y(n, \overline{\mathcal{C}})$ where $\mu_{b}=0$ (or equivalently, $\mu_{b}<1$ ) is open. We show that this locus is dense. The scheme $\operatorname{Pic}_{\overline{\mathcal{C}} / \overline{\mathbb{F}}_{q}}^{n}$ is irreducible since for any choice of a closed point $P$ of $\overline{\mathcal{C}}$, the map $D \mapsto D+P$ of divisors defines an isomorphism $\operatorname{Pic}_{\overline{\mathcal{C}} / \overline{\mathbb{F}}_{q}}^{0} \stackrel{\sim}{\rightarrow} \operatorname{Pic}_{\overline{\mathcal{C}} / \overline{\mathbb{F}}_{q}}^{n}$ from the Jacobian variety $\operatorname{Pic}_{\overline{\mathcal{C}} / \overline{\mathbb{F}}_{q}}^{0}$ of $\overline{\mathcal{C}}$. Hence the vector bundle $V\left(\left(\operatorname{pr}_{2 *}\left(\mathcal{P}_{n}^{\otimes 4} \oplus \mathcal{P}_{n}^{\otimes 6}\right)\right)^{\vee}\right)$ over it is also irreducible. Hence so is its nonempty open subscheme $Y(n, \overline{\mathcal{C}})$. The locus where $\mu=0$ on $X(n, \overline{\mathcal{C}})$ or $Y(n, \overline{\mathcal{C}})$ is nonempty by Proposition 6.1.1. A nonempty open subset of an irreducible topological space is dense. Therefore the locus of $Y(n, \overline{\mathcal{C}})$ with $\mu_{b}=0$ is dense.

As we saw before, $X(n, \overline{\mathcal{C}})$ is a universal geometric quotient of $Y(n, \overline{\mathcal{C}})$. In particular, $X(n, \overline{\mathcal{C}})$ has a quotient topology of $Y(n, \overline{\mathcal{C}})$. Hence the function $a \mapsto \mu_{a}$ on $X(n, \overline{\mathcal{C}})$ is also upper semicontinuous, and the locus of $X(n, \overline{\mathcal{C}})$ with $\mu_{a}=0$ is dense.

If the condition $n>\left(g_{\mathcal{C}}-1\right) / 2$ is not satisfied, the scheme structure on the set $X(n, \overline{\mathcal{C}})$ is much more complicated; see [Seiler 1987]. In particular, it is not necessarily irreducible. It is not clear if the theorem extends to this case.

Remark 6.3.9. The variety $X(n, \overline{\mathcal{C}})$ over $\overline{\mathbb{F}}_{q}$ can be naturally defined over a finite extension of $\mathbb{F}_{q}$ where $\mathcal{C}$ has a rational point (so that a Poincaré sheaf is well-defined). Assume that $\mathcal{C}$ has a rational point and denote the resulting variety over $\mathbb{F}_{q}$ by $X(n, \mathcal{C})$. The residue fields at closed points of $X(n, \mathcal{C})$ are finite. Therefore Theorem 6.3.1 says that there are "many" elliptic curves over finite constant extensions of $K$ with given $\operatorname{deg}(\Delta)$ and $\mu=0$, where this $\mu$ means the "usual" $\mu$-invariant as defined in Definition 2.0.1. More specifically, the $\mu=0$ locus $X(n, \mathcal{C})_{\mu=0}$ is a dense open subvariety of $X(n, \mathcal{C})$. Hence the set of closed point of $X(n, \mathcal{C})_{\mu=0}$ with the induced Zariski topology is dense open in the set of closed points of $X(n, \mathcal{C})$.

\section{Computations}

In this section, we give a certain number of examples where we are able to calculate the $\mu$-invariant.

7.1. Legendre curves. Assume that $\operatorname{char}(K) \neq 2$ and $A$ is defined by the Legendre form,

$$
y^{2}=x(x-1)(x-f) .
$$


For each nonzero $g \in K$, let $(g)_{0}$ and $(g)_{\infty}$ denote the divisors of zeros and poles of $g$. Write

$$
N=\sum_{v} n_{v} \cdot v, \quad \text { and } \quad \Delta=\sum_{v} \delta_{v} \cdot v .
$$

For a place $v$, let $\mathbb{F}_{v}$ denote the residue field and choose a local parameter $\pi_{v}$. Suppose $v$ does not divide $(f)_{\infty}$ and let $\bar{f} \in \mathbb{F}_{v}$ denote the residue class of $f$ at $v$. Then the equation

$$
y^{2}=x(x-1)(x-\bar{f})
$$

defines a curve over $\mathbb{F}_{v}$ having at worst a node. Hence it actually defines $\bar{A}$, the reduction of $A$ at $v$, so (23) is a minimal equation. Furthermore, if $v$ does not divide $(f)_{0}+(f-1)_{0}$, then $\bar{A}$ is an elliptic curve, in this case, we have

$$
n_{v}=0, \quad \text { and } \quad \delta_{v}=0 .
$$

In the case where $v$ divides $(f)_{0}$, Equation (24) reads $y^{2}=x^{2}(x-1)$. Put $y=\xi \cdot x$, then for $(x, y) \neq(0,0)$, the singularity of $\bar{A}$, we have $x=\xi^{2}+1, y=\xi\left(\xi^{2}+1\right)$. Thus, $A$ has split multiplicative reduction if -1 is a square in $\mathbb{F}_{v}$; nonsplit multiplicative reduction, if -1 is not a square. Let $m_{v}$ be the coefficient of $v$ in $(f)_{0}$ so that $f=\pi^{m_{v}} u$, for some $u \in \mathcal{O}_{v}$. Then the discriminant of (23) equals

$$
\left(\pi_{v}^{m_{v}} u\left(\pi_{v}^{m_{v}} u-1\right)\right)^{2}=\pi^{2 m_{v}} u^{\prime}, \quad u^{\prime} \in \mathcal{O}_{v}^{*} .
$$

Thus, in this case, we have

$$
n_{v}=1, \quad \text { and } \quad \delta_{v}=2 m_{v} .
$$

If $v$ divides $(f-1)_{0}$, Equation (24) becomes $y^{2}=x(x-1)^{2}$. By putting $y=\xi \cdot(x-1)$ and by an argument similar to the above, we deduce that $A$ has split multiplicative reduction at $v$ and if $m_{v}$ is the coefficient of $v$ in $(f-1)_{0}$, then also

$$
n_{v}=1, \quad \text { and } \quad \delta_{v}=2 m_{v} .
$$

Call a divisor $C=\sum c_{v} \cdot v$ even, if all $c_{v}$ are even integers.

Lemma 7.1.1. The elliptic curve $A$ has semistable reduction at all places, if and only if $(f)_{\infty}$ is even.

Proof. Suppose $m_{v}>0$ is the coefficient of a place $v$ in $(f)_{\infty}$ so that $f=\pi^{-m_{v}} u$, for some $u \in \mathcal{O}_{v}^{*}$. If $m_{v}=2 e$ is even, then by replacing $(x, y)$ with $\left(x \pi_{v}^{-2 e}, y \pi_{v}^{-3 e}\right)$, Equation (23) becomes

$$
y^{2}=x\left(x-\pi_{v}^{2 e}\right)(x-u) .
$$

This turns out to be a minimal equation with multiplicative reduction. In this case,

$$
n_{v}=1, \quad \text { and } \quad \delta_{v}=2 m_{v}
$$

On the other hand, if $m_{v}=2 e-1$ is odd, then by replacing $(x, y)$ with $\left(x \pi_{v}^{-2 e}, y \pi_{v}^{-3 e}\right)$, Equation (23) becomes

$$
y^{2}=x\left(x-\pi_{v}^{2 e}\right)\left(x-u \pi_{v}\right)
$$


which is also a minimal equation (see [Silverman 1986, §VI.1]) with additive reduction.

If $(f)_{\infty}$ is even, then the equalities (25)-(28) together imply

$$
\operatorname{deg} \Delta=6 \operatorname{deg} f
$$

7.2. A simple example. Let $K=\mathbb{F}_{q}(t)$. Here we demonstrate the case of lower degree $f{ }^{8}$ Take $f(t)=g(t) / t^{2}, g(t) \in \mathbb{F}_{q}(t), t \nmid g(t), \operatorname{deg} g(t) \leq 2$, so $\operatorname{deg} f=2$ and hence

$$
\operatorname{deg} \Delta=12 .
$$

Therefore $\mu_{A / K}=0$ by Theorem 5.1.1. The associated surface is rational (hence has trivial Brauer group over $\overline{\mathbb{F}}_{q}$ ) by the paragraph after Theorem 5.1.1.

7.3. Computation using Magma. In this subsection, we examine the $\mu$-invariant of an elliptic curve over $K$ other than a rational function field using Magma [Bosma et al. 1997]. We limit ourselves to an elliptic curve over a quadratic extension of a rational function field since Magma cannot treat curves over cubic extensions or higher at the time we wrote the paper.

Let $\mathcal{C} / \mathbb{F}_{7}$ be the supersingular elliptic curve with Weierstrass equation $Y^{2}=X^{3}-5 X$. We denote by $K$ its function field. It is a quadratic extension of the rational function field $\mathbb{F}_{7}(x)$. Let $E / \mathbb{F}_{7}(x)$ be the elliptic curve given by the Legendre form $Y^{2}=X(X-1)\left(X-1 / x^{2}\right)$. Then, the $L$-function of $E / K$ can be computed as the product $L_{E}(s) \cdot L_{\mathrm{tw}(E)}(s)$, where $\operatorname{tw}(E) / \mathbb{F}_{7}(x)$ is the quadratic twist of $E$ by the element $x^{3}+5 x$ of $\mathbb{F}_{7}(x)$. The integers $\operatorname{deg}(\Delta)$ and $\theta$ of $E / K$ are computed as follows: First to compute $\theta$ we just need to know $P(T)$ such that $P\left(7^{-s}\right)=L_{E}(s)$. This information is obtained by the following Magma command:

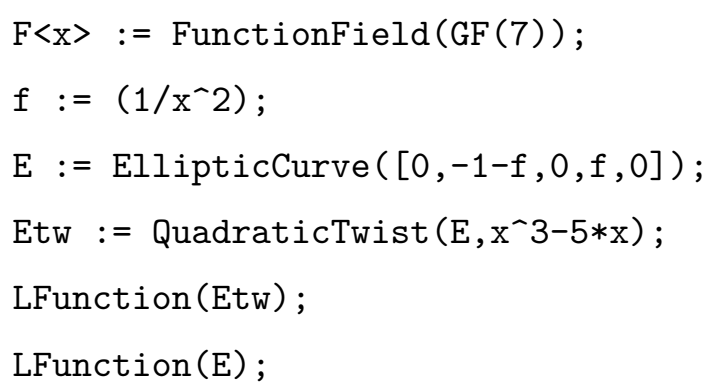

We obtain

$-117649 * \mathrm{~T}^{\wedge} 6+1715 * \mathrm{~T}^{\wedge} 4-35 * \mathrm{~T}^{\wedge} 2+1$

1

By hand computation, we know that $7^{2} P\left(7^{-1} T\right)$ is 7-primitive, so $\theta=2$.

Next, to implement the curve $E / K$, we use the Magma command:

$$
\mathrm{S}\langle z>\text { := PolynomialRing }(\mathrm{F}) \text {; }
$$

\footnotetext{
${ }^{8}$ Here the degree means that of $(f)_{0}$, or $(f)_{\infty}$.
} 


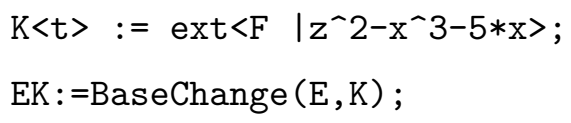

The integer $\operatorname{deg}\left(\Delta_{E / K}\right)$ is obtained by the following command:

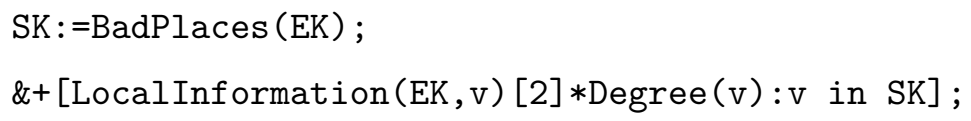

We obtain $\operatorname{deg}\left(\Delta_{E / K}\right)=24$ and conclude that the $\mu$-invariant of $E / K$ is zero by Theorem 5.1.1. The associated surface has Kodaira dimension 1 by the paragraph after Theorem 5.1.1.

7.4. An example of nonsemistable reduction. We consider the curve $y^{2}=x(x-1)(x-f(t))$ with $f:=\left(1+t+t^{2}\right) /(1+t) \in \mathbb{F}_{3}[t]$ on Magma. The output is the following:

(a) The bad places are $(t+1)_{0},(1 / t)_{0}$, those are additive places, and $(t+2)_{0},(t)_{0}$, multiplicative places.

(b) The $j$-invariant is

$$
j=\frac{t^{12}+t^{9}+2 t^{6}+2 t^{3}+1}{t^{10}+t^{9}+2 t^{8}+t^{7}+2 t^{6}+t^{5}+t^{4}}=\frac{t^{12}+t^{9}+2 t^{6}+2 t^{3}+1}{(t+1)^{2}(t-1)^{4} t^{4}} .
$$

One can compute by hand that

$$
\Delta=8(t+1)_{0}+8(1 / t)_{0}+4(t)_{0}+4(t+2)_{0},
$$

so $\operatorname{deg} \Delta=24$. Equation (29) is not satisfied here. Note that $j=c_{4}^{3} / \Delta$, but in this case, $\Delta$ is not the exact "denominator" of $j$.

(c) $P(T)=-9 T^{2}+1$, so $\theta=0$.

Therefore $\mu=1$. The associated surface is a $\mathrm{K} 3$ surface, and the result $\mu=1$ shows that it is supersingular.

7.5. A supersingular isotrivial curve. We give an example of an isotrivial nonconstant elliptic curve with everywhere good supersingular reduction and $\mu=0$ over a function field with noninvertible Hasse-Witt matrix. As any finite extension of such a function field has noninvertible Hasse-Witt matrix, this gives a counterexample to the "only if" direction of [Ochiai and Trihan 2009, Theorem 1.8(2)].

Assume $p \neq 2$. Let $\mathcal{C}^{\prime} \rightarrow \mathcal{C}$ be a degree 2 isogeny between supersingular elliptic curves over $\mathbb{F}_{q}$. Let $K^{\prime} / K$ be the corresponding extension of function fields. Let $A_{0} / K$ be a constant supersingular elliptic curve. Let $A / K$ be the quadratic twist of $A_{0}$ by $K^{\prime} / K$, which has good reduction everywhere since $K^{\prime} / K$ is unramified everywhere. The elliptic surfaces over $\mathbb{F}_{q}$ corresponding to $A_{0} / K$ and $A_{0} / K^{\prime}$ are both supersingular abelian surfaces. Therefore $\mu_{A_{0} / K}=\mu_{A_{0} / K^{\prime}}=1$ by Proposition 3.4.4. As $p \nmid\left[K^{\prime}: K\right]=2$, the $\Lambda$-module $\operatorname{Sel}_{p^{\infty}}\left(A_{0} / K_{\infty}^{\prime(p)}\right)$ is isomorphic to $\operatorname{Sel}_{p^{\infty}}\left(A / K_{\infty}^{(p)}\right) \oplus \operatorname{Sel}_{p^{\infty}}\left(A_{0} / K_{\infty}^{(p)}\right)$. Therefore $\mu_{A / K}=\mu_{A_{0} / K^{\prime}}-\mu_{A_{0} / K}=0$. This $A / K$ thus gives a desired example.

One checks that the elliptic surface corresponding to $A / K$ is a hyperelliptic surface. As such, we have $\mu_{A / K}=0$ also by Proposition 3.4.4. More generally, for any hyperelliptic surface $\mathcal{S}$ obtained as a quotient 
of the product of two supersingular elliptic curves [Bombieri and Mumford 1977, Theorem 4], the generic fiber $A / K$ of its Albanese morphism $\mathcal{S} \rightarrow \mathcal{C}$ gives a desired example. This includes the case of $p=2$.

What is true about the "only if" direction of [Ochiai and Trihan 2009, Theorem 1.8(2)] and what the proof of this statement in [Ochiai and Trihan 2009] actually says is that for a constant nonzero supersingular abelian variety $A / K$, if $\mu_{A / K}=0$, then the Hasse-Witt matrix of $K$ is invertible.

7.6. Large rank curves of Ulmer and Shioda. We compute the $\mu$-invariants of the elliptic curves with large ranks considered by Ulmer [2002, Theorem 1.5] and Shioda [1986, Remark 10]. This is an application of the method of proof of Proposition 5.1.2.

Ulmer's elliptic curve is defined by the equation $y^{2}+x y=x^{3}-t^{d}$ over $K=\mathbb{F}_{p}(t)$, where $p$ is an arbitrary prime, $d=p^{n}+1$ and $n$ a positive integer. As explained in [Ulmer 2002, §10.2], its geometric Mordell-Weil rank $\bar{r}$ attains the geometric bound, i.e., $\bar{r}=a\left(=\operatorname{deg}\left(P_{1}(t)\right)\right.$; see (4)). This implies $\theta=0$ by the proof of Proposition 5.1.2. Therefore $\mu=\operatorname{deg}(\Delta) / 12-1$ by Theorem 5.1.1. The calculations in [Ulmer 2002, §2] show that $\operatorname{deg}(\Delta) / 12=\lceil d / 6\rceil$ (the least integer $\geq d / 6$ ). Hence $\mu=\lceil d / 6\rceil-1$.

On the other hand, Shioda's elliptic curve is defined by the equation $y^{2}=x^{3}+x+t^{d}$ over $K=\mathbb{F}_{p}(t)$, where $p \equiv-1 \bmod 4, d=\left(p^{v}+1\right) / 2$ and $v$ is a positive odd integer. Let $\mathcal{S} / \mathbb{F}_{p}$ be the elliptic surface corresponding to this curve. Then its geometric Lefschetz number $\lambda\left(\mathcal{S}_{\overline{\mathbb{F}}_{p}}\right)=\operatorname{deg} P_{\mathcal{S}, 2}-\rho_{\mathcal{S}_{\bar{F}_{p}}}$ is zero as shown in [Shioda 1986, Remark 10]. Therefore $\theta=0$ again by the proof of Proposition 5.1.2. Therefore $\mu=\operatorname{deg}(\Delta) / 12-1$ by Theorem 5.1.1. A calculation similar to [Ulmer 2002, §2] shows that $\operatorname{deg}(\Delta) / 12=\lceil d / 6\rceil$. Hence $\mu=\lceil d / 6\rceil-1$.

7.7. Other examples in the literature. Numerous other computations can be found in [Ulmer 2019] (for example [Ulmer 2019, Remark 4.5, Theorem 5.1, Corollary 6.5 and its applications in Sections 10-12]).

\section{Appendix: Integrality of sum of slopes less than one}

In this appendix, we show that the number $\theta$ defined in Definition 2.2.2 is an integer. We follow [Trihan and Yasuda 2014].

Let $A / K$ be an abelian variety. Let $\mathcal{U}$ be an affine open subscheme of $\mathcal{C}$ where $A$ has good reduction. Recall from [Trihan and Yasuda 2014, Equation (6)] the following long exact sequence

$$
\cdots \rightarrow \mathrm{H}_{\mathrm{rig}, c}^{i}\left(\mathcal{U}, D^{\dagger}(A)\right) \rightarrow \mathrm{H}_{\mathrm{rig}}^{i}\left(\mathcal{U}, D^{\dagger}(A)\right) \rightarrow \mathrm{H}^{i}\left(\operatorname{DR}_{A_{\mathcal{U}}^{\text {loc }}}\left(D^{\dagger}(A)\right)\right) \rightarrow \cdots
$$

of $F$-isocrystals over $\mathbb{F}_{q}$, where $D^{\dagger}(A)$ is the $F$-overconvergent isocrystal associated with $A, \mathrm{H}_{\text {rig }}^{i}$ rigid cohomology and $\mathrm{H}_{\mathrm{rig}, c}^{i}$ its version with compact support (see [Trihan and Yasuda 2014, §4.2] for the definition of the third term). We write the ( $p$-th power) Frobenius map for any of these $F$-isocrystals by $\varphi_{p}$ and set $\varphi_{q}=\varphi_{p}^{e}$. As in the paragraph after [Trihan and Yasuda 2014, Remark 4.2.1], let $\mathcal{H}_{\mathbb{Q}_{p}}^{1}$ be the image of the map $\mathrm{H}_{\mathrm{rig}, c}^{1}\left(\mathcal{U}, D^{\dagger}(A)\right) \rightarrow \mathrm{H}_{\mathrm{rig}}^{1}\left(\mathcal{U}, D^{\dagger}(A)\right)$. All the determinants below are taken over $W\left(\mathbb{F}_{q}\right)[1 / p]$.

Proposition A.1. $P_{1}(t)=\operatorname{det}\left(1-\varphi_{q} t \mid \mathcal{H}_{\mathbb{Q}_{p}}^{1}(-1)\right)$, where $(-1)$ denotes a Tate twist. 
Proof. We fix an embedding $\iota: W\left(\mathbb{F}_{q}\right)[1 / p] \hookrightarrow \mathbb{C}$. Since $D^{\dagger}(A)$ is $\iota$-pure of weight -1 by [Trihan and Yasuda 2014, Lemma 4.2.3], we know that $\mathrm{H}_{\text {rig }}^{0}\left(\mathcal{U}, D^{\dagger}(A)\right)$ is $\iota$-pure of weight -1 by the third paragraph of [Kedlaya 2006, §6.1]. Dualizing, $\mathrm{H}_{\text {rig, },}^{2}\left(\mathcal{U}, D^{\dagger}(A)\right)$ is $\iota$-pure of weight 1. By the proof of [Trihan and Yasuda 2014, Lemma 4.2.4], $\mathrm{H}^{0}\left(\operatorname{DR}_{A_{\mathcal{U}}^{\text {loc }}}\left(D^{\dagger}(A)\right)\right)$ is $\iota$-mixed of weights $\leq-1$. Also, $\mathrm{H}_{\text {rig, },}^{1}\left(\mathcal{U}, D^{\dagger}(A)\right)$ is $\iota$-mixed of weights $\leq 0$ by [Kedlaya 2006, 6.6.2(a)]. Similarly, $\mathrm{H}_{\text {rig }}^{1}\left(\mathcal{U}, D^{\dagger}(A)\right)$ is $\iota$-mixed of weights $\geq 0$.

Hence in the exact sequence

$$
\mathrm{H}^{0}\left(\operatorname{DR}_{A_{\mathcal{U}}^{\text {loc }}}\left(D^{\dagger}(A)\right)\right) \rightarrow \mathrm{H}_{\text {rig }, c}^{1}\left(\mathcal{U}, D^{\dagger}(A)\right) \rightarrow \mathrm{H}_{\text {rig }}^{1}\left(\mathcal{U}, D^{\dagger}(A)\right)
$$

the terms are $\iota$-mixed of weights $\leq-1, \leq 0$ and $\geq 0$, respectively. Therefore the image of the second map, which is $\mathcal{H}_{\mathbb{Q}_{p}}^{1}$, is the maximal quotient of $\mathrm{H}_{\text {rig,c }}^{1}\left(\mathcal{U}, D^{\dagger}(A)\right)$ l-pure of weight 0 .

Let $\mathcal{Z}=\mathcal{C} \backslash \mathcal{U}$. We have $\mathrm{H}_{\text {rig, } c}^{0}\left(\mathcal{U}, D^{\dagger}(A)\right)=0$ since $\mathrm{H}_{\text {rig, },}^{i+1}\left(\mathcal{U}, D^{\dagger}(A)\right)$ is the $i$-th cohomology of a complex concentrated in degrees 0 and 1 by [Trihan and Yasuda 2014, §4.2]. Therefore the $L$-function $L_{A, \mathcal{Z}}(s)$ can be written as

$$
\frac{\operatorname{det}\left(1-\varphi_{q} t \mid \mathrm{H}_{\text {rig }, c}^{1}\left(\mathcal{U}, D^{\dagger}(A)\right)(-1)\right)}{\operatorname{det}\left(1-\varphi_{q} t \mid \mathrm{H}_{\mathrm{rig}, c}^{2}\left(\mathcal{U}, D^{\dagger}(A)\right)(-1)\right)}
$$

by [Kato and Trihan 2003, 4.3], where $t=q^{-s}$. As seen above, the reciprocal roots of the numerator are Weil $q$-numbers of weights $\leq 2$ (via the embedding $\iota$ ) and the reciprocal roots of the denominator are Weil $q$-numbers of weight 3 . The same function $L_{A, \mathcal{Z}}(s)$ can also be written as

$$
\frac{P_{1}(t)}{P_{0}(t) P_{2}(t)} \cdot \prod_{v \in \mathcal{Z}} P_{v}\left(t_{v}\right),
$$

where $t_{v}=t^{\operatorname{deg}(v)}$ and $P_{v}\left(t_{v}\right)$ is the Euler factor at $v$. The reciprocal roots of $P_{i}(t)$ are Weil $q$-numbers of weight $i+1$ and the reciprocal roots of $P_{v}\left(t_{v}\right)$ are Weil $q_{v}$-numbers of weights 0 and 1. Comparing the weights of the zeros and poles of these two expressions of $L_{A, \mathcal{Z}}(s)$, we know that the maximal quotient of $\operatorname{det}\left(1-\varphi_{q} t \mid \mathrm{H}_{\text {rig, } c}^{1}\left(\mathcal{U}, D^{\dagger}(A)\right)(-1)\right)$ with reciprocal roots of weight 2 is $P_{1}(t)$. Hence $\operatorname{det}\left(1-\varphi_{q} t \mid \mathcal{H}_{\mathbb{Q}_{p}}^{1}(-1)\right)$ is $P_{1}(t)$.

Proposition A.2. Let $\left(M, \varphi_{p}\right)$ be an $F$-isocrystal over $\mathbb{F}_{q}$. Then the vertices of the Newton polygon of $\operatorname{det}\left(1-\varphi_{q} t \mid M\right)$ with respect to the q-valuation have integer coordinates, where $\varphi_{q}=\varphi_{p}^{e}$.

Proof. First assume that $M$ is isoclinic. Let $n$ be the dimension of $M$ over $W\left(\mathbb{F}_{q}\right)[1 / p]$. Let $\lambda=s / r$ be the slope of $M$, where $s$ and $r$ are coprime integers with $r>0$. Then $M$ admits a $W\left(\mathbb{F}_{q}\right)$-lattice $M_{0}$ such that $\varphi_{p}^{r}\left(M_{0}\right)=p^{s} M_{0}$ by the Dieudonné-Manin classification over $\overline{\mathbb{F}}_{q}$ and Galois descent. Hence $\varphi_{q}^{r}\left(M_{0}\right)=q^{s} M_{0}$. Therefore the $q$-valuations of the reciprocal roots of $\operatorname{det}\left(1-\varphi_{q} t \mid M\right)$ are $\lambda$. Hence the vertices of the Newton polygon of $\operatorname{det}\left(1-\varphi_{q} t \mid M\right)$ with respect to the $q$-valuation are $(0,0)$ and $(n, n \lambda)$. The number $n \lambda$ is an integer by the Dieudonné-Manin classification over $\overline{\mathbb{F}}_{q}$.

The general case follows from this calculation and the slope decomposition. 
Proof. By Proposition A.1, $P_{1}(t)$ is the characteristic polynomial of the $q$-th power Frobenius action on an $F$-isocrystal over $\mathbb{F}_{q}$. Hence the vertices of the Newton polygon of $P_{1}(t)$ with respect to the $q$-valuation have integer coordinates by Proposition A.2. In particular, $\sum_{\lambda_{j}<1} \lambda_{j} \in \mathbb{Z}$. This implies that $\theta \in \mathbb{Z}$ by Proposition 2.2.3.

Remark A.4. (1) To see how nontrivial this statement $\theta \in \mathbb{Z}$ is, consider the case $q=4$ and the polynomial $P^{\prime}(t)=1-2 t+16 t^{2}$ instead of $P_{1}(t)$. The reciprocal roots of $P^{\prime}(t)$ are Weil $q$-numbers of weight 2 , just as $P_{1}(t)$. The polynomial $P^{\prime}(t)$ also satisfies the expected functional equation $P^{\prime}(t)=16 t^{2} P^{\prime}(1 / 16 t)$. The $q$-valuations $\left\{\lambda_{j}^{\prime}\right\}$ of the reciprocal roots of $P^{\prime}(t)$ are $\frac{1}{2}$ and $\frac{3}{2}$, which are numbers between 0 and 2 invariant under $\lambda_{j}^{\prime} \leftrightarrow 2-\lambda_{j}^{\prime}$. Therefore $P^{\prime}(t)$ shares most of the properties of $P_{1}(t)$. Yet $\sum_{\lambda_{j}^{\prime}<1} \lambda_{j}^{\prime}$ is $\frac{1}{2}$, which is not an integer. It is crucial that $P_{1}(t)$ comes from an $F$-isocrystal over $\mathbb{F}_{q}$.

(2) In the proof of [Ulmer 2019, 4.1], it is stated that since the break points of a Newton polygon have integer coordinates, $\sum_{\lambda_{i}<1}\left(\lambda_{i}-1\right)$ is an integer. A possible interpretation of this line is the combination of the above three propositions. This integrality is used to prove that $\operatorname{dim} \amalg(A)$ in the notation of [Ulmer 2019, 4.1] is an integer.

(3) In the case $A$ is a Jacobian, the integrality of $\theta\left(=\theta_{A}\right)$ also follows from the Crew-Milne formula (14) for surfaces (so $\theta_{\mathcal{S}} \in \mathbb{Z}$ ) and Proposition 3.3.1.

(4) In [Kahn 2014, Theorem 1(b)], it is stated that $P_{1}(t)$ is the reciprocal of the " $Z$-function" of a certain Chow motive over $\mathbb{F}_{q}$. If we used this statement, then $P_{1}(t)$ would be expressed as the characteristic polynomial of the $q$-th power Frobenius on the crystalline realization of this Chow motive by [Kahn 2014, Proposition 3.4 and Lemma 5.1]. This could be used instead of Proposition A.1 to give another proof of $\theta \in \mathbb{Z}$. Unfortunately, the proof of [Kahn 2014, Theorem 1 b)] (especially [Kahn 2014, Proposition 3.5]) is valid only for the case $A$ is a Jacobian, as pointed out in the paragraphs after [Kahn 2018b, théorème 8.6].

\section{Acknowledgments}

The authors are grateful to Luc Illusie, Bruno Kahn, Masato Kurihara, Kentaro Mitsui, Yukiyoshi Nakkajima, Tadashi Ochiai, Atsushi Shiho, Douglas Ulmer and Christian Wuthrich for helpful discussions. Many thanks also to the referee for the careful reading and the comments, which helped to significantly improve the paper.

\section{References}

[Anglès et al. 2020] B. Anglès, A. Bandini, F. Bars, and I. Longhi, "Iwasawa main conjecture for the Carlitz cyclotomic extension and applications", Math. Ann. 376:1-2 (2020), 475-523. MR Zbl

[Artin 1974a] M. Artin, "Algebraic construction of Brieskorn's resolutions", J. Algebra 29 (1974), 330-348. MR Zbl [Artin 1974b] M. Artin, "Supersingular K3 surfaces", Ann. Sci. École Norm. Sup. (4) 7 (1974), 543-567. MR Zbl

[Artin and Mazur 1977] M. Artin and B. Mazur, "Formal groups arising from algebraic varieties", Ann. Sci. École Norm. Sup.

(4) 10:1 (1977), 87-131. MR Zbl

[Bombieri and Mumford 1977] E. Bombieri and D. Mumford, "Enriques' classification of surfaces in char. $p$, II", pp. 23-42 in Complex analysis and algebraic geometry, edited by W. L. Baily, Jr., Iwanami, Tokyo, 1977. MR Zbl 
[Bosch et al. 1990] S. Bosch, W. Lütkebohmert, and M. Raynaud, Néron models, Ergebnisse der Mathematik (3) 21, Springer, 1990. MR Zbl

[Bosma et al. 1997] W. Bosma, J. Cannon, and C. Playoust, "The Magma algebra system, I: The user language”, J. Symbolic Comput. 24:3-4 (1997), 235-265. MR Zbl

[Coates and Sujatha 2005] J. Coates and R. Sujatha, "Fine Selmer groups of elliptic curves over p-adic Lie extensions", Math. Ann. 331:4 (2005), 809-839. MR Zbl

[Coates and Sujatha 2006] J. Coates and R. Sujatha, Cyclotomic fields and zeta values, Springer, 2006. MR Zbl

[Conrad 2006] B. Conrad, "Chow's $K / k$-image and $K / k$-trace, and the Lang-Néron theorem", Enseign. Math. (2) 52:1-2 (2006), 37-108. MR Zbl

[Demazure and Gabriel 1970] M. Demazure and P. Gabriel, Groupes algébriques, I: Géométrie algébrique, généralités, groupes commutatifs, Masson \& Cie, Paris, 1970. MR Zbl

[Dolgachev 1982] I. Dolgachev, "Weighted projective varieties", pp. 34-71 in Group actions and vector fields (Vancouver, 1981), edited by J. B. Carrell, Lecture Notes in Math. 956, Springer, 1982. MR Zbl

[EGA III 2 1963] A. Grothendieck, "Eléments de géométrie algébrique, III: Étude cohomologique des faisceaux cohérents, II", Inst. Hautes Études Sci. Publ. Math. 17 (1963), 5-91. MR Zbl

[Friedman 1998] R. Friedman, Algebraic surfaces and holomorphic vector bundles, Springer, 1998. MR Zbl

[Geisser and Suzuki 2020] T. H. Geisser and T. Suzuki, "A Weil-étale version of the Birch and Swinnerton-Dyer formula over function fields", J. Number Theory 208 (2020), 367-389. MR Zbl

[Gold and Kisilevsky 1988] R. Gold and H. Kisilevsky, "On geometric $\mathbb{Z}_{p}$-extensions of function fields”, Manuscripta Math. 62:2 (1988), 145-161. MR Zbl

[González-Avilés and Tan 2007] C. D. González-Avilés and K.-S. Tan, "A generalization of the Cassels-Tate dual exact sequence", Math. Res. Lett. 14:2 (2007), 295-302. MR Zbl

[Greenberg 1999] R. Greenberg, "Iwasawa theory for elliptic curves", pp. 51-144 in Arithmetic theory of elliptic curves (Cetraro, Italy, 1997), edited by C. Viola, Lecture Notes in Math. 1716, Springer, 1999. MR Zbl

[Grothendieck 1968] A. Grothendieck, "Le groupe de Brauer, III: Exemples et compléments", pp. 88-188 in Dix exposés sur la cohomologie des schémas, Adv. Stud. Pure Math. 3, North-Holland, Amsterdam, 1968. MR Zbl

[Hartshorne 1977] R. Hartshorne, Algebraic geometry, Grad. Texts Math. 52, Springer, 1977. MR Zbl

[Illusie 1979] L. Illusie, "Complexe de de Rham-Witt et cohomologie cristalline”, Ann. Sci. École Norm. Sup. (4) 12:4 (1979), 501-661. MR Zbl

[Illusie 1983] L. Illusie, "Finiteness, duality, and Künneth theorems in the cohomology of the de Rham-Witt complex", pp. 20-72 in Algebraic geometry (Tokyo/Kyoto, 1982), edited by M. Raynaud and T. Shioda, Lecture Notes in Math. 1016, Springer, 1983. MR Zbl

[Illusie and Raynaud 1983] L. Illusie and M. Raynaud, "Les suites spectrales associées au complexe de de Rham-Witt", Inst. Hautes Études Sci. Publ. Math. 57 (1983), 73-212. MR Zbl

[Iwasawa 1973] K. Iwasawa, “On the $\mu$-invariants of $Z_{\ell}$-extensions”, pp. 1-11 in Number theory, algebraic geometry and commutative algebra, edited by Y. Kusunoki et al., Kinokuniya, Tokyo, 1973. MR Zbl

[Joshi 2020] K. Joshi, "Crystalline aspects of geography of low dimensional varieties, I: Numerology”, Eur. J. Math. 6:4 (2020), 1111-1175. MR

[Kahn 2014] B. Kahn, "A motivic formula for the $L$-function of an abelian variety over a function field", preprint, 2014. arXiv

[Kahn 2018a] B. Kahn, Fonctions zêta et L de variétés et de motifs, Calvage et Mounet, Paris, 2018. MR Zbl

[Kahn 2018b] B. Kahn, "Motifs et adjoints", Rend. Semin. Mat. Univ. Padova 139 (2018), 77-128. MR Zbl

[Kas 1977] A. Kas, "Weierstrass normal forms and invariants of elliptic surfaces", Trans. Amer. Math. Soc. 225 (1977), 259-266. MR Zbl

[Kato and Trihan 2003] K. Kato and F. Trihan, "On the conjectures of Birch and Swinnerton-Dyer in characteristic $p>0$ ", Invent. Math. 153:3 (2003), 537-592. MR Zbl 
[Kedlaya 2006] K. S. Kedlaya, "Fourier transforms and p-adic 'Weil II”, Compos. Math. 142:6 (2006), 1426-1450. MR Zbl

[Kleiman 2005] S. L. Kleiman, "The Picard scheme”, pp. 235-321 in Fundamental algebraic geometry (Trieste, Italy, 2003), Math. Surv. Monogr. 123, Amer. Math. Soc., Providence, RI, 2005. MR Zbl

[Lai et al. 2016] K. F. Lai, I. Longhi, K.-S. Tan, and F. Trihan, "The Iwasawa main conjecture for semistable abelian varieties over function fields", Math. Z. 282:1-2 (2016), 485-510. MR Zbl

[Li and Zhao 1997] C. Li and J. Zhao, "Iwasawa theory of $\mathbb{Z}_{p}^{d}$-extensions over global function fields", Exposition. Math. 15:4 (1997), 315-337. MR Zbl

[Liedtke 2013] C. Liedtke, "Algebraic surfaces in positive characteristic", pp. 229-292 in Birational geometry, rational curves, and arithmetic, edited by F. Bogomolov et al., Springer, 2013. MR Zbl

[Liu 2002] Q. Liu, Algebraic geometry and arithmetic curves, Oxford Grad. Texts Math. 6, Oxford Univ. Press, 2002. MR Zbl

[Liu et al. 2004] Q. Liu, D. Lorenzini, and M. Raynaud, "Néron models, Lie algebras, and reduction of curves of genus one", Invent. Math. 157:3 (2004), 455-518. Correction in 214:1 (2018), 593-604. MR Zbl

[Mazur 1972] B. Mazur, "Rational points of abelian varieties with values in towers of number fields", Invent. Math. 18 (1972), 183-266. MR Zbl

[Milne 1975] J. S. Milne, "On a conjecture of Artin and Tate", Ann. of Math. (2) 102:3 (1975), 517-533. MR Zbl

[Milne 1976] J. S. Milne, "Duality in the flat cohomology of a surface”, Ann. Sci. École Norm. Sup. (4) 9:2 (1976), 171-201. MR Zbl

[Milne 1980] J. S. Milne, Étale cohomology, Princeton Math. Ser. 33, Princeton Univ. Press, 1980. MR Zbl

[Milne 1986] J. S. Milne, Arithmetic duality theorems, Perspect. Math. 1, Academic, Boston, 1986. MR Zbl

[Milne and Ramachandran 2015] J. S. Milne and N. Ramachandran, "The $p$-cohomology of algebraic varieties and special values of zeta functions", J. Inst. Math. Jussieu 14:4 (2015), 801-835. MR Zbl

[Miranda 1981] R. Miranda, "The moduli of Weierstrass fibrations over $\mathbb{P}^{1}$ ", Math. Ann. 255:3 (1981), 379-394. MR Zbl

[Ochiai and Trihan 2009] T. Ochiai and F. Trihan, "On the Selmer groups of abelian varieties over function fields of characteristic p > 0", Math. Proc. Cambridge Philos. Soc. 146:1 (2009), 23-43. MR Zbl

[Pesenti and Szpiro 2000] J. Pesenti and L. Szpiro, "Inégalité du discriminant pour les pinceaux elliptiques à réductions quelconques", Compos. Math. 120:1 (2000), 83-117. MR Zbl

[Raynaud 1966] M. Raynaud, "Caractéristique d'Euler-Poincaré d'un faisceau et cohomologie des variétés abéliennes”, exposé 286 in Séminaire Bourbaki, 1964/1965, W. A. Benjamin, New York, 1966. Reprinted as pp. 129-147 in Séminaire Bourbaki 9, Soc. Math. France, Paris, 1995. MR Zbl

[Rosen 1973] M. Rosen, "The asymptotic behavior of the class group of a function field over a finite field", Arch. Math. (Basel) 24 (1973), 287-296. MR Zbl

[Schneider 1982] P. Schneider, "Zur Vermutung von Birch und Swinnerton-Dyer über globalen Funktionenkörpern”, Math. Ann. 260:4 (1982), 495-510. MR Zbl

[Seiler 1987] W. K. Seiler, "Global moduli for elliptic surfaces with a section”, Compos. Math. 62:2 (1987), 169-185. MR Zbl

[Serre 1966] J.-P. Serre, "Classes des corps cyclotomiques (d'après K. Iwasawa)", exposé 174 in Séminaire Bourbaki, 1958/1959, W. A. Benjamin, New York, 1966. Reprinted as pp. 83-93 in Séminaire Bourbaki 5, Soc. Math. France, Paris, 1995. MR

[Shimada 2020] I. Shimada, "The elliptic modular surface of level 4 and its reduction modulo 3", Ann. Mat. Pura Appl. (4) 199:4 (2020), 1457-1489. MR Zbl

[Shioda 1972] T. Shioda, "On elliptic modular surfaces”, J. Math. Soc. Japan 24 (1972), 20-59. MR Zbl

[Shioda 1975] T. Shioda, "Algebraic cycles on certain K3 surfaces in characteristic p", pp. 357-364 in Manifolds (Tokyo, 1973), edited by A. Hattori, 1975. MR Zbl

[Shioda 1986] T. Shioda, "An explicit algorithm for computing the Picard number of certain algebraic surfaces", Amer. J. Math. 108:2 (1986), 415-432. MR Zbl

[Shioda 1992] T. Shioda, "Some remarks on elliptic curves over function fields", pp. 99-114 in Journées arithmétiques (Geneva, 1991), edited by D. F. Coray and Y.-F. S. Pétermann, Astérisque 209, Soc. Math. France, Paris, 1992. MR Zbl 
[Silverman 1986] J. H. Silverman, The arithmetic of elliptic curves, Grad. Texts Math. 106, Springer, 1986. MR Zbl

[Sujatha 2010] R. Sujatha, "Elliptic curves and Iwasawa's $\mu=0$ conjecture", pp. 125-135 in Quadratic forms, linear algebraic groups, and cohomology, edited by J.-L. Colliot-Thélène et al., Dev. Math. 18, Springer, 2010. MR Zbl

[Sujatha 2011] S. Ramdorai, "On the $\mu$-invariant in Iwasawa theory", pp. 265-276 in WIN: women in numbers, edited by A.-C. Cojocaru et al., Fields Inst. Commun. 60, Amer. Math. Soc., Providence, RI, 2011. MR Zbl

[Suwa 1983] N. Suwa, "de Rham cohomology of algebraic surfaces with $q=-p_{a}$ in char. p", pp. 73-85 in Algebraic geometry (Tokyo/Kyoto 1982), edited by M. Raynaud and T. Shioda, Lecture Notes in Math. 1016, Springer, 1983. MR Zbl

[Suzuki 2020] T. Suzuki, "Duality for cohomology of curves with coefficients in abelian varieties", Nagoya Math. J. 240 (2020), 42-149. MR Zbl

[Tan 1995] K.-S. Tan, "Refined theorems of the Birch and Swinnerton-Dyer type", Ann. Inst. Fourier (Grenoble) 45:2 (1995), 317-374. MR Zbl

[Tan 2010] K.-S. Tan, “A generalized Mazur's theorem and its applications”, Trans. Amer. Math. Soc. 362:8 (2010), 4433-4450. MR Zbl

[Tan 2014] K.-S. Tan, “Selmer groups over $\mathbb{Z}_{p}^{d}$-extensions”, Math. Ann. 359:3-4 (2014), 1025-1075. MR Zbl

[Thakur 1994] D. S. Thakur, "Iwasawa theory and cyclotomic function fields", pp. 157-165 in Arithmetic geometry (Tempe, AZ, 1993), edited by N. Childress and J. W. Jones, Contemp. Math. 174, Amer. Math. Soc., Providence, RI, 1994. MR Zbl

[Trihan and Yasuda 2014] F. Trihan and S. Yasuda, "The $\ell$-parity conjecture for abelian varieties over function fields of characteristic $p>0$ ", Compos. Math. 150:4 (2014), 507-522. MR Zbl

[Ulmer 2002] D. Ulmer, "Elliptic curves with large rank over function fields", Ann. of Math. (2) 155:1 (2002), 295-315. MR $\mathrm{Zbl}$

[Ulmer 2011] D. Ulmer, "Elliptic curves over function fields", pp. 211-280 in Arithmetic of L-functions, edited by C. Popescu et al., IAS/Park City Math. Ser. 18, Amer. Math. Soc., Providence, RI, 2011. MR Zbl

[Ulmer 2019] D. Ulmer, "On the Brauer-Siegel ratio for abelian varieties over function fields", Algebra Number Theory 13:5 (2019), 1069-1120. MR Zbl

[Yuan 2018] X. Yuan, "Positivity of Hodge bundles of abelian varieties over some function fields", preprint, 2018. arXiv

[Zink 1984] T. Zink, Cartiertheorie kommutativer formaler Gruppen, Teubner-Texte Math. 68, Teubner, Leipzig, 1984. MR Zbl

Communicated by Joseph H. Silverman

Received 2019-10-03 Revised 2020-08-02 Accepted 2020-10-12

kinglaihonkon@163.com

ignaziol@iisc.ac.in

tsuzuki@gug.math.chuo-u.ac.jp

$\tan @ m a t h . n t u . e d u . t w$

f-trihan-52m@sophia.ac.jp
School of Mathematics and Statistics, Henan University, Henan, China

Department of Mathematics, Indian Institute of Science, Bangalore, India

Department of Mathematics, Chuo University, Tokyo, Japan

Department of Mathematics, National Taiwan University, Taipei, Taiwan

Department of Information and Communication Sciences, Sophia University, Tokyo, Japan 


\title{
Algebra \& Number Theory
}

\author{
msp.org/ant
}

\section{EDITORS}

MANAGING EdITOR

Bjorn Poonen

Massachusetts Institute of Technology

Cambridge, USA

\author{
EDITORIAL BOARD CHAIR \\ David Eisenbud \\ University of California \\ Berkeley, USA
}

BOARD OF EDITORS

Jason P. Bell

Bhargav Bhatt

Richard E. Borcherds

Frank Calegari

Antoine Chambert-Loir

J-L. Colliot-Thélène

Brian D. Conrad

Samit Dasgupta

Hélène Esnault

Gavril Farkas

Sergey Fomin

Edward Frenkel

Wee Teck Gan

Andrew Granville

Ben J. Green

Joseph Gubeladze

Christopher Hacon

Roger Heath-Brown

János Kollár
University of Waterloo, Canada

University of Michigan, USA

University of California, Berkeley, USA

University of Chicago, USA

Université Paris-Diderot, France

CNRS, Université Paris-Sud, France

Stanford University, USA

Duke University, USA

Freie Universität Berlin, Germany

Humboldt Universität zu Berlin, Germany

University of Michigan, USA

University of California, Berkeley, USA

National University of Singapore

Université de Montréal, Canada

University of Oxford, UK

San Francisco State University, USA

University of Utah, USA

Oxford University, UK

Princeton University, USA
Michael J. Larsen

Philippe Michel

Martin Olsson

Raman Parimala

Jonathan Pila

Irena Peeva

Anand Pillay

Michael Rapoport

Victor Reiner

Peter Sarnak

Michael Singer

Vasudevan Srinivas

Shunsuke Takagi

Pham Huu Tiep

Ravi Vakil

Michel van den Bergh

Akshay Venkatesh

Melanie Matchett Wood

Shou-Wu Zhang
Indiana University Bloomington, USA

École Polytechnique Fédérale de Lausanne

University of California, Berkeley, USA

Emory University, USA

University of Oxford, UK

Cornell University, USA

University of Notre Dame, USA

Universität Bonn, Germany

University of Minnesota, USA

Princeton University, USA

North Carolina State University, USA

Tata Inst. of Fund. Research, India

University of Tokyo, Japan

University of Arizona, USA

Stanford University, USA

Hasselt University, Belgium

Institute for Advanced Study, USA

Harvard University, USA

Princeton University, USA

\section{PRODUCTION}

production@msp.org

Silvio Levy, Scientific Editor

See inside back cover or msp.org/ant for submission instructions.

The subscription price for 2021 is US \$415/year for the electronic version, and \$620/year (+\$60, if shipping outside the US) for print and electronic. Subscriptions, requests for back issues and changes of subscriber address should be sent to MSP.

Algebra \& Number Theory (ISSN 1944-7833 electronic, 1937-0652 printed) at Mathematical Sciences Publishers, 798 Evans Hall \#3840, c/o University of California, Berkeley, CA $94720-3840$ is published continuously online. Periodical rate postage paid at Berkeley, CA 94704 , and additional mailing offices.

ANT peer review and production are managed by EditFLOW ${ }^{\circledR}$ from MSP.

PUBLISHED BY

mathematical sciences publishers

nonprofit scientific publishing

http://msp.org/

(C) 2021 Mathematical Sciences Publishers 


\section{Algebra \& Number Theory}

\section{Volume $15 \quad$ No. $4 \quad 2021$}

The Navarro conjecture for the alternating groups

OLIVIER BRUNAT and RISHI NATH

On the $\mu$-invariants of abelian varieties over function fields of positive characteristic

King-Fai LaI, Ignazio Longhi, TAKashi Suzuki, Ki-SEng TAN and Fabien Trihan

Tate modules of isocrystals and good reduction of Drinfeld modules

MAX (MAXIM) MORNEV

A proof of the Brown-Goodearl conjecture for module-finite weak Hopf algebras

DANIEL ROGALSKI, ROBERT WON and JAMES J. ZHANG

Chebyshev's bias in dihedral and generalized quaternion Galois groups

ALEXANDRE BAILLEUL

Integral $p$-adic Hodge theory of formal schemes in low ramification 